\title{
Introduction to the Langlands Program
}

\author{
A. W. Knapp
}

This article is an introduction to automorphic forms on the adeles of a linear reductive group over a number field. The first half is a summary of aspects of local and global class field theory, with emphasis on the local Weil group, the $L$ functions of Artin and Hecke, and the role of Artin reciprocity in relating the two kinds of $L$ functions. The first half serves as background for the second half, which discusses some structure theory for reductive groups, the definitions of automorphic and cusp forms, the Langlands $L$ group, $L$ functions, functoriality, and some conjectures. Much of the material in the second half may be regarded as a brief introduction to the Langlands program. There are ten sections:

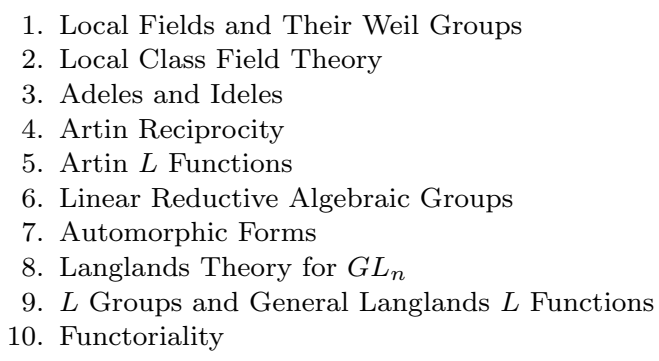

\section{Local Fields and Their Weil Groups}

This section contains a summary of information about local fields and their Weil groups. Four general references for this material are [Frö], [La], [Ta3], and [We4].

By a local field is meant any nondiscrete locally compact topological field. Let $F$ be a local field. If $\alpha$ is a nonzero element of $F$, then multiplication by $\alpha$ is an automorphism of the additive group of $F$ and hence carries additive Haar measure to a multiple of itself. This multiple is denoted $|\alpha|_{F}$, and it satisfies

$$
\int_{F} h\left(\alpha^{-1} x\right) d x=\int_{F} h(x) d(\alpha x)=|\alpha|_{F} \int_{F} h(x) d x,
$$

1991 Mathematics Subject Classification. Primary 11F70, 11R39, 11S37, 22 E55.

This article is based partly on lectures by Don Blasius in Edinburgh. The author is grateful to Jonathan Rogawski and David Vogan for offering a number of suggestions about the exposition, to Dinakar Ramakrishnan for supplying a proof of Theorem 8.8 and giving permission to include it here, and to Hervé Jacquet for answering many questions. 
where $d x$ is an additive Haar measure. We refer to $|\alpha|_{F}$ as the module of $\alpha$. By convention, $|0|_{F}=0$. The function $\alpha \mapsto|\alpha|_{F}$ is continuous on $F$ and is a homomorphism of the multiplicative group $F^{\times}$into the multiplicative group $\mathbb{R}_{+}^{\times}$of positive reals.

The local field $F$ is said to be nonarchimedean if $|\alpha|_{F}$ satisfies the ultrametric inequality

Otherwise $F$ is archimedean.

$$
|\alpha+\beta| \leq \max \{|\alpha|,|\beta|\}
$$

A classification of local fields appears in [We4]. There are only two archimedean local fields, $\mathbb{R}$ and $\mathbb{C}$. In the nonarchimedean case, the set of nonzero values of $|\cdot|_{F}$ is a discrete subgroup of $\mathbb{R}_{+}^{\times}$. The nonarchimedean local fields divide into two kinds. Those of characteristic 0 turn out to be the $p$-adic fields, namely the finite extensions of the fields $\mathbb{Q}_{p}$ of $p$-adic numbers for each prime number $p$. Those of characteristic not 0 turn out to be the fields of Laurent series in one variable (finite in negative powers) over the various finite fields. In this article we consider only local fields of characteristic 0 .

Let us review various constructions of $\mathbb{Q}_{p}$. One way to define $\mathbb{Q}_{p}$ is as the completion of $\mathbb{Q}$ in the metric $d(x, y)=|x-y|_{p}$, where $\left|a p^{n} / b\right|_{p}=p^{-n}$ if $a$ and $b$ are integers prime to $p$. The metric extends to $\mathbb{Q}_{p}$, giving it a locally compact topology, and the field operations extend as well. It is easy to see that $|\alpha|_{\mathbb{Q}_{p}}$, which we abbreviate as $|\alpha|_{p}$, is just the distance between $\alpha$ and 0 in the completed metric.

Another construction begins with the definition of the maximal compact ring $\mathbb{Z}_{p}$ of $p$-adic integers, which is taken to be the inverse limit

$$
\mathbb{Z}_{p}=\underset{\leftarrow}{\lim _{n}} \mathbb{Z} /\left(p^{n}\right)
$$

The ring $\mathbb{Z}_{p}$ is an integral domain having a unique maximal ideal, namely $p \mathbb{Z}_{p}$. The ideals $p^{n} \mathbb{Z}_{p}$ form a neighborhood basis of 0 , and $\left\{\alpha+p^{n} \mathbb{Z}_{p}\right\}$ is a neighborhood basis about $\alpha \in \mathbb{Z}_{p}$. Then $\mathbb{Q}_{p}$ may be described algebraically as the field of quotients of $\mathbb{Z}_{p}$ or as $\mathbb{Z}_{p} \otimes \mathbb{Q}$ or as $\mathbb{Z}\left[p^{-1}\right]=\bigcup_{n \geq 1} p^{-n} \mathbb{Z}_{p}$. The last of these descriptions provides the topology; each $p^{-n} \mathbb{Z}_{p}$ is to be open and homeomorphic with $\mathbb{Z}_{p}$. The inclusion $\mathbb{Z}_{p} \subset \mathbb{Q}_{p}$ makes $\mathbb{Z}_{p}$ a compact subring of the local field $\mathbb{Q}_{p}$. Here $\mathbb{Z}_{p}$ is precisely the set of all $\alpha \in \mathbb{Q}_{p}$ with $|\alpha|_{p} \leq 1$, and the maximal ideal $p \mathbb{Z}_{p}$ is the set of all $\alpha \in \mathbb{Q}_{p}$ with $|\alpha|_{p}<1$.

Let $K$ be a finite extension of $\mathbb{Q}_{p}$ with $\left[K: \mathbb{Q}_{p}\right]=n$. If $K$ is decomposed as a direct sum of $n$ one-dimensional $\mathbb{Q}_{p}$ vector spaces, then the decomposition automatically respects the topology. The set

$$
\mathcal{O}_{K}=\left\{\left.\alpha \in K|| \alpha\right|_{K} \leq 1\right\}
$$

is a compact open subring of $K$ called the ring of integers of $K$. It is the unique maximal compact subring of $K$ and is equal to the integral closure of $\mathbb{Z}_{p}$ in $K$. The group of units $\mathcal{O}_{K}^{\times}$of $\mathcal{O}_{K}$ is the set of elements $\alpha$ with $|\alpha|_{K}=1$. The ring $\mathcal{O}_{K}$ has the following properties:

1) $\mathcal{O}_{K}$ has a unique maximal ideal $\mathfrak{m}_{K}$, namely the set of elements $\alpha$ with $|\alpha|_{K}<1$. The ideal $\mathfrak{m}_{K}$ is principal, having any element of maximal module as generator. Such an element will typically be denoted $\varpi_{K}$ and is a prime element. Every nonzero ideal of $\mathcal{O}_{K}$ is principal and is a power of $\mathfrak{m}_{K}$ (with the corresponding power of $\varpi_{K}$ as generator).

2) $\mathcal{O}_{K}$ is the inverse limit of $\mathcal{O}_{K} /\left(\varpi_{K}^{n}\right)$ on $n$. 
3) $k_{K}=\mathcal{O}_{K} / \mathfrak{m}_{K}$ is a finite field $k_{K}$, the residue field. The inclusion of $\mathbb{Z}_{p}$ into $\mathcal{O}_{K}$ induces a map of $\mathbb{Z}_{p} / p \mathbb{Z}_{p}$ into $k_{K}$, and thus $\mathbb{Z}_{p} / p \mathbb{Z}_{p}$ may be regarded as the prime field of $k_{K}$. We write the number of elements in $k_{K}$ as $q=p^{f}$ and call $f$ the residue degree of $K$ over $\mathbb{Q}_{p}$. The module of $\varpi_{K}$ is $q^{-1}$.

4) By (1), the ideal $p \mathcal{O}_{K}$ of $\mathcal{O}_{K}$ is of the form $\mathfrak{m}_{K}^{e}=\left(\varpi_{K}^{e}\right)$ for some integer $e$; $e$ is called the ramification degree of $K$ over $\mathbb{Q}_{p}$.

5) $\left[K: \mathbb{Q}_{p}\right]=e f$ since $p^{-n}=|p|_{K}=\left|\varpi_{K}\right|_{K}^{e}=q^{-e}$.

Now let $K \supset F$ be two finite extensions of $\mathbb{Q}_{p}$, and write $q_{K}, f_{K}, e_{K}$ and $q_{F}, f_{F}, e_{F}$ for the respective integers $q, f, e$. Also let $k_{K}$ and $k_{F}$ be the residue fields. Then we have an inclusion $k_{F} \subset k_{K}$. We define $f=\left[k_{K}: k_{F}\right]$, and we let $e$ be the integer such that $\varpi_{F} \mathcal{O}_{K}=\mathfrak{m}_{K}^{e}$. The integers $f$ and $e$ are the residue degree and ramification degree of $K$ over $F$. Then

$$
f=f_{K} / f_{F}, \quad e=e_{K} / e_{F}, \quad \text { and } \quad[K: F]=e f .
$$

Fix finite extensions $K \supset F$ of $\mathbb{Q}_{p}$, and suppose that $K / F$ is Galois. Any $F$ automorphism of $K$ is module-preserving and hence maps $\mathcal{O}_{K}$ to itself and $\mathfrak{m}_{K}$ to itself. It therefore induces a automorphism of the quotient, which is the residue field $k_{K}$, and this automorphism fixes the residue field $k_{F}$ of $F$. The result is a homomorphism $\operatorname{Gal}(K / F) \rightarrow \operatorname{Gal}\left(k_{K} / k_{F}\right)$. Since $\operatorname{Gal}\left(k_{K} / k_{F}\right)$ is cyclic with generator $x \mapsto x^{q_{F}}$, the image of a member of $\operatorname{Gal}(K / F)$ is necessarily of the form $x \mapsto x^{q_{F}^{n}}$ for some integer $n$.

Theorem 1.1. If $K / F$ is a Galois extension of finite extensions of $\mathbb{Q}_{p}$ and if $k_{K}$ and $k_{F}$ are the respective residue fields, then $\operatorname{Gal}(K / F)$ maps onto $\operatorname{Gal}\left(k_{K} / k_{F}\right)$.

REFEREnCE. See [La, p. 15]. For a formulation of this result without the assumption that $K / F$ is Galois, see [Frö, p. 26].

In the setting of Theorem 1.1, we obtain an exact sequence

$$
1 \longrightarrow I_{K / F} \longrightarrow \operatorname{Gal}(K / F) \longrightarrow \operatorname{Gal}\left(k_{K} / k_{F}\right) \longrightarrow 0,
$$

where $I_{K / F}$ is the kernel, which is called the inertia group of $K$ over $F$. We shall be interested in the effect on (1.1) of letting $K$ swell to $\bar{F}$.

For the moment let us drop the assumption that $K / F$ is Galois. Let $f$ and $e$ be the residue and ramification degrees of $K$ over $F$. We say that the extension $K / F$ is unramified if $e=1$. In the Galois case, the group $\operatorname{Gal}\left(k_{K} / k_{F}\right)$ has order $f$, and the exactness of (1.1) implies that $I_{K / F}$ has order $e$; thus $K / F$ is unramified if and only if $\operatorname{Gal}(K / F) \rightarrow \operatorname{Gal}\left(k_{K} / k_{F}\right)$ is an isomorphism.

Theorem 1.2. Let $F$ be a finite extension of $\mathbb{Q}_{p}$, and let $k$ be a finite extension of the residue field $k_{F}$. Then there exists an unramified finite extension $K$ of $F$ with residue field $k_{K} \cong k$. Such a field $K$ is unique up to $F$ isomorphism and is Galois over F.

Reference. [Frö, p. 26] or [Se2, p. 54].

Consequently for each $f \geq 1$, there is, up to $F$ isomorphism, a unique unramified extension $K=K_{f}$ of $F$ of degree $f$, and $K / F$ is Galois. By Theorem 1.1, $\operatorname{Gal}(K / F) \cong \operatorname{Gal}\left(k_{K} / k_{F}\right)$ is a cyclic group of order $q=p^{f}$ whose generator Fr is the lift of the generator $x \mapsto x^{\left|k_{F}\right|}$ of $\operatorname{Gal}\left(k_{K} / k_{F}\right)$. The element $\operatorname{Fr}$ of $\operatorname{Gal}(K / F)$ is 
called the Frobenius element and is characterized among members of $\mathrm{Gal}(K / F)$ by the congruence $\operatorname{Fr}(x) \equiv x^{\left|k_{F}\right|} \bmod \mathfrak{m}_{K}$.

Fix an algebraic closure $\bar{F}$ of $F$, and regard each $K=K_{f}$ as contained in $\bar{F}$. When two residue degrees $f$ and $f^{\prime}$ have the property that $f$ divides $f^{\prime}$, the multiplicative property of residue degrees and ramification degrees implies that $K_{f} \subset K_{f^{\prime}}$. The result, as $f$ varies, is a directed system of subfields of $\bar{F}$. The union of these subfields is called the maximal unramified extension of $F$ and is denoted $F_{\text {ur }}$.

Every field map over $F$ of a subfield of $\bar{F}$ into $\bar{F}$ extends to an automorphism of $\bar{F}$, and it follows that every member of $\operatorname{Gal}\left(F_{\mathrm{ur}} / F\right)$ extends to a member of $\operatorname{Gal}(\bar{F} / F)$. In other words, there is an exact sequence

$$
1 \longrightarrow I_{F} \longrightarrow \operatorname{Gal}(\bar{F} / F) \longrightarrow \operatorname{Gal}\left(F_{\text {ur }} / F\right) \longrightarrow 0
$$

in which $I_{F}$ is the kernel of $\pi$. The group $I_{F}$ is called the inertia group of $F$ (or of $\bar{F}$ over $F$ ).

Now let us take the inverse limit of (1.1), letting $K$ swell to $\bar{F}$. Then we obtain a homomorphism of $\operatorname{Gal}(\bar{F} / F)$ into $\operatorname{Gal}\left(\bar{k}_{F} / k_{F}\right)$, where $\bar{k}_{F}$ is the algebraic closure of $k_{F}$. Then we have the following result.

Theorem 1.3. Every finite extension of $F$ in $F_{\mathrm{ur}}$ is unramified. The natural homomorphism of $\operatorname{Gal}(\bar{F} / F)$ into $\operatorname{Gal}\left(\bar{k}_{F} / k_{F}\right)$ descends via (1.2) to a topological isomorphism of $\operatorname{Gal}\left(F_{\mathrm{ur}} / F\right)$ onto $\operatorname{Gal}\left(\bar{k}_{F} / k_{F}\right)$.

REFERENCE. [Frö, p. 28] or [Se2, p. 54-55].

Let us identify $\operatorname{Gal}\left(\bar{k}_{F} / k_{F}\right)$, so that we can make (1.2) more explicit. The field $\bar{k}_{F}$ is the union of its finite subfields. These form a directed system under divisibility of degree, and the degree can be any positive integer. Therefore $\operatorname{Gal}\left(\bar{k}_{F} / k_{F}\right)$ is the inverse limit of cyclic groups $\mathbb{Z} / n \mathbb{Z}$, indexed according to divisibility of the indices $n$. The resulting compact group is denoted $\widehat{\mathbb{Z}}$ and is isomorphic to $\prod_{p} \mathbb{Z}_{p}$. We regard the Frobenius element $x \mapsto x^{\left|k_{F}\right|}$ in $\operatorname{Gal}\left(\bar{k}_{F} / k_{F}\right)$ as the integer +1 , and the subgroup $\mathbb{Z}$ of $\widehat{\mathbb{Z}}$ generated by +1 is dense in $\widehat{\mathbb{Z}}$. The isomorphism of Theorem 1.3 tells us that $\operatorname{Gal}\left(F_{\text {ur }} / F\right) \cong \widehat{\mathbb{Z}}$, and we let Fr be the (Frobenius) element of $\operatorname{Gal}\left(F_{\text {ur }} / F\right)$ that corresponds to +1 in $\widehat{\mathbb{Z}}$. The rewritten form of $(1.2)$ is then

$$
1 \longrightarrow I_{F} \longrightarrow \operatorname{Gal}(\bar{F} / F) \stackrel{\pi}{\longrightarrow} \widehat{\mathbb{Z}} \longrightarrow 0
$$

where $\pi$ is defined to be restriction from $\bar{F}$ to $F_{\text {ur }}$ composed with the isomorphism to $\widehat{\mathbb{Z}}$.

The Langlands theory of $L$ groups and $L$ functions makes extensive use of the Weil group of a local field. Let us define this group, sketch some of its properties, and formulate the theorems of local class field theory in terms of it. A reference for Weil groups is [Ta3].

We continue with $F$ as a finite extension of $\mathbb{Q}_{p}$ and with $\bar{F}$ as an algebraic closure of $F$; the archimedean case is postponed to the next section. Let $G_{F}=$ $\operatorname{Gal}(\bar{F} / F)$. We write $\widehat{\mathbb{Z}}=\operatorname{Gal}\left(F_{\text {ur }} / F\right)$, and we let $\mathbb{Z}$ be the infinite cyclic subgroup of $\widehat{\mathbb{Z}}$ generated by the Frobenius element Fr. With $\pi$ as in (1.3), the Weil group of $F$ is defined as an abstract group by $W_{F}=\pi^{-1}(\mathbb{Z}) \subset \operatorname{Gal}(\bar{F} / F)$. The Weil form 
of the exact sequence (1.3) is then

$$
1 \longrightarrow I_{F} \longrightarrow W_{F} \stackrel{\pi}{\longrightarrow} \mathbb{Z} \longrightarrow 0 .
$$

The relative topology from (1.3) gives $\mathbb{Z}$ an unusual topology, but we change matters to give $\mathbb{Z}$ the discrete topology. Correspondingly we retopologize $W_{F}$ so that $\pi$ is continuous and $I_{F}$ is homeomorphic with $\pi^{-1}(\{0\})$.

To understand $\pi$ and $W_{F}$ better, let $K / F$ be a finite Galois extension. Then $G_{F}$ maps onto $\operatorname{Gal}(K / F)$ with kernel $G_{K}$, and in turn $\operatorname{Gal}(K / F)$ maps onto $\operatorname{Gal}\left(k_{K} / k_{F}\right)$ by Theorem 1.1. The effect is to associate to any member $\sigma$ of $G_{F}$ an integer $n$ and the automorphism $x \mapsto x^{q_{F}^{n}}$, with $n$ depending on $K$ and defined modulo the residue degree of $K / F$. The inverse limit of the resulting tuple of $n$ 's, as $K$ varies, defines the member $\pi(\sigma)$ of $\widehat{Z}$. For $\pi(\sigma)$ to be in $\mathbb{Z}$, the condition is that the inverse limit can be regarded as a single integer $n$. That is, the members of the Weil group $W_{F}$ are those members of $G_{F}$ that induce on the algebraic closure $\bar{k}_{F}$ the automorphism $x \mapsto x^{q_{F}^{n}}$ for some integer $n$.

If $E / F$ is a finite extension, then Galois theory says that $G_{E}$ is the subgroup of $G_{F}$ fixing $E$. Hence $W_{E}$ is a subgroup of $G_{F}$. The next theorem identifies this subgroup.

Theorem 1.4. If $E / F$ is a finite extension, then $W_{E}=G_{E} \cap W_{F}$.

Proof. We may regard $k_{E}$ as containing $k_{F}$ with $\bar{k}_{E}=\bar{k}_{F}$. The subgroup $G_{E} \cap W_{F}$ consists of the members of $G_{F}$ that induce on $\bar{k}_{F}$ the automorphism $x \mapsto x^{q_{F}^{n}}$ for some integer $n$ and that fix $k_{E}$. Then $x^{q_{F}^{n}}=x$ for all $x \in k_{E}$, and it follows that $\left[k_{E}: k_{F}\right]$ divides $n$. If we let $a=n /\left[k_{E}: k_{F}\right]$, then $q_{F}^{n}=q_{F}^{\left[k_{E}: k_{F}\right] a}=q_{E}^{a}$. Hence $G_{E} \cap W_{F} \subset W_{E}$. The reverse inclusion is trivial, and the theorem follows.

Since $\mathbb{Z}$ is dense in $\widehat{\mathbb{Z}}$, it follows that $W_{F}$ is dense in $G_{F}$. If $E / F$ is a finite extension, then $G_{F} / G_{E}$ is a finite set, and the image of $W_{F}$ in it is dense. Therefore $W_{F}$ maps onto $G_{F} / G_{E}$. Because of Theorem 1.4, we obtain a bijection

$$
W_{F} / W_{E} \stackrel{\sim}{\rightarrow} G_{F} / G_{E} \cong \operatorname{Hom}_{F}(E, \bar{F}),
$$

where $\operatorname{Hom}_{F}(E, \bar{F})$ is the set of indicated field maps. If $E / F$ is Galois, then $\operatorname{Hom}_{F}(E, \bar{F})=\operatorname{Gal}(E / F)$ and (1.5) is a group isomorphism.

Let us mention an alternate definition of $W_{F}$. If $G$ is a topological group, we let $G^{c}$ be the closure of the commutator subgroup and we define $G^{\mathrm{ab}}=G / G^{c}$. The closed subgroup $G_{F}^{c}$ of $G_{F}=\operatorname{Gal}(\bar{F} / F)$ corresponds to a subfield $F^{\text {ab }}$ of $\bar{F}$ called the maximal abelian extension of $F$. Its Galois group $\operatorname{Gal}\left(F^{\mathrm{ab}} / F\right)$ is just $G_{F}^{\mathrm{ab}}$.

Let $K$ be a finite Galois extension of $F$ lying in $\bar{F}$, and form $K^{\mathrm{ab}}$. Since $\operatorname{Gal}\left(F_{\text {ur }} / F\right)=\widehat{\mathbb{Z}}$ is abelian, we have $K^{\mathrm{ab}} \supset F^{\mathrm{ab}} \supset F_{\text {ur }}$. Therefore we have surjective maps induced by restriction:

$$
\operatorname{Gal}(\bar{F} / F) \rightarrow \operatorname{Gal}\left(K^{\mathrm{ab}} / F\right) \rightarrow \operatorname{Gal}\left(F_{\text {ur }} / F\right)=\widehat{\mathbb{Z}}
$$

The inverse image of $\mathbb{Z} \subset \widehat{\mathbb{Z}}$ in $\operatorname{Gal}(\bar{F} / F)$ was defined to be $W_{F}$, and we define the intermediate inverse image of $\mathbb{Z}$ in $\operatorname{Gal}\left(K^{\mathrm{ab}} / F\right)$ to be $W_{K / F}$. This construction carries with it surjective maps $W_{F} \rightarrow W_{K / F}$, and these are compatible as $K$ varies. In addition, any element of $\bar{F}$ is in some finite Galois extension $K$ of $F$ and therefore 
is also in $K^{\text {ab }}$. Hence no nontrivial element of $W_{F}$ can restrict to the identity on every $W_{K / F}$, and it follows that $W_{F}$ is the inverse limit

$$
W_{F}=\lim _{K} W_{K / F}
$$

\section{Local Class Field Theory}

In this section we shall state the main results from local class field theory and translate them into statements about Weil groups. For most of this section, we let $F$ be a finite extension of $\mathbb{Q}_{p}$ and $\bar{F}$ be an algebraic closure of $F$. References for the material in this section are [Ne], [Se1], [Se2], and [Ta3].

Let $K$ be a finite Galois extension of $F$ lying in $\bar{F}$ and having $[K: F]=n$, and let $G_{K / F}=\operatorname{Gal}(K / F)$. It is known that $H^{2}\left(G_{K / F}, K^{\times}\right)$is cyclic of order $n$ with a canonical generator $u_{K / F}$. This can be proved rather quickly with the aid of the theory of the Brauer group [Se1, p. 137], and also a direct cohomological proof is possible [Se1, p. 130].

Theorem 2.1. If $K / F$ is a finite Galois extension, then the canonical generator $u_{K / F}$ of $H^{2}\left(G_{K / F}, K^{\times}\right)$defines (by means of "cup product") an isomorphism of $G_{K / F}^{\mathrm{ab}}$ onto $F^{\times} / N_{K / F}\left(K^{\times}\right)$, where $N_{K / F}(\cdot)$ denotes the norm map.

REFERENCE. [Se1, p. 140].

The inverse $\theta_{K / F}: F^{\times} / N_{K / F}\left(K^{\times}\right) \rightarrow G_{K / F}^{\mathrm{ab}}$ of the isomorphism in Theorem 2.1 is called the local reciprocity map of $K / F$. When $G_{K / F}$ is abelian, then $G_{K / F}^{\mathrm{ab}}$ equals $G_{K / F}$ and $\theta_{K / F}$ is an isomorphism of $F^{\times} / N_{K / F}\left(K^{\times}\right)$onto $G_{K / F}$.

If $x \in F^{\times}$lies in the coset $\bar{x}$ of $F^{\times} / N_{K / F}\left(K^{\times}\right)$, then we write $\theta_{K / F}(\bar{x})=$ $(x, K / F)$.

The symbols $(x, K / F)$ define homomorphisms $F^{\times} \rightarrow G_{K / F}$ that are compatible [Se1, p. 140] when $K^{\prime} \supset K \supset F$ and $K^{\prime} / F$ is finite abelian. Taking the inverse limit, we obtain a homomorphism $\theta_{F}: F^{\times} \rightarrow G_{F^{\mathrm{ab}} / F}$ or $\theta_{F}: F^{\times} \rightarrow G_{F}^{\mathrm{ab}}$.

In the exact sequence (1.3), the homomorphism $\pi$ has an abelian image and therefore descends to a homomorphism $\pi^{\mathrm{ab}}$ defined on $G_{F}^{\mathrm{ab}}=G_{F^{\mathrm{ab}} / F}$. Accordingly we replace (1.3) by

$$
1 \longrightarrow I_{F^{\mathrm{ab}} / F} \longrightarrow G_{F}^{\mathrm{ab}} \stackrel{\pi^{\mathrm{ab}}}{\longrightarrow} \widehat{\mathbb{Z}} \longrightarrow 0,
$$

where $\widehat{\mathbb{Z}}=G_{F_{\text {ur }} / F}$ and where $I_{F^{\mathrm{ab}} / F}$ is the kernel of $\pi^{\mathrm{ab}}$. By Galois theory we may interpret $I_{F^{\mathrm{ab}} / F}$ as $G_{F^{\mathrm{ab}} / F_{\mathrm{ur}}}$.

We shall compute $\pi^{\mathrm{ab}} \circ \theta_{F}: F^{\times} \rightarrow \widehat{\mathbb{Z}}$. For any $x \in F^{\times}$, the number $|x|_{F}$ is a power of $q^{-1}$, and we define $v(x)$ to be that power.

Theorem 2.2. If $K / F$ is a finite unramified extension and if $\operatorname{Fr}$ in $G_{K / F}$ denotes the Frobenius element, then $(x, K / F)=\mathrm{Fr}^{v(x)}$ for all $x \in F^{\times}$.

RefEREnce. [Se1, p. 141].

Corollary 2.3. For any $x \in F^{\times}, \pi^{\mathrm{ab}}\left(\theta_{F}(x)\right)=v(x)$ as a member of $\widehat{\mathbb{Z}}$.

Any inverse image in $G_{F}$ of the element $\theta_{F}(x)$ of $G_{F}^{\text {ab }}$ therefore lies in the Weil group $W_{F}$. 
We shall prove below in Lemma 2 that the homomorphism $W_{F}^{\mathrm{ab}} \rightarrow G_{F}^{\mathrm{ab}}$ induced by $W_{F} \rightarrow G_{F}$ is one-one, and then we may regard $\left(\pi^{\mathrm{ab}}\right)^{-1}(\mathbb{Z})$ as $W_{F}^{\mathrm{ab}}$. Thus (2.1) gives us an exact sequence

$$
1 \longrightarrow G_{F^{\mathrm{ab}} / F_{\mathrm{ur}}} \longrightarrow W_{F}^{\mathrm{ab}} \stackrel{\pi^{\mathrm{ab}}}{\longrightarrow} \mathbb{Z} \longrightarrow 0 .
$$

Lemma 1. $W_{F}^{c}=G_{F}^{c}$.

Proof. Certainly $W_{F}^{c} \subset G_{F}^{c}$, and it is $G_{F}^{c} \subset W_{F}^{c}$ that needs proof. Let $x$ be a member of $W_{F}$ with $\pi(x)=1 \in \mathbb{Z}$. Then $W_{F}$ is the semidirect product of $\left\{x^{n}\right\}_{n=-\infty}^{\infty}$ with $I_{F}$. So $W_{F}^{c}$ is the closure of the subgroup of $I_{F}$ generated by all commutators of $I_{F}$ and all elements $x^{n} i x^{-n} i^{-1}$ with $i \in I_{F}$.

Let $Y$ be the smallest closed subgroup of $G_{F}$ containing $x$. Since $Y$ is compact abelian and $\mathbb{Z}$ is dense in $\widehat{\mathbb{Z}}, \pi(Y)=\widehat{\mathbb{Z}}$. If $g \in G_{F}$ is given, choose $y \in Y$ with $\pi(g)=\pi(y)$. Then $\pi\left(g y^{-1}\right)=0$ shows that $g y^{-1}$ is in $I_{F}$, and the identity $g=$ $\left(g y^{-1}\right) y$ therefore shows that every element of $G_{F}$ is the product of an element of $I_{F}$ and an element of $Y$. So $G_{F}^{c}$ is the closure of the subgroup of $I_{F}$ generated by all commutators of $I_{F}$ and all elements $y i y^{-1} i^{-1}$ with $y \in Y$ and $i \in I_{F}$. The commutators of $I_{F}$ are in $W_{F}^{c}$, and the element yiy ${ }^{-1} i^{-1}$ is the limit of elements $x^{n} i x^{-n} i^{-1}$ with $n$ varying through a suitable sequence. Hence $y i y^{-1} i^{-1}$ is in $W_{F}^{c}$, and $G_{F}^{c} \subset W_{F}^{c}$.

Lemma 2. The homomorphism $W_{F}^{\mathrm{ab}} \rightarrow G_{F}^{\mathrm{ab}}$ induced by $W_{F} \rightarrow G_{F}$ is one-one.

Proof. We need to prove that $W_{F} \cap G_{F}^{c}=W_{F}^{c}$. Since $G_{F}^{c} \subset I_{F} \subset W_{F}$, we need $G_{F}^{c}=W_{F}^{c}$. But this is just what Lemma 1 gives.

With Lemma 2 proved, (2.2) now follows. Corollary 2.3 implies that the restriction of $\theta_{F}$ to $\mathcal{O}_{F}^{\times}$is in the kernel of $\pi^{\mathrm{ab}}$ on $W_{F}^{\mathrm{ab}}$. Hence it is in the image of $G_{F}$ ab $/ F_{\mathrm{ur}}$. We can put this information and the full strength of Corollary 2.3 together in a diagram with exact rows and commutative squares

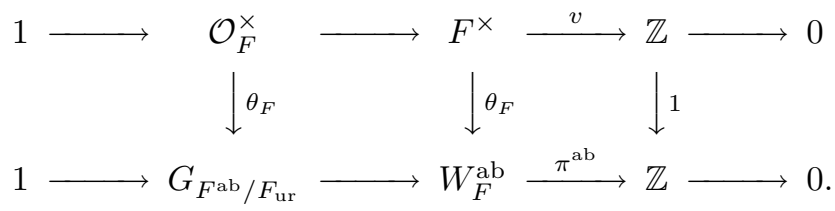

Let us now state in its classical form the Existence Theorem of local class field theory.

Theorem 2.4. The map $K \rightarrow F^{\times} / N_{K / F}\left(K^{\times}\right)$is a bijection of the set of finite abelian Galois extensions $K$ of $F$ onto the set of open subgroups of $F^{\times}$of finite index.

Reference. [Se1, p. 143].

Use of the Weil group allows us to restate this result more simply.

Corollary 2.5. The local reciprocity map $\theta_{F}$ is a topological isomorphism of $F^{\times}$onto $W_{F}^{\mathrm{ab}}$. 
Proof. If $K / F$ is a finite abelian extension within $F^{\mathrm{ab}}$, then Theorem 2.1 shows that the composition of $\theta_{F}$ followed by the quotient map $G_{F}^{\mathrm{ab}} \rightarrow G_{K / F}$ carries $F^{\times}$ onto $G_{K / F}$. Letting $K$ vary, we see that $\theta_{F}$ carries $F^{\times}$onto a dense subgroup of $G^{\mathrm{ab}}$. Since $\mathcal{O}_{F}^{\times}$is compact, it follows from $(2.3)$ that $\theta_{F}$ carries $\mathcal{O}_{F}^{\times}$onto $G_{F \mathrm{ab}} / F_{\mathrm{ur}}$. A second application of (2.3) shows that $\theta_{F}$ carries $F^{\times}$onto $W_{F}^{\text {ab }}$. The kernel of $\theta_{F}$ is $\bigcap_{K} N_{K / F}\left(K^{\times}\right)$, the intersection being taken over all finite extensions $K$ of $F$ lying in $F^{\mathrm{ab}}$. If $i$ and $j$ are any integers $\geq 0$, then the set $\left\{\left(\varpi_{F}^{i}\right)^{n}\left(1+\mathcal{O}_{F}^{j}\right) \mid n \in \mathbb{Z}\right\}$ is an open subgroup of $F^{\times}$of finite index. By Theorem 2.4 it is $N_{K / F}\left(K^{\times}\right)$for some finite abelian $K / F$. Hence

$$
\bigcap_{K} N_{K / F}\left(K^{\times}\right) \subset \bigcap_{i, j}\left\{\left(\varpi_{F}^{i}\right)^{n}\left(1+\mathcal{O}_{F}^{j}\right) \mid n \in \mathbb{Z}\right\}=\{1\},
$$

and $\theta_{F}$ is one-one. Consequently $\theta_{F}: F^{\times} \rightarrow W_{F}^{\text {ab }}$ is a group isomorphism. Since $\theta_{F}: \mathcal{O}_{F}^{\times} \rightarrow G_{F_{\mathrm{ab}} / F_{\mathrm{ur}}}$ is continuous and $\mathcal{O}_{F}^{\times}$is compact, $\theta_{F}: \mathcal{O}_{F}^{\times} \rightarrow G_{F \text { ab } / F_{\text {ur }}}$ is a homeomorphism. Then it follows that $\theta_{F}: F^{\times} \rightarrow W_{F}^{\text {ab }}$ is a homeomorphism.

\section{REMARKS.}

1) In Corollary $2.5, \theta_{F}$ carries a prime element of $\mathcal{O}_{F}$ to an element of $W_{F}^{\text {ab }}$ that acts as a Frobenius automorphism in every unramified extension. Thus $\theta_{F}(x)(y)=$ $y^{|x|_{F}^{-1}}$ for $y$ in any unramified extension. Some authors adjust a sign somewhere to make $\theta_{F}(x)(y)=y^{|x|_{F}}$; see [Ta3, p. 6] for a discussion of this point. For these authors the later definitions of $L$ functions are likely to be what we, with our traditional definitions, would call the $L$ function of the contragredient.

2) Theorem 2.4, which is the difficult result in local class field theory, is essentially equivalent with Corollary 2.5. A proof that Corollary 2.5 implies Theorem 2.4 may be based on [Se1, p. 144].

3) Corollary 2.5 implies that the (continuous) one-dimensional representations of $F^{\times}$are parametrized by the continuous homomorphisms of $W_{F}$ into $\mathbb{C}^{\times}$. This is a point of departure for conjectures of Langlands about parametrizing irreducible representations of linear reductive groups over $F$. We return to this matter in $\S 8$.

Let us return to the group $W_{K / F}$ defined from (1.6), where $K$ is a finite Galois extension of $F$ lying in $\bar{F}$. The kernel of the map $W_{F} \rightarrow W_{K / F}$ in (1.7), in view of (1.6), is the set of all $w \in W_{F}$ that act as Galois elements by 1 on $K^{\text {ab }}$. They are in particular members of $G_{F}$. Being 1 on $K$, they are in $G_{K}$. Being 1 on $K^{\mathrm{ab}}$, they are in $G_{K}^{c}$, which equals $W_{K}^{c}$ by Lemma 1 . As a result we have

$$
W_{K / F} \cong W_{F} / W_{K}^{c} \text {. }
$$

We form the exact sequence

$$
1 \longrightarrow W_{K} / W_{K}^{c} \longrightarrow W_{F} / W_{K}^{c} \longrightarrow W_{F} / W_{K} \longrightarrow 0 .
$$

The quotient $W_{K} / W_{K}^{c}$ is just $W_{K / K}$ by (2.4), and this is by definition the subgroup of $G_{K}^{\mathrm{ab}}$ inducing an integral power of the Frobenius. By Lemma 2 and the derivation of $(2.2)$, we can identify this subgroup with $W_{K}^{\mathrm{ab}}$. Applying Corollary 2.5 and substituting into (2.5) from (1.5) amd (2.4), we obtain an exact sequence

$$
1 \longrightarrow K^{\times} \longrightarrow W_{K / F} \longrightarrow G_{K / F} \longrightarrow 1 .
$$


Such an exact sequence yields by standard cohomology of groups a member of $H^{2}\left(G_{K / F}, K^{\times}\right)$. Tracking down the isomorphisms that led to (2.6) allows one to identify this cohomology element.

Theorem 2.6. The cohomology class of the exact sequence (2.6) in the group $H^{2}\left(G_{K / F}, K^{\times}\right)$is exactly the canonical generator $u_{K / F}$.

REFERENCE. [Ta3, pp. 4-5].

Theorem 2.6 allows a fairly explicit understanding of $W_{K / F}$ for nonarchimedean local fields.

For the archimedean local fields $\mathbb{R}$ and $\mathbb{C}$, we turn Theorem 2.6 around and use it as a definition of the Weil group. In the case of $\mathbb{R}, H^{2}\left(G_{\mathbb{C} / \mathbb{R}}, \mathbb{C}^{\times}\right)$is cyclic of order 2 , and $W_{\mathbb{C} / \mathbb{R}}$ is defined correspondingly to be a group that fits into a nonsplit exact sequence

$$
1 \longrightarrow \mathbb{C}^{\times} \longrightarrow W_{\mathbb{C} / \mathbb{R}} \longrightarrow G_{\mathbb{C} / \mathbb{R}} \longrightarrow 1 .
$$

Specifically we take $W_{\mathbb{C} / \mathbb{R}}=\mathbb{C}^{\times} \cup j \mathbb{C}^{\times}$, where $j$ acts on $\mathbb{C}^{\times}$by complex conjugation and where $j^{2}=-1 \in \mathbb{C}^{\times}$. In view of (1.7), we make the definition

$$
W_{\mathbb{R}}=W_{\mathbb{C} / \mathbb{R}}=\mathbb{C}^{\times} \cup j \mathbb{C}^{\times} .
$$

Similarly we are led to define $W_{\mathbb{C}}=W_{\mathbb{C} / \mathbb{C}}=\mathbb{C}^{\times}$. With these definitions we readily check that Theorem 2.1 and Corollary 2.5 remain valid for $\mathbb{R}$ and $\mathbb{C}$.

\section{Adeles and Ideles}

Adeles occur in the study of "global fields," which are of two kinds. The global fields of characteristic 0 are the number fields, the finite extensions of $\mathbb{Q}$. The global fields of characteristic nonzero are the finite extensions of the formal rational functions over a finite field. We shall limit our discussion to number fields. General references for adeles are [Cas], [La], and [We4].

The idea with adeles is to study number-theoretic questions about a number field by first studying congruences. For example, to study the factorization of a monic polynomial with integer coefficients, we first study the factorization modulo each prime. In addition, we consider any limitations imposed by treating the polynomial as having real coefficients. Thus, in the case of $\mathbb{Q}$, we use a structure that incorporates congruences modulo each prime (as well as powers of the prime), together with information about $\mathbb{R}$. The ring of adeles $\mathbb{A}_{\mathbb{Q}}$ is the structure in question. We defer to $\S 5$ the way it carries information about factorization of polynomials.

In the case of $\mathbb{Q}$, let $P=\{\infty\} \cup\{$ primes $\}$. For $v \in P$, the field $\mathbb{Q}_{v}$ is to be the field of $p$-adic numbers if $v$ is a prime $p$, and it is to be $\mathbb{R}$ if $v=\infty$. Let $S \subset P$ be a finite set containing $\infty$, and define

$$
\mathbb{A}_{\mathbb{Q}}(S)=\left(\prod_{v \in S} \mathbb{Q}_{v}\right) \times\left(\prod_{v \notin S} \mathbb{Z}_{v}\right) .
$$

With the product topology, $\mathbb{A}_{\mathbb{Q}}(S)$ is a locally compact commutative topological ring. If $S_{1} \subset S_{2}$, then $\mathbb{A}_{\mathbb{Q}}\left(S_{1}\right) \subset \mathbb{A}_{\mathbb{Q}}\left(S_{2}\right)$. The directed system of inclusions allows us to define $\mathbb{A}_{\mathbb{Q}}$ as the direct limit

$$
\mathbb{A}_{\mathbb{Q}}=\lim _{\vec{S}} \mathbb{A}_{\mathbb{Q}}(S)=\bigcup_{S} \mathbb{A}_{\mathbb{Q}}(S) .
$$


The direct-limit topology makes each $\mathbb{A}_{\mathbb{Q}}(S)$ be open in $\mathbb{A}_{\mathbb{Q}}$, the relative topology being the locally compact topology above. Then $\mathbb{A}_{\mathbb{Q}}$ is a locally compact commutative topological ring known as the adeles of $\mathbb{Q}$.

Elements of $\mathbb{A}_{\mathbb{Q}}$ may be regarded as tuples

$$
x=\left(x_{\infty}, x_{2}, x_{3}, x_{5}, \ldots, x_{v}, \ldots\right)=\prod_{v} x_{v}
$$

with almost all (i.e., all but finitely many) $x_{v}$ having $\left|x_{v}\right|_{v} \leq 1$. (Here $|\cdot|_{\infty}$ denotes the module for the local field $\mathbb{R}$, which is just the usual absolute value.) Often one writes simply $x=\left(x_{v}\right)$.

The adeles are the result of a construction called restricted direct product. Suppose that $I$ is a nonempty index set, that $X_{i}$ is a locally compact Hausdorff space for each $i \in I$, and that a compact open subset $K_{i}$ of $X_{i}$ is specified for all $i$ outside a finite subset $S_{\infty}$ of $I$. If $S$ is any finite subset of $I$ containing $S_{\infty}$, we can define

$$
A(S)=\left(\prod_{i \in S} X_{i}\right) \times\left(\prod_{i \notin S} K_{i}\right),
$$

and $A(S)$ will be locally compact Hausdorff. The direct limit $A$ of the $A(S)$ 's as $S$ increases is called the restricted direct product of the $X_{i}$ relative to the $K_{i}$. The space $A$ is locally compact Hausdorff, and each $A(S)$ is open in it. An element of $\prod_{i \in I} x_{i}$ of the Cartesian product $\prod_{i \in I} X_{i}$ is in $A$ if and only if $x_{i}$ is in $K_{i}$ for almost all $i$.

In practice, $X_{i}$ is usually a locally compact group and $K_{i}$ is a compact open subgroup. Then $A$ is a locally compact group. In the case of $\mathbb{A}_{\mathbb{Q}}$, the finite set $S_{\infty}$ is $\{\infty\}$, each $X_{i}$ is a locally compact ring (namely $\mathbb{Q}_{p}$ or $\mathbb{R}$ ), and $K_{i}$ is a compact open subring (namely $\mathbb{Z}_{p}$ ); thus $\mathbb{A}_{\mathbb{Q}}$ is a locally compact ring.

For a general number field $F$ (possibly $\mathbb{Q}$ itself), we construct the ring $\mathbb{A}_{F}$ of adeles of $F$ as follows. A completion of $F$ is a pair $(\lambda, K)$, where $K$ is a local field and $\lambda: F \rightarrow K$ is a field map with dense image. Two completions $(\lambda, K)$ and $\left(\lambda^{\prime}, K^{\prime}\right)$ are equivalent is there is a topological isomorphism $\rho: K \rightarrow K^{\prime}$ such that $\rho \circ \lambda=\lambda^{\prime}$. A place is an equivalence class of completions. Places are typically denoted $v$, and a representative of the corresponding local field is denoted $F_{v}$. When $F=\mathbb{Q}$, the only places are those coming from embedding $\mathbb{Q}$ in $\mathbb{R}$ and in each $\mathbb{Q}_{p}$ for $p$ prime.

An isomorphism that exhibits two completions as corresponding to the same place preserves the module. Consequently restriction of the module to $F$ gives a well defined function $|\cdot|_{v}$ on $F$.

Suppose that $F^{\prime} / F$ is an extension of number fields, and let $w$ be a place of $F^{\prime}$. Regard $w$ as a field map $w: F^{\prime} \rightarrow F_{w}^{\prime}$. It is not hard to see that the closure $\overline{w(F)}$ of $w(F)$ is a local field, that $F_{w}^{\prime}$ is a finite algebraic extension of $\overline{w(F)}$, and that the restriction of $w$ to $F$ determines a place $v$ of $F$. In this case we say that $v$ is the place of $F$ that lies below $w$, and that $w$ lies above $v$. We write $w \mid v$.

Theorem 3.1. Let $F^{\prime} / F$ be an extension of number fields, and let $v$ be a place of $k$. Then there exists a place of $F^{\prime}$ lying above $v$, and there are only finitely many such places.

REFERENCE. [We4, p. 45]. 
We can apply Theorem 3.1 to construct $\mathbb{A}_{F}$. The $X_{i}$ are the various $F_{v}$. All of these are $p$-adic fields except those with $v$ lying above the place $\infty$ of $\mathbb{Q}$, and Theorem 3.1 says that there are only finitely many such places. We take $S_{\infty}$ to be the set of places lying above $\infty$, and we let the $K_{i}$ 's be the rings of integers $\mathcal{O}_{v}$ in $F_{v}$. Then $\mathbb{A}_{F}$ is the restricted direct product of the $F_{v}$ relative to the $K_{v}$, and it is a locally compact commutative ring.

We can get some insight into the places of a number field $F$ by treating $F$ as an extension of $\mathbb{Q}$ and considering all places lying over a place of $\mathbb{Q}$ (a prime or $\infty$ ). By the theory of semisimple algebras, the algebra $F \otimes \mathbb{Q}_{v}$ over $\mathbb{Q}_{v}$ is a finite direct sum of fields, each of which is a finite extension of $\mathbb{Q}_{v}$. Fix an algebraic closure $\overline{\mathbb{Q}}_{v}$, and consider the set of field maps $\operatorname{Hom}_{\mathbb{Q}}\left(F, \overline{\mathbb{Q}}_{v}\right)$. The group $D_{v}=\operatorname{Gal}\left(\overline{\mathbb{Q}}_{v} / \mathbb{Q}_{v}\right)$ acts on this set of maps by acting on the values of each map. Let $P_{v}(F)=D_{v} \backslash \operatorname{Hom}_{\mathbb{Q}}\left(F, \overline{\mathbb{Q}}_{v}\right)$. Then

$$
F \otimes_{\mathbb{Q}} \mathbb{Q}_{v} \cong \bigoplus_{w \in P_{v}(F)} F_{w}
$$

with the right side involving each place lying over $v$ just once.

Let us consider this decomposition for the infinite places, those lying above $\infty$ of $\mathbb{Q}$. We shall write $F_{\infty}$ for the algebra $F \otimes_{\mathbb{Q}} \mathbb{R}$. This $\mathbb{R}$ algebra is of the form $\mathbb{R}^{r_{1}} \times \mathbb{C}^{r_{2}}$ for some integers $r_{1} \geq 0$ and $r_{2} \geq 0$ satisfying $r_{1}+2 r_{2}=[F: \mathbb{Q}]$. Since $D_{\infty}=\operatorname{Gal}(\mathbb{C} / \mathbb{R})$ consists of 1 and complex conjugation, $P_{\infty}(F)$ consists of the set $\operatorname{Hom}_{\mathbb{Q}}(F, \mathbb{C})$ of embeddings of $F$ into $\mathbb{C}$, with two embeddings identified when they are complex conjugates of one another. Thus $r_{1}$ is the number of embeddings into $\mathbb{R}$, and $r_{2}$ is the number of complex-conjugate pairs of nonreal embeddings of $F$ into $\mathbb{C}$.

Next let us consider the finite places. Recall that the ring of integers $\mathcal{O}$ in $F$ consists of all elements satisfying a monic polynomial equation with $\mathbb{Z}$ coefficients. The places lying over the primes of $\mathbb{Q}$ are related to the nontrivial prime ideals of $\mathcal{O}$. Let $v$ be a place of $F$ lying above a prime $p$ for $\mathbb{Q}$. It is easy to see that the mapping $F \rightarrow F_{v}$ carries $\mathcal{O}$ into the ring of integers $\mathcal{O}_{v}$ of $F_{v}$. Let $\mathfrak{m}_{v}$ be the maximal ideal of $\mathcal{O}_{v}$, and let $P_{v}$ be the inverse image in $\mathcal{O}$ of $\mathfrak{m}_{v}$. Then $P_{v}$ is a prime ideal of $\mathcal{O}$. That is, every finite place of $F$ leads to a prime ideal of $\mathcal{O}$.

This fact admits a converse. Before stating the converse, we recall that the nonzero ideals of $\mathcal{O}$ admit unique factorizations as products of prime ideals, the exponents of the prime ideals being integers $\geq 0$. The notion of ideal can be extended to fractional ideal; a fractional ideal is just a set of the form $n^{-1} I$ for an ideal $I$ and some nonzero $n \in \mathbb{Z}$. It is not hard to see that the nonzero fractional ideals form a group. Consequently the nonzero fractional ideals admit unique factorizations as products of prime ideals, the exponents of the prime ideals being integers that are not necessarily $\geq 0$.

Theorem 3.2. Let $P$ be a nontrivial prime ideal of $\mathcal{O}$, and let $q=|\mathcal{O} / P|$. For each $x \in F^{\times}$, let $v(x)$ be the power of $P$ that appears in the factorization of the principal fractional ideal $(x)=x \mathcal{O}$, and define $|x|_{P}=q^{-v(x)}$. Then $|\cdot|_{P}$ defines a metric on $F$, and the completion of $F$ in this metric is a local field whose module is the continuous extension of $|\cdot|_{P}$. The result is a place of $F$, and the prime ideal of $\mathcal{O}$ associated to this place is just $P$.

Since the factorization of $(x)$ in the theorem is finite, it follows that $|x|_{v}$ is different from 1 for only finitely many places $v$. One consequence of this fact is 
that any $x \in F$ embeds diagonally as an element of $\mathbb{A}_{F}$. We shall make constant use of this embedding. It is tempting to write $\operatorname{diag} F$ for the image, but this notation soon becomes unwieldy and it is customary to denote the image simply by $F$. Briefly the subset $F$ of $\mathbb{A}_{F}$ always means the diagonally embedded version of $F$ unless the contrary is stated.

A second consequence of the fact that $|x|_{v} \neq 1$ for only finitely many $v$ is that $\prod_{v}|x|_{v}$ is well defined for each $x \in F^{\times}$. The next theorem tells the value of this product.

Theorem 3.3 (Artin product formula). $\prod_{v}|x|_{v}=1$ for all $x \in F^{\times}$.

REFERENCE. [La, p. 99].

Theorem 3.4. The image of $F$ in $\mathbb{A}_{F}$ is discrete, and the quotient group $\mathbb{A}_{F} / F$ is compact.

REFERENCE. [La, p. 139].

The construction of the ideles of the number field $F$ is a second use of the notion of a restricted direct product. The index set $I$ is the set $\{v\}$ of places, the factors $X_{i}$ are the multiplicative groups $F_{v}^{\times}$, the subset $S_{\infty}$ is the set of infinite places, and the $K_{i}$ are the groups of units $\mathcal{O}_{v}^{\times}$. The restricted direct product is a locally compact abelian group denoted $\mathbb{A}_{F}^{\times}$and called the ideles of $F$.

If $x$ is in $F^{\times}$, then the tuple consisting of $x$ in every place is an idele as a consequence of the discussion before Theorem 3.3. Hence $F^{\times}$embeds diagonally in $\mathbb{A}_{F}^{\times}$. We denote the image simply by $F^{\times}$, understanding that $F^{\times}$is diagonally embedded unless the contrary is stated.

If one tracks down the definitions, the topology on $\mathbb{A}_{F}^{\times}$is not the relative topology from $\mathbb{A}_{F}$ but is finer. Actually $\mathbb{A}_{F}^{\times}$gets the relative topology from

$$
\left\{(x, y) \in \mathbb{A}_{F} \times \mathbb{A}_{F} \mid x y=1\right\} .
$$

Since $F^{\times}$is discrete in $\mathbb{A}_{F}$ (Theorem 3.4) and since the topology on $\mathbb{A}_{F}^{\times}$is finer than the relative topology on $\mathbb{A}_{F}, F^{\times}$is discrete in $\mathbb{A}_{F}^{\times}$.

Being a locally compact abelian group, $\mathbb{A}_{F}$ has a Haar measure $\mu_{\mathbb{A}_{F}}$, and this can evidently be taken to be the product of the Haar measure on each $F_{v}$ if the latter are normalized at almost every place to assign mass one to $\mathcal{O}_{v}$. Multiplication by any $r \in \mathbb{A}_{F}^{\times}$is an automorphism of $\mathbb{A}_{F}$ and hence carries $\mu_{\mathbb{A}_{F}}$ to a multiple of itself. We write

$$
d \mu_{\mathbb{A}_{F}}(r x)=|r|_{\mathbb{A}_{F}} d \mu_{\mathbb{A}_{F}}(x)
$$

and call $|r|_{\mathbb{A}_{F}}$ the module of $r$. Arguing first with $r$ equal to 1 in all but one place and then passing to general $r$, we see that $|r|_{\mathbb{A}_{F}}=\prod_{v}\left|r_{v}\right|_{v}$. Theorem 3.3 therefore implies that any element $x$ of $F^{\times}$has $|x|_{\mathbb{A}_{F}}=1$.

Let $\left(\mathbb{A}_{F}^{\times}\right)^{1}$ be the subgroup of elements of $\mathbb{A}_{F}^{\times}$of module 1 , i.e., the kernel of the homomorphism $|\cdot|_{\mathbb{A}_{F}}: \mathbb{A}_{F}^{\times} \rightarrow \mathbb{R}_{+}^{\times}$. We have just seen that $F^{\times}$lies in this subgroup, and we saw above that $F^{\times}$is discrete. This proves the easy half of the following theorem.

Theorem 3.5. The diagonal embedding $F^{\times} \rightarrow \mathbb{A}_{F}^{\times}$carries $F^{\times}$to a discrete subgroup of $\left(\mathbb{A}_{F}^{\times}\right)^{1}$, and the quotient $\left(\mathbb{A}_{F}^{\times}\right)^{1} / F^{\times}$is compact. 
Remarks. The compactness of the quotient is closely related to the Dirichlet Unit Theorem. For a proof of the result and a discussion of the connection, see [Cas, pp. 70-73] and [La, pp. 142-146]. For a direct argument in a more general context, see [We4, p. 76].

The relationship between $\mathbb{A}_{F}^{\times}$and $\left(\mathbb{A}_{F}^{\times}\right)^{1}$ is that

$$
\mathbb{A}_{F}^{\times}=\left(\mathbb{A}_{F}^{\times}\right)^{1} \times R
$$

for a noncanonical subgroup $R$ isomorphic to $\mathbb{R}_{+}^{\times}$. In fact, fix an infinite place $v$. Then $F_{v}^{\times}$contains a subgroup $\mathbb{R}_{+}^{\times}$, and we let $R$ be the image of this subgroup in $\mathbb{A}_{F}^{\times}$under inclusion into the $v^{\text {th }}$ place. It is clear that the module map $|\cdot| \mathbb{A}_{F}$ carries $R$ one-one onto $\mathbb{R}_{+}^{\times}$. Thus (3.2) follows.

From (3.2) and Theorem 3.5, it follows that

$$
\mathbb{A}_{F}^{\times} / H \text { is compact }
$$

for any subgroup $H$ of $\mathbb{A}_{F}^{\times}$containing $R F^{\times}$. Recall that $F_{\infty}=F \otimes_{\mathbb{Q}} \mathbb{R}$ gives the archimedean component of $\mathbb{A}_{F}$ (with 1 in all finite places). Then $F_{\infty}^{\times}$gives the archimedean component of $\mathbb{A}_{F}^{\times}$. Since $F_{\infty}^{\times}$contains $R$, (3.3) implies that $\mathbb{A}_{F}^{\times} /\left(F_{\infty}^{\times} F^{\times}\right)$is compact. Let $U_{1}$ be the ring $\prod_{v \text { finite }} \mathcal{O}_{v}$, so that $K_{1}=U_{1}^{\times}$is just $\prod_{v \text { finite }} \mathcal{O}_{v}^{\times}$.

Corollary 3.6. Suppose that $K$ is any open compact subgroup of $K_{1}$. Then the set $X_{K}=F^{\times} \backslash \mathbb{A}_{F}^{\times} /\left(F_{\infty}^{\times} K\right)$ is finite.

REMARK. When $K=K_{1}$, use of the relationship discussed below between ideles and fractional ideals allows one to identify $X_{K_{1}}$ with the ideal class group of $F$. Thus the corollary gives an adelic proof of the finiteness of the class number.

Proof. The above considerations show that $\mathbb{A}_{F}^{\times} /\left(F_{\infty}^{\times} F^{\times}\right)$is compact, and hence $X_{K}$ is compact. On the other hand, $F_{\infty}^{\times} K$ is open in $\mathbb{A}_{F}^{\times}$. Thus $\mathbb{A}_{F}^{\times} /\left(F_{\infty}^{\times} K\right)$ is discrete, and consequently $X_{K}$ is discrete.

Historically, ideles were introduced before adeles. Chevalley's purpose in introducing ideles was to extend class field theory to infinite abelian extensions. But, as is indicated in the introduction of [Ch], the theory of ideles served also the purpose of reinterpreting results about fractional ideals and related notions. We give some details about this point in order to prepare for Artin reciprocity, which will be discussed in the next section. For each $x=\left(x_{v}\right) \in \mathbb{A}_{F}^{\times}$, we create a fractional ideal as follows. Each finite place $v$ corresponds to some prime ideal $\wp$ in $\mathcal{O}$. With $q_{v}=\left|\mathcal{O}_{v} / \mathfrak{m}_{v}\right|$ and $\left|x_{v}\right|_{v}=q_{v}^{-v\left(x_{v}\right)}$, associate to $x$ the fractional ideal $\prod_{\wp} \wp^{v\left(x_{v}\right)}$. If $x=\left(x_{0}, x_{0}, \ldots\right)$ is in $F^{\times}$, then this definition reproduces the fractional ideal $\left(x_{0}\right)$ in its factored form; hence there is no ambiguity if we refer to this fractional ideal as $(x)$ in every case. If $S$ is a finite set of places containing $S_{\infty}$ and if $x$ is an idele, we define $(x)^{S}=\prod_{\wp \notin S} \wp^{v\left(x_{v}\right)}$.

Artin reciprocity initially involves a homomorphism of fractional ideals into an abelian Galois group, and we shall want to lift this homomorphism to a homomorphism of the group of ideles. We shall impose a continuity condition. Let $S$ be a finite set of places containing $S_{\infty}$, and let $I^{S}$ be the subgroup of fractional ideals whose $\wp$ factor is (1) for every finite $\wp \in S$. If $G$ is an abelian topological group, we 
say that a homomorphism $\varphi: I^{S} \rightarrow G$ is admissible if, for each neighborhood $N$ of 1 in $G$, there exists $\delta>0$ such that $\varphi\left((a)^{S}\right)$ is in $N$ whenever $a$ is a member of $F^{\times}$such that $|a-1|_{v}<\delta$ for all $v \in S$ (including all infinite places). To reinterpret admissible $\varphi$ 's in terms of ideles, we use the following Weak Approximation Theorem.

Theorem 3.7. Let $|\cdot|_{n}, 1 \leq n \leq N$ be distinct places of $F$, finite or infinite. If $x_{n}, 1 \leq n \leq N$, are members of $F$ and if $\epsilon>0$ is given, then there exists $\xi \in F^{\times}$ with $\left|\xi-x_{n}\right|_{n}<\epsilon$ for $1 \leq n \leq N$.

Reference. [Cas, p. 48].

Theorem 3.8. Let $S$ be a finite set of places containing $S_{\infty}$, and let $G$ be a compact abelian group (usually finite abelian or $S^{1}$ ). If $\varphi: I^{S} \rightarrow G$ is an admissible homomorphism, then there exists a unique homomorphism $\tilde{\varphi}: \mathbb{A}_{F}^{\times} \rightarrow G$ such that

(a) $\tilde{\varphi}$ is continuous

(b) $\tilde{\varphi}$ is 1 on $F^{\times}$

(c) $\tilde{\varphi}(x)=\varphi\left((x)^{S}\right)$ for all $x=\left(x_{v}\right) \in \mathbb{A}_{F}^{\times}$such that $x_{v}=1$ for all $v \in S$ (including all infinite places).

REFERENCE. [Ta2, pp. 169-170].

Proof of Uniqueness. Given $x \in \mathbb{A}_{F}^{\times}$, choose, by Theorem 3.7, a sequence of elements $a_{n}$ of $F^{\times}$such that $a_{n} \rightarrow x^{-1}$ at the places of $S$. For $y \in \mathbb{A}_{F}^{\times}$, let $y^{S}$ be the idele with all entries in places of $S$ set equal to 1 , and let $y_{S}$ be the idele with all entries in places outside $S$ set equal to 1 . For every $n$, we have

$$
\begin{aligned}
\tilde{\varphi}(x) & =\tilde{\varphi}\left(a_{n} x\right) & & \text { by (b) } \\
& =\tilde{\varphi}\left(\left(a_{n} x\right)_{S}\right) \tilde{\varphi}\left(\left(a_{n} x\right)^{S}\right) & & \text { since } \tilde{\varphi} \text { is a homomorphism } \\
& =\tilde{\varphi}\left(\left(a_{n} x\right)_{S}\right) \varphi\left(\left(a_{n} x\right)^{S}\right) & & \text { by (c). }
\end{aligned}
$$

The first factor on the right side tends to 1 by (a). Thus

$$
\tilde{\varphi}(x)=\lim _{n \rightarrow \infty} \varphi\left(\left(a_{n} x\right)^{S}\right) .
$$

\section{Artin Reciprocity}

A general reference for Artin reciprocity is [Ta2]. Let $K / F$ be a Galois extension of number fields, and let $G=\operatorname{Gal}(K / F)$ be the Galois group.

Let $v$ be a finite place of $F$, and let $\wp$ be the corresponding prime ideal of $\mathcal{O}_{F}$. The ideal $\wp \mathcal{O}_{K}$ of $\mathcal{O}_{K}$ has a factorization into prime ideals of $\mathcal{O}_{K}$, say

$$
\wp \mathcal{O}_{K}=P_{1}^{e_{1}} \cdots P_{g}^{e_{g}}
$$

with the $P_{j}$ distinct and with all $e_{j}>0$. Here the $P_{j}$ 's are exactly the prime ideals of $\mathcal{O}_{K}$ that contain $\wp$, and each $P_{j}$ has $P_{j} \cap \mathcal{O}_{F}=\wp$. We say that the $P_{j}$ lie above $\wp$. From the correspondence of finite places to prime ideals, it is easy to see that the places $w_{j}$ corresponding to the $P_{j}$ 's are exactly the places that lie above $v$. 
Theorem 4.1. In the factorization $\wp \mathcal{O}_{K}=P_{1}^{e_{1}} \cdots P_{g}^{e_{g}}$ into prime ideals in $\mathcal{O}_{K}$, the Galois group $G$ permutes $P_{1}, \ldots, P_{g}$, and the action of $G$ on the g-element set is transitive. Consequently $e_{1}=\cdots=e_{g}$. Moreover, if $\left|\mathcal{O}_{F} / \wp\right|=q$ and $\left|\mathcal{O}_{K} / P_{j}\right|=q^{f_{j}}$, then $f_{1}=\cdots=f_{g}$. If e denotes the common value of the $e_{j}$ and if $f$ denotes the common value of the $f_{j}$, then

$$
\text { efg }=[K: F] \text {. }
$$

The prime ideal $\wp$ of $\mathcal{O}_{F}$ is said to ramify in $K$ if $e>1$. Ramification is an exceptional occurrence: If $v$ lies above the place $p$ of $\mathbb{Q}$, then ramification of $\wp$ in $K$ implies that $p$ divides the absolute discriminant of $K$. In particular, only finitely many prime ideals of $\mathcal{O}_{F}$ ramify in $K$.

In the situation of the theorem, let $P$ be one of $P_{1}, \ldots, P_{g}$. Let $w$ and $v$ be the places of $K$ and $F$ corresponding to $P$ and $\wp$, so that $w$ lies above $v$. Write $K_{P}$ and $F_{\wp}$ for the completions. Define

$$
G_{P}=\{\sigma \in G \mid \sigma(P)=P\} .
$$

This group is called the decomposition group relative to $P$. The members of $G_{P}$ acts as isometries of $K$ in the norm $|\cdot|_{P}$. Consequently $\sigma$ extends to an automorphism of $K_{P}$, and we see that we can think in terms of an inclusion

$$
G_{P} \hookrightarrow \operatorname{Gal}\left(K_{P} / F_{\wp}\right) .
$$

Theorem 4.2. The embedding of $G_{P}$ in (4.1) is onto $\operatorname{Gal}\left(K_{P} / F_{\wp}\right)$, and $K_{P} / F_{\wp}$ is a Galois extension of local fields.

We observed before Theorem 1.1 that each member of $\operatorname{Gal}\left(K_{P} / F_{\wp}\right)$ acts by an isometry and consequently induces an automorphism of $\operatorname{Gal}\left(K_{P} / F_{\wp}\right)$ into $\operatorname{Gal}\left(k_{P}^{\prime} / k_{\wp}\right)$, where $k_{P}^{\prime}$ and $k_{\wp}$ are the respective residue fields. Theorem 1.1 says that the resulting homomorphism is onto. Thus we can rewrite the exact sequence (1.1) in this context as

$$
1 \longrightarrow I_{P} \longrightarrow G_{P} \longrightarrow \operatorname{Gal}\left(k_{P}^{\prime} / k_{\wp}\right) \longrightarrow 0,
$$

where the inertia group $I_{P}$ is defined to be the kernel. With $e, f$, and $g$ as in Theorem 4.1, we know that efg $=[K: F]$. Since $G$ acts transitively on $P_{1}, \ldots, P_{g}$ by Theorem 4.1, the isotropy subgroup $G_{P}$ at $P$ has $e f g / g=e f$ elements. By Theorem 4.2, $\left|\operatorname{Gal}\left(K_{P} / F_{\wp}\right)\right|=e f$. Our definitions make $\left|\operatorname{Gal}\left(k_{P}^{\prime} / k_{\wp}\right)\right|=f$, and therefore $\left|I_{P}\right|=e$.

In other words, if $\wp$ is unramified in $K$ (as is the case for almost all prime ideals of $F)$, the extension $K_{P} / F_{\wp}$ is unramified in the sense of $\S 1$. In this case, $\operatorname{Gal}\left(K_{P} / F_{\wp}\right)$ contains a well defined Frobenius element, as in the definition following Theorem 1.2. If $P$ is replaced by another prime ideal $P^{\prime}$ lying over $\wp$, then $G_{P}$ is conjugate to $G_{P^{\prime}}$ by an element of $G$ carrying $P$ to $P^{\prime}$, and this conjugacy carries the Frobenius element to the Frobenius element. In terms of $\wp$ as a given piece of data, the Frobenius element is then any element of a certain conjugacy class of $G$.

Artin reciprocity deals with the situation that $G=\operatorname{Gal}(K / F)$ is abelian. In this case when $\wp$ is a prime ideal of $\mathcal{O}_{F}$ that is unramified in $K$, the conjugacy class of Frobenius elements reduces to a single element, and we can unambiguously denote the Frobenius element by the notation

$$
\left[\frac{K / F}{\wp}\right] \in G .
$$


Let $S$ be the finite set of all infinite places of $F$ and all finite places of $F$ that ramify in $K$. If $X=\prod_{j} \wp_{j}^{n_{j}}$ is the factorization of a fractional ideal of $F$ into primes, we recall that $X^{S}=\prod_{j \notin S} \wp_{j}^{n_{j}}$. Then we can define

$$
\left[\frac{K / F}{X^{S}}\right]=\prod_{j \notin S}\left[\frac{K / F}{\wp_{j}}\right]^{n_{j}} .
$$

The resulting homomorphism of $I^{S}$ into $G$ is called the Artin symbol of $K / F$.

Theorem 4.3 (Artin reciprocity, first form). Let $K / F$ be a finite abelian Galois extension of degree $n$, and let $S$ be the finite set of all infinite places of $F$ and all finite places of $F$ that ramify in $K$. If $a \in F^{\times}$is such that a is in $\left(F_{v}^{\times}\right)^{n}$ for every $v \in S$ and if $(a)$ denotes the fractional ideal $a \mathcal{O}_{F}$, then

$$
\left[\frac{K / F}{(a)^{S}}\right]=1
$$

REFEREnCE. [Ta2, p. 167].

This first form of Artin reciprocity is the weakest of three forms that we shall consider. However, it is already strong enough so that with a little computation it implies quadratic reciprocity [Cas-Fr, pp. 348-350]. It also implies a more general $m^{\text {th }}$ power reciprocity theorem due to Kummer.

We shall now sharpen the statement of Artin reciprocity so as to be able to bring Theorem 3.8 to bear.

Theorem 4.4 (Artin reciprocity, second form). Let $K / F$ be a finite abelian Galois extension, and let $S$ be the finite set of all infinite places of $F$ and all finite places of $F$ that ramify in $K$. There exists $\delta>0$ such that whenever $a \in F^{\times}$has $|a-1|_{v}<\delta$ for all $v \in S$, then

$$
\left[\frac{K / F}{(a)^{S}}\right]=1
$$

REFERENCE. [Ta2, p. 167].

Theorem 4.4 implies Theorem 4.3 by a simple argument [Ta2, p. 167] using the Weak Approximation Theorem (Theorem 3.7).

In the terminology at the end of $\S 3$, Theorem 4.4 says that the homomorphism $I^{S} \rightarrow G$ defined by the Artin symbol is admissible. By Theorem 3.8, the Artin symbol lifts uniquely to a continuous homomorphism of the idele class group of $F$,

$$
C_{F}=\mathbb{A}_{F}^{\times} / F^{\times}
$$

into $G$. Let us call this homomorphism the Artin map of $K / F$ and denote it by $\theta_{K / F}: C_{F} \rightarrow G$.

Theorem 4.5. Let $K / F$ be a finite abelian Galois extension, let $v$ be a place of $F$, and let $w$ be a place of $K$ lying above $v$. If $i_{v}: F_{v}^{\times} \rightarrow C_{F}$ denotes the composition of inclusion of $F_{v}$ into the $v^{\text {th }}$ place of $\mathbb{A}_{F}^{\times}$followed by the quotient map to $C_{F}$, then the Artin map and the local reciprocity map are related by $\theta_{K / F} \circ i_{v}=\theta_{K_{w} / F_{v}}$ as homomorphisms $F_{v}^{\times} \rightarrow G$. 
REFERENCE. [Ta2, p. 175].

Theorems 4.4 and 4.5 are the main facts about the Artin symbol and Artin map that we need in the next section. For completeness we include a little more information at this time. It is apparent from Theorem 4.5 and the precise statement of local class field theory in Theorem 2.4 that there has to be a sharper statement of Artin reciprocity than in Theorem 4.4. Here is such a result.

Theorem 4.6 (Artin reciprocity, third form). Let $K / F$ be a finite abelian Galois extension, and let $S$ be the finite set of all infinite places of $F$ and all finite places of $F$ that ramify in $K$. If an element $a \in F^{\times}$is a norm from $K_{w}$ for all $w$ lying over places of $S$, then

$$
\left[\frac{K / F}{(a)^{S}}\right]=1
$$

REFERENCE. [Ta2, p. 176].

Now we think of $K$ as varying. Namely we fix an algebraic closure $\bar{F}$ of $F$ and consider finite abelian Galois extensions $K$ of $F$ lying in $\bar{F}$. Let $G_{F}$ be the Galois group of $\bar{F}$ over $F$, and let $G_{F}^{\mathrm{ab}}=G_{F} / G^{c}$ be the Galois group of the maximal abelian extension of $F$. If $K$ is a finite abelian extension of $F$, then the Artin map $\theta_{K / F}$ is a continuous homomorphism of $C_{F}$ into $G_{K / F}=\operatorname{Gal}(K / F)$. These homomorphisms have a compatibility property that allows us to lift them to a single continuous homomorphism with values in the inverse limit, namely $\theta_{F}: C_{F} \rightarrow G_{F}^{\mathrm{ab}}$. We call $\theta_{F}$ the Artin map of $F$. It follows from the various compatibility properties that Theorem 4.5 can be restated in this notation as

$$
\theta_{F} \circ i_{v}=\theta_{F_{v}}
$$

as homomorphisms $F_{v}^{\times} \rightarrow G_{F}^{\text {ab }}$.

Lemma. The Artin map $\theta_{F}$ carries $C_{F}$ onto $G_{F}^{\mathrm{ab}}$.

Sketch of proof. By $(3.2), \mathbb{A}_{F}^{\times}=R \times\left(\mathbb{A}_{F}^{\times}\right)^{1}$. Since $F^{\times}$lies in $\left(\mathbb{A}_{F}^{\times}\right)^{1}$, we obtain $C_{F} \cong R \times C_{F}^{1}$, where $C_{F}^{1}=\left(\mathbb{A}_{F}^{\times}\right)^{1} / F^{\times}$. Since $R$ is connected and $G_{F}^{\text {ab }}$ is totally disconnected, $\theta_{F}(R)=1$. Thus $\theta_{F}\left(C_{F}\right)=\theta_{F}\left(C_{F}^{1}\right)$, and this is compact by Theorem 3.5. On the other hand, one shows that the composition of $\theta_{F}$ and passage to any finite quotient of $G_{F}^{\mathrm{ab}}$ carries $C_{F}$ onto the finite quotient. Hence $\theta_{F}\left(C_{F}\right)$ is dense in $G_{F}^{\mathrm{ab}}$.

In practice, Artin reciprocity is proved at the same time as the Existence Theorem of global class field theory, whose statement is given in Theorem 4.7 below. If $v$ is a place of $F$ and $w$ is a place of $K$ lying above $v$, then the norm map $N_{K_{w} / F_{v}}: K_{w}^{\times} \rightarrow F_{v}^{\times}$is well defined. We set $N_{K / F}: \mathbb{A}_{K}^{\times} \rightarrow \mathbb{A}_{F}^{\times}$equal to the coordinate-by-coordinate product $N_{K / F}=\prod_{w} N_{K_{w} / F_{v}}^{\times}$. It is clear that $N_{K / F}$ carries diagonally embedded $K^{\times}$to diagonally embedded $F^{\times}$and therefore descends to a homomorphism $N_{K / F}: C_{K} \rightarrow C_{F}$.

Theorem 4.7. The map $K \mapsto C_{F} / N_{K / F}\left(C_{K}\right)$ is a bijection of the set of finite abelian Galois extensions $K$ of $F$ onto the set of open subgroups of $C_{F}$ of finite index. The field corresponding to a subgroup $B$ is the fixed field of the subgroup $\theta_{F}(B)$ of $G_{F}^{\mathrm{ab}}$. 
REFERENCES. [Ta2, p. 172] and [Ar-Ta, p. 70].

Corollary 4.8. The kernel of the Artin map $\theta_{F}$ of $C_{F}$ onto $G_{F}^{\mathrm{ab}}$ is the identity component $\left(C_{F}\right)_{0}$ of $C_{F}$.

RefERENCE. [Ta2, p. 173].

We can summarize Corollary 4.8 as saying that the sequence

$$
1 \longrightarrow\left(C_{F}\right)_{0} \longrightarrow C_{F} \stackrel{\theta_{F}}{\longrightarrow} G_{F}^{\mathrm{ab}} \longrightarrow 0
$$

is exact.

\section{Artin $L$ Functions}

At the beginning of $\S 3$, we mentioned that the ring of adeles carries information about the factorization of polynomials, and we shall elaborate on this assertion now. The Artin $L$ functions to be introduced in this section encode this information as explicit functions of a complex variable given by product formulas. Artin reciprocity enables one to recognize certain Artin $L$ functions as arising in another way that shows that they have nice analytic properties.

Example 1. For the polynomial $R(X)=X^{2}+1$, we ask how $R(X)$ reduces modulo $p$ for primes $p \neq 2$. Before giving the well known answer, let us encode the problem in a generating function. Put

$$
\left(\frac{-1}{p}\right)= \begin{cases}+1 & \text { if } X^{2}+1 \text { factors completely modulo } p \\ -1 & \text { if } X^{2}+1 \text { is irreducible modulo } p\end{cases}
$$

and define

$$
L(s)=\prod_{p \neq 2} \frac{1}{1-\left(\frac{-1}{p}\right) p^{-s}} .
$$

This certainly converges for $\operatorname{Re} s>1$. The well known answer to our question amounts to giving the pattern for $\left(\frac{-1}{p}\right)$, which is

$$
\left(\frac{-1}{p}\right)= \begin{cases}+1 & \text { if } p=4 k+1 \\ -1 & \text { if } p=4 k-1\end{cases}
$$

This is the simplest case of quadratic reciprocity. The point to observe is that the pattern is described by finitely many linear congruences. If we define

$$
\chi(n)= \begin{cases}+1 & \text { if } n \equiv 1 \bmod 4 \\ -1 & \text { if } n \equiv 3 \bmod 4 \\ 0 & \text { if } n \text { even }\end{cases}
$$

then

$$
L(s)=\prod_{p} \frac{1}{1-\chi(p) p^{-s}} .
$$

From the property $\chi\left(m m^{\prime}\right)=\chi(m) \chi\left(m^{\prime}\right)$, we obtain

$$
L(s)=\sum_{n=1}^{\infty} \frac{\chi(n)}{n^{s}} .
$$


In this form, $L(s)$ becomes more manageable. This series is absolutely convergent for $\operatorname{Re} s>1$. Use of summation by parts shows that $L(s)$ converges for $\operatorname{Re} s>0$, and an elementary argument shows that $L(s)$ continues to an entire function. It is not hard to see that $L(1) \neq 0$, and from this fact it follows that there are infinitely many primes $p=4 k+1$ and infinitely many primes $p=4 k-1$. In other words, interesting information about primes has been encoded in $L(s)$ at a spot on the boundary of the region where $L(s)$ converges absolutely. Finding the pattern for $\frac{1}{1-\left(\frac{-1}{p}\right)}$ enables us to extract this information.

Example 2 ([Buhl] and $[\operatorname{Lgl} 6])$. Let us consider the polynomial

$$
R(X)=X^{5}+10 X^{3}-10 X^{2}+35 X-18 .
$$

This has discriminant $2^{6} 5^{8} 11^{2}$, and the question is to find the pattern of how $R(X)$ reduces modulo $p$ for $p \neq 2,5,11$. For example, we can readily find by computer that $R(X)$ is irreducible modulo $p$ for $p=7,13,19,29,43,47,59, \ldots$ Similarly we find that $R(X)$ splits completely for $p=2063,2213,2953,3631, \ldots$. What is the pattern? These sequences of primes are not related to linear congruences, and the Langlands theory gives conjectures that describe the pattern. Let $F$ be the splitting field of $R(X)$ over $\mathbb{Q}$, and let $G$ be the Galois group. Since the discriminant is a square, $G \subset A_{5}$. The group $G$ contains a Frobenius element $\operatorname{Fr}_{p}$ for each $p \neq 2,5,11$, and this element is the lift to $G_{p}$ of a generator of the Galois group of $R(X) \bmod p$. Modulo $p=7, R(X)$ is irreducible; so $G$ has an element of order 5 . Modulo $p=3$, $R(X)$ is the product of two linear factors and an irreducible cubic; so $G$ has an element of order 3 . Since $A_{5}$ is generated by any two elements of respective orders 5 and 3, we conclude that $G=A_{5}$.

For any $p \neq 2,5,11$, the Galois group of $R(X) \bmod p$ tells us a great deal about the factorization of $R(X) \bmod p$. The generator of this group is a Frobenius element, which can be any element in a particular conjugacy class of $G$. In the case of $A_{5}$, the order of an element determines the conjugacy class of the element in $A_{5}$ unless the order is 5 , for which there are two conjugacy classes. The order of the Frobenius element is $f$. Order 4 does not occur in $A_{5}$, and thus $f=1,2$, 3 , or 5 . If $f=5, R(X) \bmod p$ is irreducible. If $f=3, R(X) \bmod p$ is the product of two distinct linear factors and an irreducible cubic. If $f=2$, the element of $G$ has to be the product of two 2-cycles; thus $R(X) \bmod p$ has to be the product of a linear factor and two distinct irreducible quadratic factors. Finally if $f=1$, then $R(X) \bmod p$ splits into five distinct linear factors. The value of $f$ determines the conjugacy class of Frobenius elements in $A_{5}$ completely unless $f=5$. For $f=5$, there are two conjugacy classes; see [Buhl, p. 53] for how to distinguish them.

In order to encode the full information about the conjugacy classes of the Frobenius elements in one or more generating functions, we can proceed as follows. Let $\sigma$ be a finite-dimensional representation of $G$ over $\mathbb{C}$. Then the generating function is

$$
L(s, \sigma)=\prod_{p \neq 2,5,11} \frac{1}{\operatorname{det}\left(1-p^{-s} \sigma\left(\operatorname{Fr}_{p}\right)\right)}, .
$$

except that suitable factors for $p=2,5,11$ need to be included. The goal is to recognize this function in another form and thereby to find the pattern of the coefficients. This is carried out in [Buhl]. 
Let $K / F$ be a Galois extension of number fields, with Galois group $G$, and let $\sigma: G \rightarrow \operatorname{Aut}_{\mathbb{C}}(V)$ be a finite-dimensional complex representation of $G$. (As always, we build continuity into the definition of "representation.") The Artin $L$ function is defined to be

$$
L(s, \sigma)=L(s, \sigma, K / F)=\prod_{\wp} L_{\wp}(s, \sigma),
$$

the product being taken over the nontrivial prime ideals $\wp$ in $\mathcal{O}_{F}$. Here $s$ is a complex variable.

Fix $\wp$, put $q=\left|\mathcal{O}_{F} / \wp\right|$, and let $P$ be a prime ideal in $\mathcal{O}_{K}$ lying over $\wp$. The definition of $L_{\wp}(s, \sigma)$ is a little simpler if $\wp$ is unramified in $K$, and we consider that case first. Then there is a well defined Frobenius element $\operatorname{Fr}_{P}$ in $G_{P}$, and we put $^{1}$

$$
L_{\wp}(s, \sigma)=\operatorname{det}\left(1-\sigma\left(\operatorname{Fr}_{P}\right) q^{-s}\right)^{-1} \quad \text { for } \operatorname{Re} s>0 .
$$

Let the eigenvalues of $\sigma\left(\operatorname{Fr}_{P}\right)$ be $\varepsilon_{1}, \ldots, \varepsilon_{\operatorname{dim} V}$; these are roots of unity since $\operatorname{Fr}_{P}$ has finite order. Then

$$
L_{\wp}(s, \sigma)=\prod_{i=1}^{\operatorname{dim} V}\left(1-\varepsilon_{i} q^{-s}\right)^{-1} .
$$

As $P$ varies, $\operatorname{Fr}_{P}$ moves in a conjugacy class of $G$. The eigenvalues of $\sigma\left(\operatorname{Fr}_{P}\right)$ do not change, and the second formula for $L_{\wp}(s, \sigma)$ shows that the function depends only on $\wp$.

Now suppose $\wp$ is allowed to be ramified in $K$, so that the inertia group $I_{P}$ is nontrivial. Let $V^{I_{P}}$ be the subspace of $V$ on which $\sigma\left(I_{P}\right)$ acts as the identity; this would be all of $V$ in the unramified case. Then $\sigma\left(G_{P}\right)$ preserves this space. If $\operatorname{Fr}_{P}$ is one lift to $G_{P}$ of the canonical generator of the Galois group of the residue field extension, then the most general lift is $\operatorname{Fr}_{P} i_{P}$ with $i_{P} \in I_{P}$. Thus $\sigma\left(\operatorname{Fr}_{P}\right)$ is unambiguous as a linear transformation on $V^{I_{P}}$, and we define ${ }^{2}$

$$
L_{\wp}(s, \sigma)=\operatorname{det}\left(1-\left.\sigma\left(\operatorname{Fr}_{P}\right)\right|_{V^{I_{P}}} q^{-s}\right)^{-1} \quad \text { for } \operatorname{Re} s>0 .
$$

Again we can rewrite this using eigenvalues, and we see that the result is independent of $P$.

Each Artin $L$ function converges for $\operatorname{Re} s>1$. Artin $L$ functions have the following additional properties (see [Hei, pp. 222-223], [La, pp. 236-239], and [Mar, p. 9]):

1) $L\left(s, \sigma_{1} \oplus \sigma_{2}, K / F\right)=L\left(s, \sigma_{1}, K / F\right) L\left(s, \sigma_{2}, K / F\right)$.

2) Suppose that $F \subset E \subset K$ and that $E$ is Galois over $F$. Let $H=\operatorname{Gal}(K / E)$, a normal subgroup of $G=\operatorname{Gal}(K / F)$. If $\tilde{\sigma}$ is a representation of $G$ lifted from a representation $\sigma$ of $G / H$, then

$$
L(s, \tilde{\sigma}, K / F)=L(s, \sigma, E / F) .
$$

Consequently an Artin $L$ function depends only on $s$ and a continuous finitedimensional representation of $\operatorname{Gal}(\bar{F} / F)$.

\footnotetext{
${ }^{1}$ Concerning the choice of $\operatorname{Fr}_{P}$ or its inverse in this formula (i.e., $\sigma$ or its contragredient), see Remark 1 after Corollary 2.5.

${ }^{2}$ See the footnote with (5.1a).
} 
3) Suppose that $F \subset E \subset K$ with $E / F$ possibly not Galois. Let $\sigma_{0}$ be a representation of $G_{0}=\operatorname{Gal}(K / E)$. Then

$$
L\left(s, \operatorname{ind}_{G_{0}}^{G}\left(\sigma_{0}\right), K / F\right)=L\left(s, \sigma_{0}, K / E\right) .
$$

A quasicharacter is a continuous homomorphism into $\mathbb{C}^{\times}$. Let $F$ be a number field. By a Grossencharacter of $F$ is meant a quasicharacter of $C_{F}$.

Example 1. Fix a positive integer $m$. A Dirichlet character modulo $m$ is the lift $\chi$ to $\mathbb{Z}$ of a character of the multiplicative group $(\mathbb{Z} / m \mathbb{Z})^{\times}$, with $\chi(a)$ set equal to 0 if $a$ and $m$ are not relatively prime. Fix such a $\chi$. With $F=\mathbb{Q}$, let $S$ consist of $\infty$ and the primes dividing $m$. In the notation of $\S 3$, define a homomorphism $\varphi: I^{S} \rightarrow S^{1}$ by $\varphi\left((a / b)^{S}\right)=\chi(a) / \chi(b)$ whenever $a$ and $b$ are integers relatively prime to $m$. If $p$ is a prime dividing $m$ and $p^{c}$ is the exact power of $p$ dividing $m$, then $\left|\frac{a}{b}-1\right|_{p}<p^{-c}$ implies that $a$ and $b$ are congruent modulo $p^{c}$. Hence if $\left|\frac{a}{b}-1\right|_{p}<m^{-1}$ for all $p$ dividing $m$, then $a$ and $b$ are congruent modulo $m$, and it follows that $\chi(a)=\chi(b)$. In other words $\varphi$ is admissible in the sense of $\S 3$. By Theorem 3.8 there exists a unique unitary Grossencharacter $\tilde{\varphi}$ of $\mathbb{Q}$ such that

$$
\tilde{\varphi}(1, \ldots, 1, p, 1, \ldots)=\chi(p)
$$

for all primes $p$ not dividing $m$; here $(1, \ldots, 1, p, 1, \ldots)$ denotes the idele that is $p$ in the $p^{\text {th }}$ place and is 1 elsewhere.

Example 2. If $\omega$ is any (continuous) character of $G_{F}^{\mathrm{ab}}$, then the composition $\omega \circ \theta_{F}$ with the Artin map of $F$ is a Grossencharacter. Since $\omega$ has to factor through a finite quotient of $G_{F}^{\mathrm{ab}}$, it is the same to consider compositions $\omega_{0} \circ \theta_{K / F}$, where $K$ is a finite abelian Galois extension of $F$ and $\omega_{0}$ is a character of $\operatorname{Gal}(K / F)$.

Let $\varphi$ be a Grossencharacter of $F$. Following Hecke in spirit, we shall associate an $L$ function $L(s, \varphi)$ to $\varphi$. For each finite place $v$ with corresponding prime ideal $\wp$ of $\mathcal{O}_{F}$, we can restrict $\varphi$ to the coordinate $F_{\wp}^{\times}$, obtaining a quasicharacter $\varphi_{\wp}: F_{\wp}^{\times} \rightarrow \mathbb{C}^{\times}$. Let $\mathcal{O}_{\wp}$ be the ring of integers in $F_{\wp}$. We say that $\varphi_{\wp}$ is ramified if $\left.\varphi_{\wp}\right|_{\mathcal{O}_{\wp}^{\times}}$is nontrivial. For each $\wp$, let $\varpi_{\wp}$ be a prime element in $\mathcal{O}_{\wp}$. Then the definition is ${ }^{3}$

$$
L(s, \varphi)=\prod_{\substack{\wp \text { unramified } \\ \text { for } \varphi}} L_{\wp}(s, \varphi)=\prod_{\substack{\text { unramified } \\ \text { for } \varphi}}\left(1-\frac{\varphi_{\wp}\left(\varpi_{\wp}\right)}{\left|\mathcal{O}_{F} / \wp\right|^{s}}\right)^{-1} .
$$

The functions $L(s, \varphi)$ have nice analytic properties. They have meromorphic continuations to $\mathbb{C}$ and satisfy a functional equation relating the values at $s$ and $1-s$. The only possible pole is at $s=1$ and is at most simple; there is no pole if $\varphi$ is nontrivial on $\left(\mathbb{A}_{F}^{\times}\right)^{1}$. These results are essentially due to Hecke. Later Tate [Ta1] found an important different proof that uses local-global methods. For an exposition of Tate's work and a higher-dimensional generalization, see Jacquet's lecture [Ja2].

Sometimes authors include in $L(s, \varphi)$ extra factors for the infinite places that involve a gamma function and powers of certain numbers. See [Kna2] for a description of these. Shortly we shall use this kind of completed $L$ function, writing $\Lambda$ for it. Inclusion of factors for the infinite places affects the poles of $L(s, \varphi)$ only slightly and makes the functional equation much simpler.

\footnotetext{
${ }^{3}$ See the footnote with (5.1a).
} 
Theorem 5.1. If $K / F$ is a finite abelian Galois extension of number fields and $\omega$ is a character of $\operatorname{Gal}(K / F)$, then the Artin $L$ function $L(s, \omega, K / F)$ equals the Hecke $L$ function $L(s, \varphi)$ of the Grossencharacter $\varphi=\omega \circ \theta_{K / F}$.

Proof. Let $H$ be the kernel of $\omega$ in $G=\operatorname{Gal}(K / F)$, and let $E$ be the fixed field of $H$ in $K$. Then $E / F$ is a finite abelian Galois extension of $F$, and $\omega$ descends to a one-one character $\omega_{0}$ of $G / H=\operatorname{Gal}(E / F)$. By property (2) of Artin $L$ functions, we have $L(s, \omega, K / F)=L\left(s, \omega_{0}, E / F\right)$. Also $\varphi=\omega_{0} \circ \varphi_{E / F}$. Thus it is enough to prove that $L\left(s, \omega_{0}, E / F\right)=L\left(s, \omega_{0} \circ \theta_{E / F}\right)$. We do so factor by factor.

Let $\wp$ be a nontrivial prime ideal in $\mathcal{O}_{F}$. We show that $\wp$ is unramified in $E$ if and only if $\wp$ is unramified for $\varphi$. With $S$ as the set of infinite places and places that ramify in $E$, first suppose that $\wp$ is not in $S$. If $x_{\wp}$ is in $\mathcal{O}_{\wp}^{\times}$, then the proof of Theorem 3.8 (with $a_{n}=1$ for all $n$ ) shows that

$$
\varphi_{\wp}\left(x_{\wp}\right)=\varphi\left(\ldots, 1, x_{\wp}, 1, \ldots\right)=\varphi\left(\left(\left\{x_{\wp}\right\}\right)^{S}\right)=\omega_{0}\left[\frac{E / F}{(1)}\right]=1 .
$$

Hence $\wp$ is unramified for $\varphi$.

Conversely suppose $\wp$ is unramified for $\varphi=\omega_{0} \circ \theta_{E / F}$. This means that $\varphi_{\wp}=$ $\omega_{0} \circ \theta_{E / F} \circ i_{\wp}$ is 1 on $\mathcal{O}_{\wp}^{\times}$. By Theorem 4.5, $\omega_{0} \circ \theta_{E_{P} / F_{\wp}}$ is 1 on $\mathcal{O}_{\wp}^{\times}$. Since $\omega_{0}$ is one-one, $\theta_{E_{P} / F_{\wp}}$ is 1 on $\mathcal{O}_{\wp}^{\times}$. Referring to $(2.3)$ and Corollary 2.5 , we see that the inertia group $I_{P}$ is trivial. Thus $\wp$ is not in $S$.

Now suppose that $\wp$ is ramified (in both senses). Then the $\wp$ factor of $L(s, \varphi)$ is 1 by definition. Meanwhile the $\wp$ factor of $L\left(s, \omega_{0}, E / F\right)$ is $\left(1-\left.\omega_{0}\left(\operatorname{Fr}_{P}\right)\right|_{\mathbb{C}^{I_{P}}} q^{-s}\right)^{-1}$. Since $\omega_{0}$ is one-one and $I_{P}$ is nontrivial, $\mathbb{C}^{I_{P}}=0$. Thus the $\wp$ factor for each $L$ function is 1 in the ramified case.

Finally suppose that $\wp$ is unramified (in both senses). Theorem 4.5 gives

$$
\varphi_{\wp}=\varphi \circ i_{\wp}=\omega \circ \theta_{K / F} \circ i_{\wp}=\omega_{0} \circ \theta_{E / F} \circ i_{\wp}=\omega_{0} \circ \theta_{E_{P} / F_{\wp}} .
$$

Then

$$
L_{\wp}\left(s, \omega_{0}, E / F\right)=\left(1-\omega_{0}\left(\operatorname{Fr}_{P}\right) q^{-s}\right)^{-1}
$$

by definition, while

$$
\begin{aligned}
L_{\wp}(s, \varphi) & =\left(1-\varphi_{\wp}\left(\varpi_{\wp}\right) q^{-s}\right)^{-1} & & \text { by definition } \\
& =\left(1-\omega_{0} \theta_{E_{P} / F_{\wp}}\left(\varpi_{\wp}\right) q^{-s}\right)^{-1} & & \text { by }(5.3) \\
& =\left(1-\omega_{0}\left(\operatorname{Fr}_{P}\right) q^{-s}\right)^{-1} & & \text { by Theorem 2.2. }
\end{aligned}
$$

Hence the $\wp$ factors for the two $L$ functions match in the unramified case.

Corollary 5.2. If $K / F$ is a finite abelian Galois extension of number fields and $\omega$ is a character of $\operatorname{Gal}(K / F)$, then $L(s, \omega, K / F)$ extends to be entire in $\mathbb{C}$. For the trivial character $\omega=1, L(s, 1, K / F)$ extends to be meromorphic in $\mathbb{C}$ with a simple pole at $s=1$.

Of course, it is immediate from the properties of Artin $L$ functions that many more such functions are entire. For example, let $K / F$ be any finite Galois extension. Then the Artin $L$ function for a representation of $\operatorname{Gal}(K / F)$ induced from a nontrivial one-dimensional representation is entire. Thus for any monomial group, i.e., any finite group whose irreducible representations are all induced from onedimensional representations of subgroups, the Artin $L$ function of any nontrivial irreducible representation is entire. Dihedral groups are examples of monomial groups. 
Artin Conjecture. Let $K / F$ be any finite Galois extension of number fields, and let $\sigma$ be a nontrivial irreducible representation of $\operatorname{Gal}(K / F)$. Then $L(s, \sigma, K / F)$ extends to be entire in $\mathbb{C}$.

The Artin $L$ function, which we defined to include only factors (5.1) from the finite places, has a natural completion by adjoining some gamma factors for the infinite places (see $[\mathrm{Kna} 2,(3.6)$ and (4.6)], and we shall denote the completed $L$ function by $\Lambda(s, \sigma, K / F)$. It is actually conjectured that $\Lambda(s, \sigma, K / F)$ is entire if $\sigma$ is irreducible and nontrivial.

Despite the fact that the Artin Conjecture is not known, Brauer's Induction Theorem says that an Artin $L$ function has a continuation to all of $\mathbb{C}$ that is at least meromorphic and that the continued function satisfies the same kind of functional equation as a Hecke $L$ function. A more detailed statement of Brauer's result is as follows.

Theorem 5.3. The group character of any complex finite-dimensional representation of a finite group is an integer combination of group characters of representations induced from one-dimensional representations of subgroups. Consequently any Artin $L$ function has a continuation to all of $\mathbb{C}$ that is at least meromorphic. Moreover each Artin L function satisfies a functional equation of the form

$$
\Lambda(s, \sigma, K / F)=\varepsilon(s, \sigma, K / F) \Lambda\left(1-s, \sigma^{\vee}, K / F\right),
$$

where $\sigma^{\vee}$ is the contragredient and $\varepsilon(s, \sigma, K / F)$ is entire and nonvanishing.

The Langlands theory proposes addressing the Artin Conjecture by introducing $L$ functions that generalize those of Hecke and by showing that Artin $L$ functions are always of this kind. Aspects of this theory occupy much of the remainder of this article. For another exposition of this kind, see [Gelb2].

\section{Linear Reductive Algebraic Groups}

Let $F$ be a number field. Informally a linear algebraic group of $n$-by- $n$ matrices over $F$ is a group $G$ of $n$-by- $n$ matrices defined by polynomial equations in $n^{2}$ matrix variables with coefficients in $F$. For a precise definition, see [Bo2], [Bo3], or [We1].

Clearing fractions in the defining equations of $G$, we may assume that the coefficients are all in $\mathcal{O}_{F}$. If $R$ is a torsion-free commutative ring containing $\mathcal{O}_{F}$ as a subring, then the group of $R$ points of $G$ is well defined, independently of how we cleared fractions, and we denote this group by $G(R)$. The group $G$ will be said to be unipotent if $G(\mathbb{C})$ consists entirely of unipotent matrices, while $G$ is reductive if $\{1\}$ is the only connected unipotent normal subgroup of $G(\mathbb{C})$. The reader may wish to think of a reductive $G$ as being $G L_{n}$ or $S L_{n}$, and little will be lost for current purposes by doing so.

A simple example of a linear algebraic group is the affine line, whose $R$ points are the elements of $R$. To work with this algebraic group as a linear group, we may view it as the group of all matrices $\left(\begin{array}{ll}1 & * \\ 0 & 1\end{array}\right)$. Another example is the multiplicative group, whose $R$ points are the elements of $R^{\times}$; this group is also called $G L_{1}$. The affine line is unipotent, and the multiplicative group is reductive.

If $v$ is a place of $F$, we can topologize $G L_{n}\left(F_{v}\right)$ as an open subset of $n^{2}$ dimensional space, and the result is a locally compact group. Then $G\left(F_{v}\right)$ is given 
the relative topology from $G L_{n}\left(F_{v}\right)$ and is a locally compact group. If $v$ is finite, then $G\left(\mathcal{O}_{v}\right)$ is a compact subgroup of $G\left(F_{v}\right)$.

For each finite set $S$ of places containing the set $S_{\infty}$ of infinite places, the group $G\left(\mathbb{A}_{F}(S)\right)$ is well defined since the ring $\mathbb{A}_{F}(S)$ contains diagonally embedded $\mathcal{O}_{F}$. The group $G\left(\mathbb{A}_{F}(S)\right)$ is nothing more than the direct product of all $G\left(F_{v}\right)$ for $v \in S$ and all $G\left(\mathcal{O}_{v}\right)$ for $v \notin S$. It is locally compact. Its topology may be described alternatively as the relative topology from $G L_{n}\left(\mathbb{A}_{F}(S)\right)$.

Similarly, the group $G\left(\mathbb{A}_{F}\right)$ of $\mathbb{A}_{F}$ points of $G$ is well defined since $\mathbb{A}_{F}$ contains diagonally embedded $F$. When $G$ is the affine line, $G\left(\mathbb{A}_{F}\right)$ is just the group of adeles of $F$; when $G$ is the multiplicative group, $G\left(\mathbb{A}_{F}\right)$ is the group of ideles of $F$. In every case, a member of $G\left(\mathbb{A}_{F}\right)$ may be regarded as a tuple of matrices indexed by the places of $F$, the matrix in the $v^{\text {th }}$ place being in $G\left(F_{v}\right)$, with almost all such matrices lying in $G\left(\mathcal{O}_{v}\right)$.

As is the case with the ideles, the topology on $G\left(\mathbb{A}_{F}\right)$ is not necessarily the relative topology from $n$-by- $n$ matrices over $\mathbb{A}_{F}$. Instead the topology is the restricted direct product topology of the $G\left(F_{v}\right)$ relative to the $G\left(\mathcal{O}_{v}\right)$. In other words, it is the direct limit topology from the subgroups $G\left(\mathbb{A}_{F}(S)\right)$, which are to be open. Alternatively we can topologize $G\left(\mathbb{A}_{F}\right)$ by embedding it in matrices $\left(\begin{array}{cc}x & 0 \\ 0(\operatorname{det} x)^{-1}\end{array}\right)$ of size $n+1$ and giving it the relative topology from $(n+1)^{2}$-dimensional space over $\mathbb{A}_{F}$. With these definitions, $G\left(\mathbb{A}_{F}\right)$ gets the relative topology from $G L_{n}\left(\mathbb{A}_{F}\right)$.

Because of this second way of realizing the topology, it follows that the group $G$ (diagonally embedded $F$ ), which equals diagonally embedded $G(F)$, is a discrete subgroup of $G\left(\mathbb{A}_{F}\right)$. We write $G(F)$ for this subgroup. The first theorem generalizes Corollary 3.6.

Theorem 6.1. If $G$ is a reductive linear algebraic group, then the number of double cosets in $G(F) \backslash G\left(\mathbb{A}_{F}\right) / G\left(\mathbb{A}_{F}\left(S_{\infty}\right)\right)$ is finite. For $G=G L_{n}$, the number of double cosets is the class number of $F$. For $G=S L_{n}$, the number of double cosets is 1 .

REFERENCES.

1) In a classical setting with $G(\mathbb{Z}) \subset G(\mathbb{Q})$, this theorem is due to Borel and Harish-Chandra [Bo-HC]. The result in the classical setting says in part that if $G$ is a semisimple linear algebraic group over $\mathbb{Q}$, then $G(\mathbb{Z}) \backslash G(\mathbb{R})$ has finite volume. The result in an adelic setting appears in Borel [Bo1, p. 19]. The relationship between the two settings will be described below.

2 ) If the group $G\left(\mathbb{A}_{F}\left(S_{\infty}\right)\right)$ in the statement of the theorem is replaced by an open subgroup of finite index, then it is clear that the number of double cosets remains finite.

More is true than is asserted in the theorem. The hypothesis that $G$ is reductive is unnecessary. In any event, let $G_{\infty}=G\left(F_{\infty}\right)$ be the archimedean component of $G\left(\mathbb{A}_{F}\right)$ (equal to 1 at every finite place). We say that $G$ has the strong approximation property if $G(F) G_{\infty}$ is dense in $G\left(\mathbb{A}_{F}\right)$. In this case the number of double cosets in $G(F) \backslash G\left(\mathbb{A}_{F}\right) / G\left(\mathbb{A}_{F}\left(S_{\infty}\right)\right)$ is automatically 1 . The (unipotent) affine line has the strong approximation property [Cas, p. 67], and so does the reductive $S L_{n}$. For more discussion of this property, see [Kne].

In the classical setting for automorphic forms, one works with the quotient $G(\mathbb{Z}) \backslash G(\mathbb{R})$. Following [Bo-Ja, p. 195], let us see how this quotient space is related to the double coset decomposition in Theorem 6.1. We decompose $G\left(\mathbb{A}_{F}\left(S_{\infty}\right)\right)=$ 
$G_{\infty} \times K_{1}$, where $G_{\infty}=G\left(F_{\infty}\right)$ is the archimedean component and where $K_{1}=$ $G\left(U_{1}\right)=G\left(\prod_{v}\right.$ finite $\left.\mathcal{O}_{v}\right)$ is the nonarchimedean component (equal to 1 at every infinite place). Let $K$ be any open subgroup of finite index in $K_{1}$, and use Theorem 6.1 to write

$$
G\left(\mathbb{A}_{F}\right)=\coprod_{c \in C} G(F) c G_{\infty} K
$$

as a disjoint union, for some finite subset $C$ of $G\left(\mathbb{A}_{F}\right)$. Without loss of generality, we may assume that the members of $C$ all have component 1 at the infinite place. For $c \in C$, define $G_{c}=G_{\infty} c K c^{-1}$ and $\Gamma_{c}=G_{c} \cap G(F)$. The group $G_{c}$ is open in $G\left(\mathbb{A}_{F}\right)$, and the discreteness of $G(F)$ in $G\left(\mathbb{A}_{F}\right)$ implies that $\Gamma_{c}$ is a discrete subgroup of $G_{c}$. Since $c K c^{-1}$ is compact, we may use projection on the infinite places to identify $\Gamma_{c}$ with a discrete subgroup of $G_{\infty}$. If $f$ is a right $K$ invariant function on $G\left(\mathbb{A}_{F}\right)$ and if $c$ is in $C$, let $f_{c}$ be the function $x \mapsto f(c x)$ on $G_{\infty}$. Then we readily check that the map

$$
f \mapsto\left\{f_{c}\right\}_{c \in C}
$$

is a bijection of the space of functions on $G(F) \backslash G\left(\mathbb{A}_{F}\right) / K$ with the space of functions on $\coprod_{c \in C}\left(\Gamma_{c} \backslash G_{\infty}\right)$. Thus we obtain an identification

$$
G(F) \backslash G\left(\mathbb{A}_{F}\right) / K=\coprod_{c \in C}\left(\Gamma_{c} \backslash G_{\infty}\right) .
$$

Formula (6.3) is especially simple in cases where $C=\{1\}$. Examples, all with $K=K_{1}$, are when $G$ is the affine line and $F$ is arbitrary, when $G=S L_{n}$ and $F$ is arbitrary, and when $G=G L_{n}$ and $F=\mathbb{Q}$. For these examples, (6.3) becomes

and

$$
F \backslash \mathbb{A}_{F} / \prod_{\wp} \mathcal{O}_{F_{\wp}}=\mathcal{O}_{F} \backslash F_{\infty},
$$

$$
\begin{gathered}
S L_{n}(F) \backslash S L_{n}\left(\mathbb{A}_{F}\right) / \prod_{\wp} S L_{n}\left(\mathcal{O}_{F_{\wp}}\right)=S L_{n}\left(\mathcal{O}_{F}\right) \backslash S L_{n}\left(F_{\infty}\right) \\
G L_{n}(\mathbb{Q}) \backslash G L_{n}\left(\mathbb{A}_{\mathbb{Q}}\right) / \prod_{p} G L_{n}\left(\mathbb{Z}_{p}\right)=G L_{n}(\mathbb{Z}) \backslash G L_{n}(\mathbb{R}) .
\end{gathered}
$$

The right side of (6.3) is more concrete than the left side, but part of the action is lost in working with the right side rather than with the adeles. For instance, in the adelic picture of $(6.4 \mathrm{c})$, each of the groups $G L_{n}\left(\mathbb{Q}_{p}\right)$ acts on $G L_{n}(\mathbb{Q}) \backslash G L_{n}\left(\mathbb{A}_{\mathbb{Q}}\right)$, and the corresponding action on this space by functions on $G L_{n}\left(\mathbb{Q}_{p}\right)$ biinvariant under $G L_{n}\left(\mathbb{Z}_{p}\right)$ descends to an action on the left side of (6.4c). This action is hidden, however, in the realization as $G L_{n}(\mathbb{Z}) \backslash G L_{n}(\mathbb{R})$.

Let us describe the functor "restriction of the ground field," which has the property of reducing aspects of the theory over the number field $F$ to the theory over $\mathbb{Q}$. We follow $\left[\right.$ We1]. Let $d=[F: \mathbb{Q}]$, and let $\sigma_{1}, \ldots, \sigma_{d}$ be the distinct field maps of $F$ into $\overline{\mathbb{Q}}$ fixing $\mathbb{Q}$. Let $V$ be an affine variety over $F$. A pair $(W, p)$, in which $W$ is an affine variety over $\mathbb{Q}$ and $p: W \rightarrow V$ is an algebraic map defined over $F$, is said to be a variety obtained from $V$ by restriction of the ground field from $F$ to $\mathbb{Q}$ if the map

$$
\left(p^{\sigma_{1}}, \ldots, p^{\sigma_{d}}\right): W \rightarrow \prod_{j=1}^{d} V^{\sigma_{j}},
$$


which is defined over $\overline{\mathbb{Q}}$, is an isomorphism of varieties. Such a pair $(W, p)$ exists and is unique up to a canonical isomorphism over $\mathbb{Q}$. It is customary to drop the $p$ from the notation and write $W=R_{F / \mathbb{Q}} V$, regarding $R_{F / \mathbb{Q}}$ as a functor from affine varieties over $F$ to affine varieties over $\mathbb{Q}$. Restriction of the ground field has the key property that $\left(R_{F / \mathbb{Q}} V\right)(A)=V\left(F \otimes_{\mathbb{Q}} A\right)$ for any $\mathbb{Q}$ algebra $A$.

\section{Examples.}

1) If $V$ is the affine line over $F$, then $W$ is the vector space $V$ regarded as a $\mathbb{Q}$ vector space of dimension $d$. To put this example in the above context, let $\left\{\alpha_{j}\right\}_{j=1}^{d}$ be a basis of $F$ over $\mathbb{Q}$, and define $p\left(x_{1}, \ldots, x_{d}\right)=\sum_{j} \alpha_{j} x_{j}$. Then $p^{\sigma}\left(x_{1}, \ldots, x_{d}\right)=$ $\sum_{j} \alpha_{j}^{\sigma} x_{j}$, and

$$
\left(p^{\sigma_{1}}, \ldots, p^{\sigma_{d}}\right)\left(x_{1}, \ldots, x_{d}\right)=\left(\sum_{j} \alpha_{j}^{\sigma_{1}} x_{1}, \ldots, \sum_{j} \alpha_{j}^{\sigma_{d}} x_{j}\right) .
$$

The fact that $\left(p^{\sigma_{1}}, \ldots, p^{\sigma_{d}}\right)$ is an isomorphism follows from the fact that $\operatorname{det}\left\{\alpha_{j}^{\sigma_{i}}\right\} \neq$ 0 .

2) The previous example may be extended to an $n$-dimensional affine space $V$ over $F$ in obvious fashion, with

$$
p\left(x_{11}, \ldots, x_{1 d}, x_{21}, \ldots, x_{n d}\right)=\left(\sum_{j=1}^{d} \alpha_{j} x_{1 j}, \ldots, \sum_{j=1}^{d} \alpha_{j} x_{n j}\right) .
$$

More generally if $V$ is the variety in $n$-dimensional affine space defined by some polynomials $P\left(X_{1}, \ldots, X_{n}\right)$, we can define a variety $W$ in the $n d$-dimensional affine space $W$ by rewriting

$$
P\left(\sum_{j=1}^{d} \alpha_{j} x_{1 j}, \ldots, \sum_{j=1}^{d} \alpha_{j} x_{n j}\right)=\alpha_{1} p_{1}\left(x_{11}, \ldots, x_{n d}\right)+\cdots+\alpha_{d} p_{d}\left(x_{11}, \ldots, x_{n d}\right)
$$

and replacing $P$ by $p_{1}, \ldots, p_{d}$.

Restriction of the ground field is a functor of linear algebraic groups. Specifically if $V=G$ is a linear algebraic group over $F$, then the pair $\left(R_{F / \mathbb{Q}}, p\right)$ can be taken to consist of a linear algebraic group over $\mathbb{Q}$ and a homomorphism over $F$, and the linear algebraic group structure is unique up to a canonical isomorphism of algebraic groups. The map $\left(p^{\sigma_{1}}, \ldots, p^{\sigma_{d}}\right): R_{F / \mathbb{Q}} G \rightarrow \prod_{j=1}^{d} G^{\sigma_{j}}$ is then an isomorphism over $\overline{\mathbb{Q}}$ of algebraic groups. Because of the formula $\left(R_{F / \mathbb{Q}} G\right)(A)=G\left(F \otimes_{\mathbb{Q}} A\right)$, the isomorphism $\left(p^{\sigma_{1}}, \ldots, p^{\sigma_{d}}\right)$ induces isomorphisms

$$
\begin{aligned}
\left(R_{F / \mathbb{Q}} G\right)(\overline{\mathbb{Q}}) & \cong G(\bar{Q}) \times \cdots \times G(\bar{Q}), \\
\left(R_{F / \mathbb{Q}} G\right)(\mathbb{Q}) & \cong G(F), \\
\left(R_{F / \mathbb{Q}} G\right)(\mathbb{R}) & \cong G_{\infty}, \\
\left(R_{F / \mathbb{Q}} G\right)\left(\mathbb{A}_{\mathbb{Q}}\right) & \cong G\left(\mathbb{A}_{F}\right) .
\end{aligned}
$$

Similarly we can define $R_{E / F} G$ as a reductive group over $F$ whenever $E / F$ is an extension of number fields and $G$ is a reductive group over $E$.

With $G$ defined over $F$, let $X^{*}(G)_{F}$ be the set of all $F$ rational homomorphisms of $G$ into $G L_{1}$. If $\chi$ is in $X^{*}(G)_{F}$, then $\chi$ extends at each place to a continuous homomorphism $\chi_{v}: G\left(F_{v}\right) \rightarrow F_{v}^{\times}$. Let $\chi_{\mathbb{A}_{F}}: G\left(\mathbb{A}_{F}\right) \rightarrow \mathbb{A}_{F}^{\times}$be the product of 
the $\chi_{v}$. Then $\left|\chi_{\mathbb{A}_{F}}\right|_{\mathbb{A}_{F}}$ is a homomorphism of $G\left(\mathbb{A}_{F}\right)$ into $\mathbb{R}_{+}^{\times}$. Define $G\left(\mathbb{A}_{F}\right)^{1}=$ $\bigcap_{\chi \in X^{*}(G)_{F}} \operatorname{ker}\left|\chi_{\mathbb{A}_{F}}\right|_{\mathbb{A}_{F}}$.

For example, if $G=G L_{n}$, then $X^{*}(G)_{F}$ consists of the integral powers of the determinant, and $\left|\operatorname{det}_{\mathbb{A}_{F}}\left(g_{v}\right)\right|_{\mathbb{A}_{F}}=\prod_{v}\left|\operatorname{det} g_{v}\right|_{v}$. Hence $G\left(\mathbb{A}_{F}\right)^{1}$ consists of the $n$ by- $n$ matrices over $\mathbb{A}_{F}$ for which the module of the determinant is 1 . In the special case that $n=1$, this reduces to the group $\left(\mathbb{A}_{F}^{\times}\right)^{1}$ that appears in Theorem 3.5. The following theorem generalizes Theorem 3.5.

Theorem 6.2. Suppose that $G(\mathbb{C})$ is connected. Then the group $G(F)$ lies in $G\left(\mathbb{A}_{F}\right)^{1}$, and the quotient space $G(F) \backslash G\left(\mathbb{A}_{F}\right)^{1}$ has finite volume. Moreover, $G(F) \backslash G\left(\mathbb{A}_{F}\right)^{1}$ is compact if and only if every unipotent element of $G(F)$ belongs to the radical of $G(F)$.

References. [Bo-HC] and [Bo1, p. 22].

An example of a nonabelian $G$ for which compactness of $G(F) \backslash G\left(\mathbb{A}_{F}\right)^{1}$ follows from this theorem is the multiplicative group $D^{\times}$of a finite-dimensional division algebra $D$ of $F$ with center $F$. For a direct proof of the compactness in this case, see [Gf-Gr-P] or [We4, p. 74]. To prove the compactness from Theorem 6.2, we note that a unipotent element of $G(F)$ is of the form $1+x$ with $x$ nilpotent. Since $D$ is closed under addition, $x$ must be in $D(F)$. Since $x$ is nilpotent, $x$ is not invertible. Thus $x=0$, and the theorem applies.

\section{Automorphic Forms and Automorphic Representations}

Historically the theory of automorphic forms began with modular and cusp forms for the group $S L_{2}(\mathbb{Z})$. For a detailed discussion of such forms, see [Kna1] or [Shi]. Briefly if $g=\left(\begin{array}{ll}a & b \\ c & d\end{array}\right)$ is in $G L(2, \mathbb{R})$, let $g(z)=\frac{a z+b}{c z+d}$ for all nonreal complex numbers $z$, and define

$$
j(g, z)=(c z+d)(\operatorname{det} g)^{1 / 2} .
$$

In our discussion, $j(g, z)$ will occur only in the form $j(g, z)^{2}$, and we may consequently use either determination of the square root of $(\operatorname{det} g)^{1 / 2}$.

A modular form of weight $k$ (an even integer) for $S L_{2}(\mathbb{Z})$ is an analytic function $f(z)$ on the upper half plane such that

$$
f(\gamma(z))=j(\gamma, z)^{k} f(z) \quad \text { for all } \gamma \in S L_{2}(\mathbb{Z})
$$

and such that $f$ is analytic at $\infty$ in the following sense: The function $f$, being analytic and periodic under $z \mapsto z+1$, has an expansion $f(z)=\sum_{n=-\infty}^{\infty} c_{n} e^{2 \pi i n z}$, and the condition of analyticity at $\infty$ is that $c_{n}=0$ for $n<0$. The condition at $\infty$ for a modular form can alternatively be formulated as the slow-growth condition

$$
|f(x+i y)| \leq C y^{N} \quad \text { for some } C \text { and } N \text { as } y \rightarrow+\infty .
$$

A cusp form is a modular form that vanishes at $\infty$ in the sense that $c_{0}=0$. The additional condition that a modular form is a cusp form can be reformulated as the vanishing of an integral:

$$
\int_{0}^{1} f(x+i y) d x=0 \quad \text { for some or equivalently every } y>0 .
$$


A cusp form satisfies the rapid-decrease condition that for each $N$

$$
|f(x+i y)| \leq C y^{-N} \quad \text { for some } C \text { as } y \rightarrow+\infty .
$$

A cusp form of weight $k$ can be expanded as $f(z)=\sum_{n=1}^{\infty} c_{n} e^{2 \pi i n z}$, and the associated $L$ function of $f$, defined by $L(s, f)=\sum_{n=1}^{\infty} c_{n} / n^{s}$, satisfies a functional equation relating the values at $s$ and $k-s$. Hecke introduced what we now call Hecke operators on the space of cusp forms of weight $k$. A computation using contour integrals shows that this space is finite-dimensional. The Hecke operators commute and are simultaneously diagonable. The eigenfunctions all have $c_{1} \neq 0$, and if $c_{1}$ is normalized to be 1 for an eigenfunction, then the corresponding $L$ function has an Euler product expansion, the product being taken over all primes.

Gelfand and Fomin were the first to notice that cusp forms could be realized as smooth vectors in representations of a certain ambient Lie group. It is possible to arrange for modular and cusp forms to lift both to $S L_{2}(\mathbb{Z}) \backslash S L_{2}(\mathbb{R})$ and to $G L_{2}(\mathbb{Z}) \backslash G L_{2}(\mathbb{R})$, and we shall indicate both liftings.

The details for the lifting to $S L_{2}(\mathbb{Z}) \backslash S L_{2}(\mathbb{R})$ are in [Gelb1] and also [Bump]. Given a modular form $f$ as above, we define

$$
\phi_{f, \infty}(g)=f(g(i)) j(g, i)^{-k}
$$

for $g \in S L_{2}(\mathbb{R})$. Then $\phi_{\infty}=\phi_{f, \infty}$ has the properties that

(i) $\phi_{\infty}(\gamma g)=\phi_{\infty}(g)$ for all $\gamma \in S L_{2}(\mathbb{Z})$

(ii) $\phi_{\infty}(g r(\theta))=e^{-i k \theta} \phi_{\infty}(g)$ for all $r(\theta)=\left(\begin{array}{rr}\cos \theta & -\sin \theta \\ \sin \theta & \cos \theta\end{array}\right)$

(iii) $\phi_{\infty}(g)$ satisfies the slow-growth condition that

$$
\left|\phi_{\infty}\left(\left(\begin{array}{ll}
1 & x \\
0 & 1
\end{array}\right)\left(\begin{array}{cc}
y^{1 / 2} & 0 \\
0 & y^{-1 / 2}
\end{array}\right) r(\theta)\right)\right| \leq C y^{N} \quad \text { for some } C \text { and } N \text { as } y \rightarrow+\infty .
$$

(iv) $\Omega \phi_{\infty}=-\frac{k}{2}\left(\frac{k}{2}-1\right) \phi_{\infty}$ for a suitable normalization of the Casimir operator $\Omega$ of $S L_{2}(\mathbb{R})$

(v) if $f$ is a cusp form, then $\phi_{\infty}$ is cuspidal in the sense that

$$
\int_{0}^{1} \phi_{\infty}\left(\left(\begin{array}{ll}
1 & x \\
0 & 1
\end{array}\right) g\right) d x=0 \quad \text { for all } g .
$$

For the lifting to $G L_{2}(\mathbb{Z}) \backslash G L_{2}(\mathbb{R})$, we start by extending $f$ to all nonreal complex numbers by putting $f(-z)=f(z)$. Then we define $\phi_{f, \infty}(g)$ by $(7.1)$ for $g \in G L_{2}(\mathbb{R})$. The invariance property in (i) extends to be valid for $\gamma \in G L_{2}(\mathbb{Z})$, properties (ii) through (v) are unchanged, and there is one new property:

(vi) $\phi_{\infty}(z g)=\phi_{\infty}(g)$ for all $g$ in the center of $G L_{2}(\mathbb{R})$.

It is natural to expect at first that $S L_{2}(\mathbb{R})$ is the better group to use for the above lifting, but it has become customary to lift to $G L_{2}(\mathbb{R})$ or the positive-determinant subgroup rather than $S L_{2}(\mathbb{R})$ in order to be able to incorporate Hecke operators more conveniently into the theory.

There are some other classical theories of automorphic forms that can be lifted to Lie groups in the same way. The theory of Maass forms [Maa] concerns certain nonholomorphic functions on the upper half plane, and these lift to $G L_{2}(\mathbb{Z}) \backslash G L_{2}(\mathbb{R})$. 
A theory [We3] begun by Hecke for analytic functions on the upper half plane transforming under the group

$$
\Gamma_{0}(N)=\left\{\left(\begin{array}{ll}
a & b \\
c & d
\end{array}\right) \in S L_{2}(\mathbb{Z}) \mid N \text { divides } c\right\}
$$

leads to functions on $G L_{2}(\mathbb{R})$ invariant under the group generated by $\Gamma_{0}(N)$ and $\left(\begin{array}{rr}1 & 0 \\ 0 & -1\end{array}\right)$. In addition, the theory of Hilbert modular forms [Ga] leads to quotients of products of several copies of $G L_{2}(\mathbb{R})$, and the theory of Siegel modular forms [Si] leads to quotients of real symplectic groups.

In each case the theory can be reinterpreted in an adelic setting by means of (6.3). For example, with classical modular and cusp forms with respect to $S L_{2}(\mathbb{Z})$, the isomorphism (6.4c) tells us that $f$ should be lifted to

$$
\phi_{f}(g)=f\left(g_{\infty}(i)\right) j\left(g_{\infty}, i\right)^{-k} \quad \text { for } g \in G L_{2}\left(\mathbb{A}_{\mathbb{Q}}\right)
$$

if $g=\gamma g_{\infty} k_{1}$ is the decomposition of $g \in G L_{2}\left(\mathbb{A}_{\mathbb{Q}}\right)$ according to $(6.4 \mathrm{c})$ reformulated as

$$
G L_{2}\left(\mathbb{A}_{\mathbb{Q}}\right)=G L_{2}(\mathbb{Q}) G L_{2}(\mathbb{R}) \prod_{p} G L_{n}\left(\mathbb{Z}_{p}\right) .
$$

The function $\phi=\phi_{f}$ on $G L_{2}\left(\mathbb{A}_{\mathbb{Q}}\right)$ has the properties that

(i) $\phi(\gamma g)=\phi(g)$ for all $\gamma \in G L_{2}(\mathbb{Q})$

(ii) $\phi\left(g k_{1}\right)=\phi(g)$ for all $k_{1} \in \prod_{p} G L_{2}\left(\mathbb{Z}_{p}\right)$

(iii) $\phi\left(g r_{\infty}(\theta)\right)=e^{-i k \theta} \phi(g)$ for all $r_{\infty}(\theta)=\left(\begin{array}{rr}\cos \theta & -\sin \theta \\ \sin \theta & \cos \theta\end{array}\right)$ at the infinite place

(iv) as a function of the variable in the infinite place, $\phi$ satisfies the equation $\Omega \phi=-\frac{k}{2}\left(\frac{k}{2}-1\right) \phi$ for a suitable normalization of the Casimir operator $\Omega$ of $S L_{2}(\mathbb{R})$

(v) $\phi(z g)=\phi(g)$ for all scalar $z$ in $G L_{2}\left(\mathbb{A}_{\mathbb{Q}}\right)$

(vi) $\phi(g)$ satisfies the following slow-growth condition: for each $c>0$ and compact subset $\omega$ of $G L_{2}\left(\mathbb{A}_{\mathbb{Q}}\right)$, there exist constants $C$ and $N$ such that

$$
\phi\left(\left(\begin{array}{cc}
a & 0 \\
0 & 1
\end{array}\right) g\right) \leq C|a|^{N}
$$

for all $g \in \omega$ and $a \in \mathbb{A}_{\mathbb{Q}}^{\times}$with $|a|_{\mathbb{A}_{\mathbb{Q}}}>c$

(vii) if $f$ is a cusp form, then $\phi$ is cuspidal in the sense that

$$
\int_{\mathbb{Q} \backslash \mathbb{A}_{\mathbb{Q}}} \phi\left(\left(\begin{array}{ll}
1 & x \\
0 & 1
\end{array}\right) g\right) d x=0 \quad \text { for all } g \in G L_{2}\left(\mathbb{A}_{\mathbb{Q}}\right) .
$$

The group $S L_{2}(\mathbb{Z})$, relative to which $f$ satisfies an invariance property, is captured by the compact group in (ii). The relevant identity is

$$
G L_{2}(\mathbb{Q}) \cap\left(G L_{2}(\mathbb{R}) \times \prod_{p} G L_{2}\left(\mathbb{Z}_{p}\right)\right)=G L_{2}(\mathbb{Z}) .
$$

For Hecke's theory with $\Gamma_{0}(N)$, the corresponding compact group that appears in (ii) is $\prod_{p} K_{p}^{\prime}$, where

$$
K_{p}^{\prime}=\left\{\left(\begin{array}{ll}
a & b \\
c & d
\end{array}\right) \in G L_{2}\left(\mathbb{Z}_{p}\right) \mid c / N \in \mathbb{Z}_{p}\right\} .
$$


The group $K_{p}^{\prime}$ coincides with $G L_{2}\left(\mathbb{Z}_{p}\right)$ for all $p$ prime to $N$, and the relevant identity is

$$
G L_{2}(\mathbb{Q}) \cap\left(G L_{2}(\mathbb{R}) \times \prod_{p} K_{p}^{\prime}\right)=\Gamma_{0}(N) \cup\left(\begin{array}{cc}
1 & 0 \\
0 & -1
\end{array}\right) \Gamma_{0}(N) .
$$

This adelic setting is what we generalize to arbitrary reductive groups, following [Bo-Ja]. Let $F$ be a number field, let $\mathbb{A}=\mathbb{A}_{F}$ be the adeles of $F$, and let $G$ be a reductive group over $F$ such that $G(\mathbb{C})$ is connected. Let $Z$ be a maximal $F$ split torus of the center of $G$. Let $G_{\infty}=G\left(F_{\infty}\right)$ be the archimedean component of $G(\mathbb{A})$, and let $G\left(\mathbb{A}_{f}\right)$ be the nonarchimedean component, so that

$$
G(\mathbb{A})=G_{\infty} \times G\left(\mathbb{A}_{f}\right) .
$$

Let

$$
\begin{aligned}
K_{\infty} & =\text { a maximal compact subgroup of the Lie group } G_{\infty} \\
\mathfrak{g} & =\text { complexification of the (real) Lie algebra of } G_{\infty} \\
U(\mathfrak{g}) & =\text { universal enveloping algebra of } \mathfrak{g} \\
Z(\mathfrak{g}) & =\text { center of } U(\mathfrak{g}) .
\end{aligned}
$$

Let $K_{1}$ be the open compact subgroup $G\left(\prod_{v \text { finite }} \mathcal{O}_{v}\right)$ of $G\left(\mathbb{A}_{f}\right)$.

A complex-valued function $f$ on $G(\mathbb{A})$ is smooth if it is continuous and, when viewed as a function of two arguments $(x, y)$ as in (7.2), it is smooth in $x$ for each fixed $y$ and is locally constant of compact support in $y$ for each fixed $x$. Let $\rho$ be a finite-dimensional representation of $K_{\infty}$, let $J \subset Z(\mathfrak{g})$ be an ideal of finite codimension, and let $K$ be an open subgroup of $K_{1}$. A smooth function $f$ on $G(\mathbb{A})$ is automorphic relative to $(\rho, J, K)$ if

(i) $f(\gamma g)=f(g)$ for all $\gamma \in G(F)$

(ii) $f(g k)=f(g)$ for all $k \in K$

(iii) the span of the right translates of $f$ by members of $K_{\infty}$ is finite-dimensional, and every irreducible constituent of this representation of $K_{\infty}$ is a constituent of $\rho$

(iv) the ideal $J$, acting in the $G_{\infty}$ variable of (7.2), has $J f=0$

(v) for each $y \in G\left(\mathbb{A}_{f}\right)$, the function $x \mapsto f(x y)$ on $G_{\infty}$ satisfies a certain slow-growth condition.

We shall not make (v) any more precise, but instead we refer to [Bo-Ja]; Theorem 7.3 below will clarify condition (v) in the principal case of interest. The set of automorphic functions relative to $(\rho, J, K)$ will be denoted $\mathcal{A}(\rho, J, K)$.

When $G=G L_{1}$, any Grossencharacter gives an example of an automorphic form relative to a suitable triple.

Theorem 7.1. For every $(\rho, J, K), \mathcal{A}(\rho, J, K)$ is finite-dimensional.

This theorem is fundamental. It is due to Harish-Chandra; see [HC1] and [HC2, p. 8], where it is proved in the setting of the right side of (6.3). The translation into the current setting (which is the left side of (6.3)) is in [Bo-Ja, p. 195].

Theorem 7.2. Let a smooth function $f$ on $G(\mathbb{A})$ satisfy (i) through (iv) above and also

$$
f(z x)=\chi(z) f(x) \quad \text { for all } z \in Z(\mathbb{A}) \text { and } x \in G(\mathbb{A})
$$


for some (unitary) character of $Z(F) \backslash Z(\mathbb{A})$, so that $|f|$ may be regarded as a function on $(Z(\mathbb{A}) G(F)) \backslash G(\mathbb{A})$. If $|f|$ is in $L^{p}((Z(\mathbb{A}) G(F)) \backslash G(\mathbb{A}))$ for some $p \geq 1$, then $f$ satisfies condition $(\mathrm{v})$ and hence is an automorphic form.

Reference. See [Bo-Ja, pp. 191 and 195]. The proof makes use of Theorem 6.2 and $[\mathrm{HC} 2]$.

A cusp form is an automorphic form $f$ such that (7.3) holds for some unitary character $\chi$ of $Z(F) \backslash Z(\mathbb{A})$ and such that

$$
\int_{N(F) \backslash N(\mathbb{A})} f(n g) d n=0
$$

for the unipotent radical $N$ of every proper parabolic subgroup of $G$ and for all $g \in G(\mathbb{A})$. For $G=G L_{1}$, the condition (7.4) is empty, and therefore all unitary Grossencharacters are cusp forms for $G L_{1}$. The classical analytic cusp forms relative to $S L_{2}(\mathbb{Z})$ yield cusp forms for $G=G L_{2}$ in the sense of (7.4), and so do Hecke's cusp forms relative to the subgroup $\Gamma_{0}(N)$ of $S L_{2}(\mathbb{Z})$. For general $G$, let ${ }^{0} \mathcal{A}(\rho, J, K)$ be the space of cusp forms relative to $(\rho, J, K)$.

Theorem 7.3. Let a smooth function $f$ on $G(\mathbb{A})$ satisfy (i) through (iv) above, as well as the cuspidal condition (7.4) and the condition (7.3) for some (unitary) character of $Z(F) \backslash Z(\mathbb{A})$. Then the following conditions are equivalent:

(i) $f$ satisfies (v) and hence is a cusp form

(ii) $f$ is bounded

(iii) $|f|$ is in $L^{2}((Z(\mathbb{A}) G(F)) \backslash G(\mathbb{A}))$.

Actually there is a sharper result: A smooth function $f$ satisfying (i) through (iv) of an automorphic form, as well as the slow-growth condition in (v) and the condition (7.3) for some unitary $\chi$, is automatically rapidly decreasing as soon as it satisfies the cuspidal condition (7.4). We already noted this fact for classical cusp forms relative to $S L_{2}(\mathbb{Z})$. The result for general $G$ requires precise definitions of "slow growth" and "rapidly decreasing," which we omit.

We want to define the notion of an automorphic representation of $G(\mathbb{A})$. Put $\mathcal{A}=\mathcal{A}(\rho, J, K)$. The idea is that an automorphic representation is any irreducible subquotient of $\mathcal{A}$, but the trouble is that $\mathcal{A}$ need not be mapped to itself under right translation by $G(\mathbb{A})$. Specifically, $K_{\infty}$ finiteness need not be preserved under right translation by $G_{\infty}$.

The idea is to make $\mathcal{A}$ into a module for an algebra $\mathcal{H}$ (the Hecke algebra) that reflects the action by $G\left(F_{v}\right)$ for each finite place $v$ and reflects the action by $U(\mathfrak{g})$ and $K_{\infty}$ at the infinite places. We summarize the construction, which is given in more detail in [Fl]. For each finite place $v$, let $\mathcal{H}_{v}$ be the algebra (under convolution) of all complex-valued locally constant functions of compact support on $G\left(F_{v}\right)$. Haar measure on $G\left(F_{v}\right)$ is to be normalized so that $G\left(\mathcal{O}_{v}\right)$ has measure 1 , and then the characteristic function $I_{v}$ of $G\left(\mathcal{O}_{v}\right)$ is an idempotent in $\mathcal{H}_{v}$. The algebra $\mathcal{H}_{v}$ contains a directed system of further idempotents, namely the normalized characteristic function of each open subgroup of $G\left(\mathcal{O}_{v}\right)$. An $\mathcal{H}_{v}$ module is approximately unital if, for each member of the module, all idempotents corresponding to sufficiently small open subgroups act as the identity. It is fairly easy to see that smooth representations of $G\left(F_{v}\right)$, i.e., those in which each vector is fixed by some compact open subgroup, correspond exactly to approximately unital 
$\mathcal{H}_{v}$ modules. Such a representation is called admissible if the set of vectors fixed by any compact open subgroup is finite-dimensional.

There is a natural way of forming a restricted tensor product of the algebras $\mathcal{H}_{v}$ with respect to the idempotents $I_{v}$. The resulting algebra $\mathcal{H}_{f}$ is the part of $\mathcal{H}$ corresponding to the finite places of $F$ and is generated by product functions that equal $I_{v}$ at almost every place. A tuple of local idempotents, one for each $\mathcal{H}_{v}$ with almost all of them being $I_{v}$, yields another idempotent in $\mathcal{H}_{f}$, and the idempotents obtained in this way are indexed by a directed set. A right $\mathcal{H}_{f}$ module is smooth if each member of the module is fixed by all idempotents corresponding to members of the directed set that are sufficiently large. The module is admissible if the set of vectors fixed by any of these idempotents is finite-dimensional.

Next we let $\mathcal{H}_{\infty}$ be the convolution algebra of all $K_{\infty}$ finite distributions on $G_{\infty}$ that are supported on $K_{\infty}$. This algebra is studied extensively in [Kna-Vo]. It contains a directed family of idempotents as follows. Let $d k$ denote normalized Haar measure on $K_{\infty}$. For each class of irreducible representations $\tau$ of $K_{\infty}$, let $\chi_{\tau}$ be the character and let $d_{\tau}$ be the degree. The directed family of idempotents is indexed by all finite subsets of $\tau$ 's, the idempotent corresponding to a given set being the sum of $d_{\tau} \chi_{\tau}$ for all $\tau$ in the set. A right $\mathcal{H}_{\infty}$ module is approximately unital if, for each member of the module, all sufficiently large idempotents act as the identity. It is shown in [Kna-Vo, pp. 75 and 90$]$ that $\left(\mathfrak{g}, K_{\infty}\right)$ modules correspond exactly to approximately unital $\mathcal{H}_{\infty}$ modules. Such a module is admissible if the set of vectors fixed by any of these idempotents is finite-dimensional, i.e., if each $K_{\infty}$ type has finite multiplicity.

We define $\mathcal{H}=\mathcal{H}_{\infty} \otimes \mathcal{H}_{f}$. Smoothness and admissibility of right $\mathcal{H}$ modules are defined using idempotents that are pure tensors from $\mathcal{H}_{\infty}$ and $\mathcal{H}_{f}$. Then $\mathcal{A}$ is a smooth right $\mathcal{H}$ module. An automorphic representation of $\mathcal{H}$ is any irreducible subquotient of $\mathcal{A}$. Similarly if we put ${ }^{0} \mathcal{A}={ }^{0} \mathcal{A}(\rho, J, K)$, then a cuspidal automorphic representation of $\mathcal{H}$ is any irreducible subquotient of ${ }^{0} \mathcal{A}$.

If $f$ is an automorphic form, then it is immediate from Theorem 7.1 that $f * \mathcal{H}$ is a smooth admissible $\mathcal{H}$ module. It follows that every automorphic representation of $\mathcal{H}$ is smooth and admissible. Such representations are commonly called automorphic representations of $G(\mathbb{A})$ even though not all of $G(\mathbb{A})$ really acts.

More generally a topologically irreducible $G(\mathbb{A})$ module is said to be automorphic if its underlying space of smooth vectors is an automorphic representation of $\mathcal{H}$. According to [Fl, Theorem 4], if $\chi$ is any (unitary) character of $Z(F) \backslash Z(\mathbb{A})$, then any $G(\mathbb{A})$ invariant irreducible closed subspace of

$$
\begin{aligned}
& L^{2}(G(F) \backslash G(\mathbb{A}))_{\chi} \\
& =\left\{f|| f \mid \in L^{2}((Z(\mathbb{A}) G(F)) \backslash G(\mathbb{A})) \text { and } f(z x)=\chi(z) f(x) \text { for } z \in Z(\mathbb{A}), x \in G(\mathbb{A})\right\}
\end{aligned}
$$

is automorphic in this sense.

Theorem 7.4. The subspace of cuspidal functions in $L^{2}(G(F) \backslash G(\mathbb{A}))_{\chi}$ decomposes discretely with finite multiplicities. Consequently whenever $f$ is a cusp form, $f * \mathcal{H}$ is a finite direct sum of cuspidal automorphic representations.

Reference. This theorem is due to Gelfand and Piatetski-Shapiro [Gelf-Pi]. See [Gelb1, p. 33] for a discussion when $G=G L_{2}$, and see [HC2, p. 9] for the general case. 
REMARK. It follows from the theorem that cuspidal automorphic representations are unitarizable. That is, they are the underlying smooth representations for irreducible unitary representations of $G(\mathbb{A})$.

Hecke's cusp forms discussed earlier in the section lead to cusp forms in the adelic sense, by Theorem 7.3. Those whose $L$ function has an Euler product expansion lead to adelic cusp forms that generate single (irreducible) cuspidal automorphic representations.

\section{Langlands Theory for $G L_{n}$}

In this section we describe what the Langlands theory proposes for $G L_{n}$. The theory for $G=G L_{n}$ may be regarded as a special case of the general theory, which will be discussed in the next section.

Fix a number field $F$. The theory wants to associate to each automorphic representation of $G L_{n}\left(\mathbb{A}_{F}\right)$ an $L$ function given initially as a product of elementary $L$ factors, one for each place of $F$. The method is arranged to be a direct generalization of the way in which an $L$ function is attached to a Grossencharacter in (5.2), the case of a unitary Grossencharacter being the special case $n=1$. At the same time it generalizes the way in which an $L$ function is attached to a classical cusp form, except that the starting point is the Euler product expansion and not the series expansion; this situation is an instance of the special case $n=2$ of the Langlands theory. For general $n$ and a given automorphic representation of $G L_{n}\left(\mathbb{A}_{F}\right)$, the Langlands theory gives a precise definition of the elementary $L$ factors at almost every place, and their product is convergent in a half plane. The definition at the remaining finite set of places hinges on a conjecture known as the Local Langlands Conjecture, which we shall discuss shortly.

By a theorem of Flath $[\mathrm{Fl}]$, any irreducible smooth admissible representation $\pi$ of $G L_{n}\left(\mathbb{A}_{F}\right)$ (or, more precisely, of the Hecke algebra) is a "restricted tensor product" $\pi=\bigotimes_{v} \pi_{v}$ of irreducible smooth admissible representations $\pi_{v}$ of the respective factors $G L_{n}\left(F_{v}\right)$ of $G L_{n}\left(\mathbb{A}_{F}\right)$. To define the $L$ function of $\pi$, it is therefore enough to describe the elementary $L$ factor of the representation $\pi_{v}$ of $G L_{n}\left(F_{v}\right)$.

Thus let $k$ be a local field of characteristic 0 , and let $\pi$ be an irreducible representation of $G L_{n}(k)$. Approximately, the theory proposes attaching to $\pi$ a continuous homomorphism $\varphi: W_{k} \rightarrow G L_{n}(\mathbb{C})$ with certain properties. Here $W_{k}$ is the Weil group of $k$. Then the $L$ factor for $\pi$ is defined to be a certain elementary function attached to $\varphi$ that generalizes any of the factors on the right side of (5.2).

The above description is correct if $k$ is archimedean, but the use of the Weil group $W_{k}$ is insufficient if $k$ is nonarchimedean. For reasons that we illustrate by example later in this section, the group $W_{k}$ in the nonarchimedean case is replaced by the Weil-Deligne group $W_{k}^{\prime}$, which we shall define now.

Let $\theta_{k}: k^{\times} \rightarrow W_{k}^{\mathrm{ab}}$ be the local reciprocity map of Corollary 2.5. Let $\|w\|$ denote the effect on $w \in W_{k}$ of the composition of passing from $W_{k} \rightarrow W_{k}^{\text {ab }}$, followed by passing to $k^{\times}$by $\theta_{k}^{-1}$, followed by passing to $\mathbb{R}_{+}^{\times}$by $|\cdot|_{k}$. The Weil-Deligne group $W_{k}^{\prime}$ is the semidirect product of $W_{k}$ and $\mathbb{C}$, where $W_{k}$ acts on $\mathbb{C}$ by $w x w^{-1}=\|w\| x$. Thus the multiplication rule on $\mathbb{C} \times W_{k}$ is $\left(a_{1}, w_{1}\right)\left(a_{2}, w_{2}\right)=\left(a_{1}+\left\|w_{1}\right\| a_{2}, w_{1} w_{2}\right)$.

In the nonarchimedean case, a continuous homomorphism $\varphi: W_{k}^{\prime} \rightarrow G L_{n}(\mathbb{C})$ is called an admissible homomorphism if $\varphi$ is holomorphic in the $\mathbb{C}$ variable, if $\varphi(\mathbb{C})$ consists of unipotent matrices, and if $\varphi\left(W_{k}\right)$ consists of semisimple matrices. Such a homomorphism amounts to specifying a pair $(\rho, X)$, where 
$\rho: W_{k} \rightarrow G L(n, \mathbb{C})$ is a continuous homomorphism such that $\varphi\left(W_{k}\right)$ consists of semisimple matrices and $X$ is a nilpotent endomorphism of $\mathbb{C}^{n}$ such that $\rho(w) X \rho(w)^{-1}=\|w\| X$ for all $w \in W_{k}$. Let Fr be an element of $W_{k}$ such that $\|\operatorname{Fr}\|=q^{-1}$, where $q$ is the order of the residue field; the element $\mathrm{Fr}$ is uniquely determined modulo the inertia group $I_{k}$ of (1.4). If $V_{X}^{I}$ is the subspace of ker $X$ fixed by $\rho\left(I_{k}\right)$, then the elementary $L$ factor associated to $\varphi$ is ${ }^{4}$

$$
L(s, \varphi)=\operatorname{det}\left(1-\left(\left.\rho(\mathrm{Fr})\right|_{V_{X}^{I}}\right) q^{-s}\right)^{-1} .
$$

Parenthetically let us mention a substitute for the Weil-Deligne group that one encounters in the literature. With the right definition the admissible homomorphisms into $G L_{n}(\mathbb{C})$ for the substitute group will correspond to the admissible homomorphisms of $W_{k}^{\prime}$, and ultimately no semidirect products will be involved in the definition of the substitute group. For $w \in W_{k}$, let $h_{w}$ be the matrix $\left(\begin{array}{cc}\|w\|^{1 / 2} & 0 \\ 0 & \|w\|^{-1}\end{array}\right)$ in $S L_{2}(\mathbb{C})$, and identify $z \in \mathbb{C}$ with $\left(\begin{array}{ll}1 & z \\ 0 & 1\end{array}\right)$. The above action of $W_{k}$ on $\mathbb{C}$ by automorphisms translates into conjugation of $\left(\begin{array}{ll}1 & z \\ 0 & 1\end{array}\right)$ by $h_{w}$. Since conjugation by $h_{w}$ extends to an automorphism of all of $S L_{2}(\mathbb{C})$, we can identify the action of $W_{k}$ on $\mathbb{C}$ with a subaction of the action of $W_{k}$ on $S L_{2}(\mathbb{C})$ by automorphisms. The semidirect product of $S L_{2}(\mathbb{C})$ by $W_{k}$ with respect to this action then consists of pairs $(x, w)$ with $x \in S L_{2}(\mathbb{C})$ and $w \in W_{k}$, where $\left(x_{1}, w_{1}\right)\left(x_{2}, w_{2}\right)=\left(x_{1} h_{w_{1}} x_{2} h_{w_{1}}^{-1}, w_{1} w_{2}\right)$. This group is isomorphic to the direct product $S L_{2}(\mathbb{C}) \times W_{k}$ by $(x, w) \mapsto\left(x h_{w}, w\right)$, and $S L_{2}(\mathbb{C}) \times W_{k}$ is then used as the substitute for the Weil-Deligne group. We shall not use this substitute, however.

In the archimedean case, an admissible homomorphism $\varphi: W_{k} \rightarrow G L_{n}(\mathbb{C})$ is just a continuous homomorphism such that $\varphi\left(W_{k}\right)$ consists of semisimple matrices. The elementary $L$ factor associated to such a $\varphi$ is a nowhere-zero meromorphic function involving $\Gamma$ functions and may be found in [Kna2, p. 404].

Two admissible homomorphisms $\varphi_{1}$ and $\varphi_{2}$ are said to be equivalent if they are conjugate via $G L_{n}(\mathbb{C})$, i.e., if there exists $g \in G L_{n}(\mathbb{C})$ with $g \varphi_{1}(x) g^{-1}=\varphi_{2}(x)$ for all $x$ in the Weil-Deligne group or Weil group, as appropriate. The set of equivalence classes of admissible homomorphisms is denoted $\Phi\left(G L_{n}(k)\right)$.

For each local field $k$ of characteristic 0 , let $\Pi\left(G L_{n}(k)\right)$ be the set of equivalence classes of (smooth) irreducible admissible representations of $G L_{n}(k)$. If $k$ is nonarchimedean, "equivalence" here means equivalence as representations of the group (or of the Hecke algebra). If $k$ is archimedean, "equivalence" means infinitesimal equivalence (or equivalence as representations of the Hecke algebra). Leaving aside some further definitions for the moment, we can state the Local Langlands Conjecture as follows.

Local Langlands Conjecture. $\Pi\left(G L_{n}(k)\right)$ is indexed by $\Phi\left(G L_{n}(k)\right)$ in a natural way that is compatible with twisting by Grossencharacters and respects $L$ factors and $\varepsilon$ factors.

For $\varphi \in \Phi\left(G L_{n}(k)\right)$ and $\pi \in \Pi\left(G L_{n}(k)\right)$, the "twists" $\varphi \otimes \alpha$ and $\pi \otimes \alpha$ by a Grossencharacter $\alpha$ are defined toward the end of this section. The requirement about twists is that if $\varphi$ corresponds to $\pi$, then $\varphi \otimes \alpha$ corresponds to $\pi \otimes \alpha$ for every $\alpha$.

\footnotetext{
${ }^{4}$ See the footnote for (5.1a).
} 
Let us discuss the requirement on $L$ factors and $\varepsilon$ factors. We can define the Langlands elementary $L$ factor of an irreducible $\pi$ to be the elementary $L$ factor of the corresponding $\varphi$ :

$$
L(s, \pi)=L(s, \varphi) \quad \text { if } \varphi \leftrightarrow \pi,
$$

with $L(s, \varphi)$ as in (8.1). In Jacquet's lecture [Ja2] may be found a completely different construction of elementary $L$ factors attached to irreducible admissible representations (due to Godement and Jacquet [Go-Ja]), and this construction does not depend on any conjectures. We require that the two kinds of $L$ factors match. Properties of the Godement-Jacquet $L$ functions will be discussed below after Corollary 8.6.

We mentioned $\varepsilon$ functions attached to Galois representations in connection with Theorem 5.3. These are products of local $\varepsilon$ factors that we have not defined, are 1 at almost every place, and generalize the Gauss sums that occur in the functional equations of Dirichlet $L$ functions [Kna1, p. 216]. Local $\varepsilon$ factors depend upon additional data, but let us suppress this point. In a fashion similar to that for Galois representations, a local $\varepsilon$ factor may be associated to each $\varphi \in \Phi\left(G L_{n}(k)\right)$. The correspondence of the Local Langlands Conjecture then allows one to define a Langlands $\varepsilon$ factor for $\pi \in \Pi\left(G L_{n}(k)\right)$ by

$$
\varepsilon(s, \pi)=\varepsilon(s, \varphi) \quad \text { if } \varphi \leftrightarrow \pi .
$$

Meanwhile the Godement-Jacquet construction also defines an $\varepsilon$ factor for each $\pi$ in $\Pi\left(G L_{n}(k)\right)$. We require that the two constructions match.

The paper [Kud, p. 380] gives a full list of the requirements that the conjectural local Langlands correspondence is supposed to satisfy.

The Local Langlands Conjecture is a theorem when $n=1$. In this case the result amounts to a restatement of Corollary 2.5. For general $n$ and for $k$ archimedean, the conjecture is a theorem of Langlands; see [Kna2] for an exposition. Much is known in the nonarchimedean case. See [Kud] for an exposition. The conjecture is known for $n=2$ ([Kut] and [Tu]), for $n=3$ ([Hen1]), and for all relatively prime $n$ and $p$ [Moy]. For more recent work, see [Ha]. Henniart [Hen2] has shown that there is at most one candidate for the local Langlands correspondence respecting $\varepsilon$ factors for "pairs" in the sense of [Ja-P-S]. See [Kud, §4.2] for more detail.

A part of the correspondence is easy to understand. For definiteness, let $k$ be nonarchimedean. Let $\rho$ be a continuous homomorphism of $W_{k}$ into the diagonal subgroup of $G L_{n}(\mathbb{C})$. Composing with projection to each diagonal entry, we see that $\rho$ is completely determined by $n$ quasicharacters $\chi_{1}, \ldots, \chi_{n}$ of $W_{k}^{\text {ab }} \cong k^{\times}$. Let us write $\rho=\rho_{\left(\chi_{1}, \ldots, \chi_{n}\right)}$. If we put $X=0$, then

$$
\varphi=\varphi_{\left(\chi_{1}, \ldots, \chi_{n}\right)}=\left(\rho_{\left(\chi_{1}, \ldots, \chi_{n}\right)}, 0\right)
$$

is an admissible homomorphism. Let $T$ be the diagonal subgroup of $G L_{n}(k)$, and let $N$ be the upper-triangular subgroup with 1's on the diagonal. The data $\left(\chi_{1}, \ldots, \chi_{n}\right)$ give us a quasicharacter of $T$, and it is natural to associate to $\varphi_{\left(\chi_{1}, \ldots, \chi_{n}\right)}$ the induced representation

$$
\operatorname{ind}_{T N}^{G L_{n}(k)}\left(\left(\chi_{1}, \ldots, \chi_{n}\right) \otimes 1\right)
$$

given by normalized induction (a member of the nonunitary principal series). This representation may be taken to be the $\pi$ that corresponds to $\varphi$ when $\pi$ is 
irreducible, and it is known that irreducibility occurs if and only if there is no pair of indices $i$ and $j$ such that $\chi_{i} \chi_{j}^{-1}$ equals $|\cdot|_{k}$.

When (8.3) is reducible, the $\pi$ that is associated to $\varphi_{\left(\chi_{1}, \ldots, \chi_{n}\right)}$ is a certain irreducible subquotient of (8.3) known as the Langlands subquotient. To describe the Langlands subquotient, we first remark that the set of irreducible subquotients of (8.3) is unchanged (apart from equivalence) when $\chi_{1}, \ldots, \chi_{n}$ are permuted. In order to have the indexing $\Phi \leftrightarrow \Pi$ depend only on equivalence classes, we require that the Langlands subquotient not be affected by permutation. Next, we can introduce complex numbers $s_{1}, \ldots, s_{n}$ such that $\left|\chi_{i}\right|=|\cdot|{ }_{k}^{s_{i}}$. The numbers $s_{i}$ are not unique, but their real parts are unique. If the real parts of $s_{1}, \ldots, s_{n}$ are nonincreasing, then the Langlands subquotient is the (unique) irreducible quotient of (8.3). The result is that the Langlands subquotient is determined in every case. See [Moe2] in this volume for further discussion.

A special case of the nonunitary principal series of particular interest is the unramified principal series, those members of the nonunitary principal series having a nonzero fixed vector under $G L_{n}\left(\mathcal{O}_{k}\right)$. By Frobenius reciprocity these are just the representations (8.3) for which each $\chi_{i}(x)$ depends only on the module of $x$. Thus each $\chi_{i}$ is of the form $|\cdot|^{s_{i}}$ for some complex $s_{i}$. The Langlands subquotient of an unramified principal series is the irreducible subquotient containing a nonzero vector fixed by $G L_{n}\left(\mathcal{O}_{k}\right)$. All members of $\Pi\left(G L_{n}(k)\right)$ having a nonzero vector fixed by $G L_{n}\left(\mathcal{O}_{k}\right)$ are of this form, up to equivalence. Up to equivalence, they are parametrized by orbits of the symmetric group on tuples $\left(s_{1}, \ldots, s_{n}\right)$ of complex numbers modulo $2 \pi i(\log q)^{-1} \mathbb{Z}$. In view of $(8.2)$, the elementary $L$ factor of Langlands is well defined in the case of a member of $\Pi\left(G L_{n}(k)\right)$ having a nonzero vector fixed by $G L_{n}\left(\mathcal{O}_{k}\right)$. Such a member of $\Pi\left(G L_{n}(k)\right)$ is said to be unramified.

Let us now discuss the need for the Weil-Deligne group $W_{k}^{\prime}$ rather than just the Weil group $W_{k}$. Suppose that $n=2$. The irreducible admissible representations of $G L_{2}(k)$ are then of three kinds - the supercuspidal representations (those whose matrix coefficients are compactly supported modulo the center), the Langlands subquotients of the nonunitary principal series, and the special representations. In (8.3), reducibility occurs for $G L_{2}(k)$ exactly when $\chi_{1} \chi_{2}^{-1}=|\cdot|_{k}^{ \pm 1}$. In this case, there are two irreducible subquotients, the Langlands subquotient and one other. These "other" representations are the special representations. Their matrix coefficients are square integrable modulo the center, but these representations are not supercuspidal.

For $n=2$, the Godement-Jacquet $L$ factors of supercuspidal representations are 1 , of special representations involve one factor with $q^{-s}$ in it, and of Langlands subquotients of nonunitary principal series involve two factors with $q^{-s}$ in them. For the Local Langlands Conjecture to be valid, it is necessary to arrange for some other two-dimensional representations (of $W_{k}$ or a substitute) than the irreducible ones (which give 1 as $L$ factor) and the direct sums of one-dimensional ones (which give $L$ factors that are the product of two expressions involving $q^{-s}$ ). Englargement to the Weil-Deligne group allows the existence of indecomposable yet reducible twodimensional representations of $W_{k}^{\prime}$. For one of these representations, the space is $\mathbb{C}^{2}$ with a basis $e_{1}, e_{2}$ and with action

$$
\rho(w) e_{i}=\|w\|^{i-1} e_{i}, \quad X e_{1}=e_{2}, \quad X e_{2}=0 .
$$

The most general indecomposable yet reducible two-dimensional representation of $W_{k}^{\prime}$, up to equivalence, is the tensor product of this one with a one-dimensional 
representation. The equivalence classes of indecomposable two-dimensional representations are used to parametrize the special representations up to equivalence.

Use of $W_{k}^{\prime}$ to settle $G L_{2}(k)$ looks somewhat artificial, but $W_{k}^{\prime}$ looks more reasonable when one considers what is known about $G L_{n}$. For $G L_{n}(k)$ with $k$ nonarchimidean, the irreducible admissible representations have been classified. The starting point is the supercuspidal representations, which have been classified by Bushnell-Kutzko and by Corwin. Bernstein and Zelevinski showed how to classify the irreducible admissible representations in terms of the supercuspidal representations. See [Kud] and [Moe2] for more detail and for references. The nature of the Bernstein-Zelevinski part of the classification implies that if the supercuspidal representations of $G L_{n}(k)$ correspond to the $n$-dimensional irreducible admissible homomorphisms $W_{k} \rightarrow G L_{n}(\mathbb{C})$ of the Weil group $W_{k}$ for all $n$, then $\Pi\left(G L_{n}(k)\right)$ is parametrized by the $n$-dimensional admissible homomorphisms $W_{k}^{\prime} \rightarrow G L_{n}(\mathbb{C})$ of the Weil-Deligne group $W_{k}^{\prime}$. (See [Ja1, (3.7)] and [Kud, p. 381].)

Now we return to the number field $F$. Let $\pi=\bigotimes_{v} \pi_{v}$ be an irreducible admissible representation of $G L_{n}\left(\mathbb{A}_{F}\right)$. Then we can define the Langlands $L$ functions by

$$
\begin{aligned}
& L(s, \pi)=\prod_{\text {finite } v} L\left(s, \pi_{v}\right) \\
& \Lambda(s, \pi)=\prod_{\text {all } v} L\left(s, \pi_{v}\right),
\end{aligned}
$$

where the factors are given by (8.2) and ultimately (8.1). The first question is one of convergence. Built into the factorization of $\pi$ as $\bigotimes_{v} \pi_{v}$ is the following addendum, which simplifies questions of convergence greatly.

Theorem 8.1. If $\pi=\bigotimes_{v} \pi_{v}$ is an irreducible admissible representation of $G L_{n}\left(\mathbb{A}_{F}\right)$, then almost every $\pi_{v}$ is unramified, i.e., has a nonzero fixed vector under $G L_{n}\left(\mathcal{O}_{v}\right)$ and is therefore the Langlands subquotient of an unramified principal series.

REFERENCE. [Fl, p. 181].

Corollary 8.2. If $\pi$ is a unitarizable irreducible admissible representation of $G L_{n}\left(\mathbb{A}_{F}\right)$, then $L(s, \pi)$ converges absolutely for $\operatorname{Re} s$ sufficiently large.

Reference. This is essentially due to Langlands. See [Bo4, p. 50].

In fact, Theorem 8.1 shows that it is enough to consider the factors of $\pi$ that are unramified principal series. Flath's results show that we may take each factor to be unitarizable, and then all that is needed is an estimate on the $L$ factor for a unitarizable unramified principal series.

Corollary 8.3. If $\pi$ is a cuspidal automorphic representation of $G L_{n}\left(\mathbb{A}_{F}\right)$, then $L(s, \pi)$ converges absolutely for $\operatorname{Re} s$ sufficiently large.

REFEREnCE. This kind of result is due to Langlands [Lgl2] and predates [Fl]. The proof is immediate from the above results: The cuspidal representation is unitarizable by Theorem 7.4, and convergence follows from Corollary 8.2.

With considerably more effort, Langlands has addressed convergence of $L(s, \pi)$ for general automorphic $\pi$. We need another corollary of Theorem 8.1. 
Corollary 8.4. Let $P=M N$ be the usual Levi decomposition of a standard parabolic subgroup of $G L_{n}$. Let $\sigma$ be a unitarizable irreducible admissible representation of $M\left(\mathbb{A}_{F}\right)$, and let $\pi$ be an irreducible subquotient of $\operatorname{ind}_{M\left(\mathbb{A}_{F}\right) N\left(\mathbb{A}_{F}\right)}^{G L_{n}\left(\mathbb{A}_{F}\right)}(\sigma \otimes 1)$. Then $L(s, \pi)$ converges absolutely for $\operatorname{Re} s$ sufficiently large.

Reference. [Bo4, p. 52].

Theorem 8.5. An irreducible admissible representation $\pi$ of $G L_{n}\left(\mathbb{A}_{F}\right)$ is automorphic if and only if $\pi$ is equivalent with an irreducible subquotient of

$$
\operatorname{ind}_{M\left(\mathbb{A}_{F}\right) N\left(\mathbb{A}_{F}\right)}^{G L_{n}\left(\mathbb{A}_{F}\right)}(\sigma \otimes 1)
$$

for some cuspidal automorphic representation $\sigma$ of $M\left(\mathbb{A}_{F}\right)$.

REFERENCE. [Lgl4, p. 204].

Corollary 8.6. If $\pi$ is an automorphic representation of $G L_{n}\left(\mathbb{A}_{F}\right)$, then $L(s, \pi)$ converges absolutely for $\operatorname{Re} s$ sufficiently large.

Reference. This result of Langlands combines the above ideas. See [Bo4, p. 52]. By Theorem 8.5, $\pi$ is a constituent of a representation induced from a cuspidal representation, and the cuspidal representation is unitarizable by Theorem 7.4. Then convergence follows from Corollary 8.4.

The next question concerns the analytic properties of automorphic representations. We shall be content with the results in the cuspidal case. For the general automorphic case, see [Ja1, p. 83]. As we mentioned above, Godement and Jacquet [Go-Ja] have constructed $L$ and $\Lambda$ functions in a way that is completely different from using the Local Langlands Conjecture. Their theory is summarized in this volume in [Ja2], and conjecturally their functions, which we denote $L^{\mathrm{GJ}}(s, \pi)$ and $\Lambda^{\mathrm{GJ}}(s, \pi)$, coincide with those in (8.4). What is known is that the GodementJacquet $L$ functions agree with the Langlands $L$ functions at every place where $\pi_{v}$ is unramified. (This condition holds for almost every place by Theorem 8.1.) The Godement-Jacquet $L$ functions have good global analytic properties, as follows.

Theorem 8.7. If $\pi$ is a cuspidal representation of $G L_{n}\left(\mathbb{A}_{F}\right)$, the GodementJacquet function $\Lambda^{\mathrm{GJ}}(s, \pi)$ extends to be meromorphic in $\mathbb{C}$ with singularities given at most by simple poles at $s=0$ and $s=1$. Moreover, $\Lambda^{\mathrm{GJ}}(s, \pi)$ satisfies a functional equation of the form

$$
\Lambda^{\mathrm{GJ}}(s, \pi)=\varepsilon^{\mathrm{GJ}}(s, \pi) \Lambda^{\mathrm{GJ}}\left(1-s, \pi^{\vee}\right),
$$

where $\pi^{\vee}$ is the contragredient and $\varepsilon^{\mathrm{GJ}}(s, \pi)$ is a multiple of $N^{-s}$ for some integer $N$. The function $\Lambda^{\mathrm{GJ}}(s, \pi)$ is actually entire unless $\pi$ is a unitary character of $G L_{1}\left(\mathbb{A}_{F}\right)$ trivial on the elements of module 1.

When $n=1$, the $L$ functions in Theorem 8.7 (with the elementary factors from the infinite places dropped) are the ones attached to unitary Grossencharacters by Hecke and Tate, and Theorem 8.7 generalizes the work in Tate's thesis [Ta1]. When $n=2$, these $L$ functions (after a change of parameter) generalize the $L$ functions attached by Hecke to cusp forms relative to $S L_{2}(\mathbb{Z})$; Hecke proved that his $L$ functions are entire and satisfy a functional equation. 
An important aspect of the Langlands theory for $G L_{n}$ is a reciprocity conjecture that implies the Artin Conjecture (end of $\S 5$ ). We state this conjecture of Langlands in two forms - the first version involving almost all places and either Langlands $L$ functions or Godement-Jacquet $L$ functions, the second version involving all places and Godement-Jacquet $L$ functions. For the second version we need to complete the Artin $L$ function $L(s, \sigma)$ to a function $\Lambda(s, \sigma)$ by adjoining elementary $L$ factors for the infinite places; these factors are listed explicitly in [Kna2, (3.6) and (4.6)].

Langlands Reciprocity Conjecture, first version. For any irreducible representation $\sigma$ of $\operatorname{Gal}(\bar{F} / F)$ in $G L_{n}(\mathbb{C})$, there exists a cuspidal automorphic representation $\pi$ of $G L_{n}\left(\mathbb{A}_{F}\right)$ such that the Artin $L$ function of $\sigma$ agrees with the Langlands $L$ function of $\pi$ at almost every place where $\pi$ is unramified.

Langlands Reciprocity Conjecture, second version. For any irreducible representation $\sigma$ of $\operatorname{Gal}(\bar{F} / F)$ in $G L_{n}(\mathbb{C})$, there exists a cuspidal automorphic representation $\pi$ of $G L_{n}\left(\mathbb{A}_{F}\right)$ such that the completed Artin $L$ function $\Lambda(s, \sigma)$ of $\sigma$ is identical with the Godement-Jacquet $L$ function $\Lambda^{\mathrm{GJ}}(s, \pi)$ of $\pi$. In more detail, $L_{v}(s, \sigma)=L_{v}^{\mathrm{GJ}}(s, \pi)$ for every finite place $v$, and $L_{\infty}(s, \sigma)=L_{\infty}^{\mathrm{GJ}}(s, \pi)$ if $L_{\infty}$ and $L_{\infty}^{\mathrm{GJ}}$ denote the products of the factors for the infinite places.

If $\pi=\bigotimes_{v} \pi_{v}$, then we have noted that the Langlands $L$ factor for $\pi_{v}$ agrees with the Godement-Jacquet $L$ factor for $\pi_{v}$ when $\pi_{v}$ is unramified and that $\pi_{v}$ is unramified at almost every place. Therefore the second version of the conjecture implies the first. The converse is addressed by the following.

Theorem 8.8. The first version of the Langlands Reciprocity Conjecture for $\sigma$ implies the second for $\sigma$. Consequently either version of the Langlands Reciprocity Conjecture for $\sigma$ implies the Artin Conjecture (end of $\S 5$ ) for $\sigma$.

We postpone the discussion of Theorem 8.8 to the end of this section except to note that the second sentence of the theorem follows by combining Theorem 8.7 and the second version of the conjecture.

Let us discuss the conjecture itself. The basic case for which this conjecture is known is Theorem 5.1, which handles $n=1$. That theorem says that abelian Artin $L$ functions are Hecke $L$ functions of Grossencharacters. On the one hand, this result is essentially equivalent with Artin reciprocity. On the other hand, it establishes that the pattern of $\varphi_{\wp}\left(\pi_{\wp}\right)$, which governs the factorization of certain polynomials modulo $\wp$, is given in terms of arithmetic progressions in the abelian case. The general conjecture may therefore be regarded as a statement about reciprocity on the one hand and a statement about the pattern of factorization of polynomials modulo $\wp$ on the other hand.

It is natural to expect that the Langlands Reciprocity Conjecture holds for $\sigma=$ ind $\rho$ whenever it holds for $\rho$ and ind $\rho$ is irreducible. But it is an open problem to prove such a statement, even for $\rho$ one-dimensional. In the special case that $\rho$ is one-dimensional and the induced representation is two-dimensional, Jacquet and Langlands [Ja-Lgl] did manage to prove the statement, and it follows that the Langlands Reciprocity Conjecture holds when $n=2$ for $\sigma$ of "dihedral type" in the sense of $[$ Ro2, $\S 16]$.

In fundamental work [Lg15], Langlands handled some additional cases when $n=$ 2, and later Tunnell $[\mathrm{Tu}]$ was able to deduce an improved result using the methods of Langlands. These results of Langlands and Tunnell are the subject of the lectures [Ro2] by Rogawski. The statement is as follows. 
Theorem 8.9. If $\sigma$ is a two-dimensional complex representation of $\operatorname{Gal}(\bar{F} / F)$ with solvable image, then the Langlands Reciprocity Conjecture holds for $\sigma$.

For further work with $n=2$, see [Buhl] and [Fre].

Any initial attempt at proving the Langlands Reciprocity Conjecture raises the following question: If we have irreducible admissible representations $\pi_{v}$ for each place $v$ of $F$ and if $\pi=\bigotimes_{v} \pi_{v}$ is well defined, how do we tell whether $\pi$ is automorphic? In other words, how is the global behavior relative to $G L_{n}(F)$ reflected in the system of representations $\pi_{v}$ ? Theorem 8.1 gives a necessary condition; almost all $\pi_{v}$ must be Langlands subquotients of unramified principal series. What else can be said?

We are especially interested in the cuspidal case. If we are given an irreducible admissible $\pi=\bigotimes_{v} \pi_{v}$, the Multiplicity One Theorem below says that there is at most one way that $\pi$ can occur as a cuspidal representation. Recall from Theorem 7.4 that the cuspidal part of $L^{2}\left(G L_{n}(F) \backslash G L_{n}\left(\mathbb{A}_{F}\right)\right)_{\chi}$ decomposes discretely with finite multiplicities.

Theorem 8.10. If $\pi$ is an irreducible admissible representation of $G L_{n}\left(\mathbb{A}_{F}\right)$ occurring in the cuspidal part of $L^{2}\left(G L_{n}(F) \backslash G L_{n}\left(\mathbb{A}_{F}\right)\right)_{\chi}$ for some unitary character $\chi$, then $\pi$ occurs with multiplicity one.

REFERENCE. [Sha].

For two Grossencharacters $\chi=\bigotimes_{v} \chi_{v}$ and $\chi^{\prime}=\bigotimes_{v} \chi_{v}^{\prime}$, an equality $\chi_{v}=\chi_{v}^{\prime}$ for almost all $v$ implies $\chi=\chi^{\prime}$. In fact, we may assume that $\chi_{v}=1$ for almost all $v$. Applying the Weak Approximation Theorem (Theorem 3.7) and using that $\chi$ is 1 on $F^{\times}$and is continuous, we see that $\chi$ equals 1 everywhere. The following Strong Multiplicity One Theorem generalizes this result from $G L_{1}$ to $G L_{n}$.

Theorem 8.11. Let $\pi=\bigotimes_{v} \pi_{v}$ and $\pi^{\prime}=\bigotimes_{v} \pi_{v}^{\prime}$ be irreducible admissible representations of $G L_{n}\left(\mathbb{A}_{F}\right)$ occurring in the cuspidal part of $L^{2}\left(G L_{n}(F) \backslash G L_{n}\left(\mathbb{A}_{F}\right)\right)_{\chi}$ for some unitary character $\chi$. If $\pi_{v}$ is equivalent with $\pi_{v}^{\prime}$ for almost all $v$, then $\pi=\pi^{\prime}$.

Reference. [Ja-Sha, p. 553].

We do not conclude merely that $\pi$ and $\pi^{\prime}$ are equivalent. Theorem 8.10 allows us to deduce equality from equivalence. It follows from Theorem 8.11 that the cuspidal automorphic representation $\pi$ in the Langlands Reciprocity Conjecture is unique if it exists.

Thus cuspidal representations are rather rigid. But how do we tell when we have one? When $F=\mathbb{Q}$, we can phrase the question in a related way. If $L(s)$ is a Dirichlet series obtained by expanding out a product of elementary factors as in (8.1), when is $L(s)$ the $L$ function of a cuspidal automorphic representation? Weil [We2], generalizing work of Hecke, answered the question about when a Dirichlet series $\sum_{n=1}^{\infty} a_{n} / n^{s}$ comes from a cusp form $\sum_{n=1}^{\infty} a_{n} e^{2 \pi i n \tau}$ for some $\Gamma_{0}(N)$, and JacquetLanglands [Ja-Lgl] answered the corresponding question about general cuspidal representations of $G L_{2}$. Weil's result, known as the Weil Converse Theorem, says that if every twist $\sum_{n=1}^{\infty} a_{n} \chi(n) / n^{s}$ by a primitive Dirichlet character $\chi$ modulo $r$ with $r$ prime to $N$ extends to an entire function bounded in vertical strips and satisfying a suitable functional equation, then $\sum_{n=1}^{\infty} a_{n} e^{2 \pi i n \tau}$ is a cusp form for $\Gamma_{0}(N)$. 
The Jacquet-Langlands result [Ja-Lgl, p. 397] below uses the Weil group $W_{F}$ of the number field $F$, a notion treated in [Ta3] whose precise definition we do not need. We list the properties of $W_{F}$ that we shall use:

1) There is a natural continuous homomorphism $W_{F} \rightarrow \operatorname{Gal}(\bar{F} / F)$, and it of course induces a homomorphism $W_{F}^{\mathrm{ab}} \rightarrow \operatorname{Gal}(\bar{F} / F)^{\mathrm{ab}}$.

2) The Artin map $F^{\times} \backslash \mathbb{A}_{F}^{\times} \rightarrow \operatorname{Gal}(\bar{F} / F)^{\text {ab }}$ factors as

$$
F^{\times} \backslash \mathbb{A}_{F}^{\times} \stackrel{\sim}{\longrightarrow} W_{F}^{\mathrm{ab}} \longrightarrow \operatorname{Gal}(\bar{F} / F)^{\mathrm{ab}} .
$$

3) There is a canonical continuous homomorphism $j_{v}: W_{F_{v}} \rightarrow W_{F}$ such that the diagram

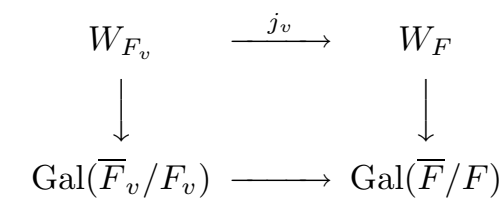

commutes.

4) The isomorphisms $F^{\times} \backslash \mathbb{A}_{F}^{\times} \stackrel{\sim}{\rightarrow} W_{F}^{\text {ab }}$ of $(2)$ and $F_{v}^{\times} \stackrel{\sim}{\rightarrow} W_{F_{v}}^{\text {ab }}$ of Corollary 2.5 are such that the diagram

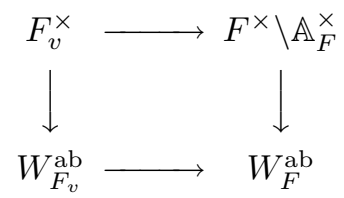

commutes. (This strengthens Theorem 4.5.)

The Jacquet-Langlands Converse Theorem also uses Weil's generalization of Artin $L$ functions in which a representation $\sigma$ of the Galois group $\operatorname{Gal}(\bar{F} / F)$ is replaced by an admissible homomorphism of the Weil group $W_{F}$ into some $G L_{n}(\mathbb{C})$. The local definition is in (8.1), and (3) says that it is consistent with Artin's definition. Further properties of the Weil $L$ functions $L(s, \sigma)$ and $\Lambda(s, \sigma)$ are given in [Ja-Lgl, pp. 393-394]. Like Artin $L$ functions, the Weil $L$ functions are known to have meromorphic continuations and to satisfy a functional equation (5.4). Thus a hypothesis about a functional equation need not be included in the theorem.

To state the theorem we need to explain a notion of twisting in this context. Let $\sigma$ be an $n$-dimensional representation of $\operatorname{Gal}(\bar{F} / F)$ or, more generally as a consequence of (1) above, of the Weil group $W_{F}$ of the number field $F$. Let $\alpha$ be a Grossencharacter.

The twist $\sigma \otimes \alpha$ will be an admissible $n$-dimensional representation of $W_{F}$. To define $\sigma \otimes \alpha$, all we have to do is interpret $\alpha$ as a one-dimensional representation of $W_{F}$. This we can do since $\alpha$ is a one-dimensional representation of $F^{\times} \backslash \mathbb{A}_{F}^{\times}$and this group is isomorphic with $W_{F}^{\text {ab }}$ by (2). Thus $\sigma \otimes \alpha$ is meaningful, and so are the Weil $L$ function $L(s, \sigma \otimes \alpha)$ and its completed version $\Lambda(s, \sigma \otimes \alpha)$. The decomposition $\alpha=\bigotimes \alpha_{v}$ is unambiguous in the two interpretations as a consequence of (4).

Theorem 8.12 (Jacquet-Langlands Converse Theorem). Suppose that $\sigma$ is a two-dimensional representation of $W_{F}$, and suppose that $\pi\left(\sigma_{v}\right)$ is the irreducible admissible representation of $G L_{2}\left(F_{v}\right)$ corresponding to $\sigma \circ j_{v}$. If, for every unitary Grossencharacter $\alpha$, the Weil L functions $\Lambda(s, \sigma \otimes \alpha)$ and $\Lambda\left(s, \sigma^{\vee} \otimes \omega^{-1}\right)$ are 
entire functions bounded in vertical strips, then $\bigotimes_{v} \pi\left(\sigma_{v}\right)$ is a cuspidal automorphic representation of $G L_{2}\left(\mathbb{A}_{F}\right)$.

A corresponding theorem is known for $G L_{3}$. See [Pi] and [Co-Pi] for results for $G L_{n}$ with $n \geq 4$, where additional hypotheses are needed. Converse theorems play a role in the work of Langlands on the Artin Conjecture; see Rogawski's lectures [Ro2]).

Let us return to Theorem 8.8. A proof for $n=2$ appears in [Lgl5, pp. 23-24]. See also [De-S]. The result for general $n$ appears to be a folk theorem, with no proof appearing in the literature. We are grateful to Dinakar Ramakrishnan for supplying us with the proof that follows and for giving permission that it be reproduced here.

Let $\sigma$ be an $n$-dimensional representation of $\operatorname{Gal}(\bar{F} / F)$, and let $\pi$ be a cuspidal automorphic representation of $G L_{n}\left(\mathbb{A}_{F}\right)$, with $\sigma$ and $\pi$ as in the first version of the Langlands Reciprocity Conjecture. We write $\sigma_{v}$ for the restriction of $\sigma$ to $\operatorname{Gal}\left(\bar{F}_{v} / F_{v}\right)$. The same representation $\pi$ of $G L_{n}\left(\mathbb{A}_{F}\right)$ will occur in the two versions of the conjecture, as it must by Theorem 8.11, and we decompose $\pi$ as $\pi=\bigotimes \pi_{v}$.

If $\tau$ is any finite-dimensional representation of $W_{F_{v}}$, we say that $\tau$ is ramified if the restriction of $\tau$ to the inertia group in (1.4) is nontrivial. If $\tau$ is unramified, $\tau$ descends to a representation of the abelian group $\mathbb{Z}$ in (1.4). A representation of $\operatorname{Gal}\left(\bar{F}_{v} / F_{v}\right)$ yields a representation of $W_{F_{v}}$ by restriction, with the inertia group unchanged, and we may thus speak of ramification for a representation of $\operatorname{Gal}\left(\bar{F}_{v} / F_{v}\right)$.

We say that our given representation $\sigma$ of $\operatorname{Gal}(\bar{F} / F)$ is ramified at $v$ if $\sigma_{v}$ is ramified. The continuity of $\sigma$ implies that $\sigma$ descends to $\operatorname{Gal}(K / F)$ for some finite Galois extension $K$ of $F$. Almost every finite place of $F$ is unramified in $K$, and each of these places is a place where $\sigma_{v}$ is unramified. Therefore $\sigma$ is unramified at almost every place.

The statement of the conjecture gives us a finite set of places where $\pi$ is unramified but the $L$ functions of $\sigma$ and $\pi$ are not known to agree. We enlarge this set to a finite set $S$ so that $S$ contains all finite places where $\sigma$ or $\pi$ is ramified, as well as all infinite places. Since the Langlands and Godement-Jacquet $L$ factors of $\pi$ agree at all places $v \notin S$, we may write our given equality as

$$
L_{v}(s, \sigma)=L_{v}^{\mathrm{GJ}}(s, \pi) \quad \text { for } v \notin S,
$$

with an Artin $L$ factor on the left side and a Godement-Jacquet $L$ factor on the right side. The idea is to get information by twisting $\sigma$ and $\pi$ by suitable unitary Grossencharacters $\alpha$.

The twist $\sigma \otimes \alpha$ was discussed above. A little explanation is in order for $\pi \otimes \alpha$. The representation $\pi$ is an irreducible constituent of some $L^{2}\left(G L_{n}(F) \backslash G L_{n}(\mathbb{A})\right)_{\chi}$ generated by cuspidal functions. We can regard $\alpha$ as a representation of the center $Z(\mathbb{A})$ trivial on $Z(F)$, and then $\pi \otimes \alpha$ is an irreducible constituent of $L^{2}\left(G L_{n}(F) \backslash G L_{n}(\mathbb{A})\right)_{\chi \alpha}$ generated by cuspidal functions.

If $v$ is a finite place, let $\mathfrak{m}_{F_{v}}$ be the maximal ideal of $\mathcal{O}_{F_{v}}$. The conductor of a character $\beta$ of $F_{v}^{\times}$will be defined to be the integer $m \geq 0$ such that $\beta$ is trivial on $1+\mathfrak{m}_{F_{v}}^{m}$ but not on $1+\mathfrak{m}_{F_{v}}^{m-1}$. (For $m=0$, we understand the condition to be that $\beta$ is trivial on $\mathcal{O}_{F_{v}}^{\times}$; for $m=1$, we understand the condition to be that $\beta$ is trivial on $1+\mathfrak{m}_{F_{v}}$ but not on $\mathcal{O}_{F_{v}}^{\times}$.) By Corollary 2.5 we may regard $\beta$ as a character of $W_{F_{v}}^{\mathrm{ab}}$, hence as a one-dimensional representation of $W_{F_{v}}$. The condition that $m=0$ for $\beta$ is the same as the condition that this one-dimensional representation be trivial on 
the inertia group in (1.4); thus we may consistently define $\beta$ to be unramified if $m=0$.

Lemma 1. Under the assumption that $L_{v}(s, \sigma)=L_{v}^{\mathrm{GL}}(s, \pi)$ for $v \notin S$,

$$
L_{v}(s, \sigma \otimes \alpha)=L_{v}^{\mathrm{GJ}}(s, \pi \otimes \alpha) \quad \text { for } v \notin S
$$

for any Grossencharacter $\alpha$.

Proof. First suppose that $\alpha$ is unramified at $v$. Since $\sigma$ is unramified at $v$, (8.1) gives the formulas

$$
\begin{aligned}
L_{v}(s, \sigma) & =\operatorname{det}\left(1-\sigma_{v}(\mathrm{Fr}) q^{-s}\right)^{-1}, \\
L_{v}(s, \sigma \otimes \alpha) & =\operatorname{det}\left(1-\sigma_{v}(\mathrm{Fr}) \alpha_{v}\left(\varpi_{v}\right) q^{-s}\right)^{-1},
\end{aligned}
$$

where $\varpi_{v}$ is a member of $\mathcal{O}_{F_{v}}$ of module $q^{-1}$ and $\sigma_{v}(\mathrm{Fr})$ is some unitary matrix. If the eigenvalues of $\sigma_{v}(\mathrm{Fr})$ are $a_{1}, \ldots, a_{n}$, then

$$
\begin{gathered}
L_{v}(s, \sigma)=\prod_{j=1}^{n}\left(1-a_{j} q^{-s}\right)^{-1}, \\
L_{v}(s, \sigma \otimes \alpha)=\prod_{j=1}^{n}\left(1-a_{j} \alpha_{v}\left(\varpi_{v}\right) q^{-s}\right)^{-1} .
\end{gathered}
$$

Since $\pi$ is unramified at $v$, the discussion with (8.3) shows that $\pi_{v}$ is the Langlands subquotient of some unramified

$$
\operatorname{ind}_{T N}^{G L_{n}\left(F_{v}\right)}\left(\left(\chi_{1}, \ldots, \chi_{n}\right) \otimes 1\right)
$$

and that the associated $L$ factor is the factor for $\left(\rho_{\left(\chi_{1}, \ldots, \chi_{n}\right)}, 0\right)$. Thus

$$
L_{v}^{\mathrm{GJ}}(s, \pi)=\prod_{j=1}^{n}\left(1-b_{j} q^{-s}\right)^{-1},
$$

where $b_{j}=\chi_{j}\left(\varpi_{v}\right)$. The twist of (8.9) by $\alpha_{v}$ is the unramified

$$
\left.\operatorname{ind}_{T N}^{G L_{n}\left(F_{v}\right)}\left(\left(\chi_{1} \alpha_{v}, \ldots, \chi_{n} \alpha_{v}\right)\right) \otimes 1\right),
$$

and it follows from $[\mathrm{Ja1},(3.4)]$ that

$$
L_{v}^{\mathrm{GJ}}(s, \pi \otimes \alpha)=\prod_{j=1}^{n}\left(1-b_{j} \alpha_{v}\left(\varpi_{v}\right) q^{-s}\right)^{-1} .
$$

The assumed equality of (8.8a) and (8.10) forces every symmetric polynomial in $n$ variables to agree at $\left(a_{1}, \ldots, a_{n}\right)$ and $\left(b_{1}, \ldots, b_{n}\right)$, and hence we have an equality of unordered sets $\left\{a_{1}, \ldots, a_{n}\right\}=\left\{b_{1}, \ldots, b_{n}\right\}$. Therefore (8.8b) equals (8.12).

Now suppose that $\alpha$ is ramified at $v$. We shall show that $L_{v}(s, \sigma \otimes \alpha)$ and $L_{v}^{\mathrm{GJ}}(s, \pi \otimes \alpha)$ are both 1 . In the case of $\sigma$, decompose $\sigma_{v}$ into the direct sum of irreducibles $\tau$. Since $\sigma$ is unramified at $v$, each $\tau$ is unramified and thus $\tau \otimes \alpha_{v}$ is ramified. Let $V_{\tau}$ be the space on which $\tau$ acts. The invariant subspaces of $V_{\tau}$ under $\tau$ are the same as those under $\tau \otimes \alpha_{v}$, and the inertial invariants form a proper such subspace. By irreducibility the inertial invariants are 0 , and $L_{v}\left(s, \tau \otimes \alpha_{v}\right)=1$. Since $L_{v}(s, \sigma \otimes \alpha)$ is the product of such factors, $L_{v}(s, \sigma \otimes \alpha)=1$. 
In the case of $\pi$, we still have that $L_{v}^{\mathrm{GJ}}(s, \pi \otimes \alpha)$ is to be computed from (8.12) and that $L_{v}^{\mathrm{GJ}}(s, \pi \otimes \alpha)=\prod_{j=1}^{n} L_{v}^{\mathrm{GJ}}\left(s, \chi_{j} \alpha_{v}\right)$ by [Ja1, (3.4)]. But this time $\chi_{j} \alpha_{v}$ is one-dimensional and ramified. The subspace of invariants is $0, L_{v}^{\mathrm{GJ}}\left(s, \chi_{j} \alpha_{v}\right)$ is 1 , and $L_{v}^{\mathrm{GJ}}(s, \pi \otimes \alpha)=1$.

Lemma 2. Let $T$ and $T^{\prime}$ be finite sets of places, with $T$ containing only finite places. Fix integers $m_{u} \geq 0$ for $u \in T$. Then there exists a Grossencharacter $\alpha$ such that

(i) $\alpha_{v}=1$ for all $v$ in $T^{\prime}$ and

(ii) for each $u$ in $T$ the conductor of $\alpha_{u}$ is $\geq m_{u}$.

REFEREnCE. This is a special case of [Ar-Ta, Theorem 5, p. 103].

From the end of $\S 7$, we know that the cuspidal automorphic representation $\pi$ is unitary. The results of Flath [Fl] therefore imply that every local component $\pi_{v}$ is unitary. Although $\pi_{v}$ is not known to be tempered (see [Moe1] and [Moe2] for "tempered"), $\pi_{v}$ does satisfy another property - it is "generic" in a sense to be defined below.

Let $N$ be the algebraic group of matrices $x=\left(x_{i j}\right)$ with $x_{i j}$ equal to 0 for $i>j$, equal to 1 for $i=j$, and unrestricted for $i<j$. Fix a place $v$ and a nontrivial unitary character $\psi_{v}$ of the additive group $F_{v}$, and consider a unitary character $\theta_{v}$ of $N\left(F_{v}\right)$ of the form

$$
\theta_{v}(x)=\psi\left(c_{1} x_{12}+\cdots+c_{n-1} x_{n-1, n}\right) .
$$

We say that $\theta_{v}$ is nondegenerate if $c_{1} \cdots c_{n-1} \neq 0$. Let $\Pi^{(v)}$ be an irreducible unitary admissible representative of $G L_{n}\left(F_{v}\right)$, and let $V^{(v)}$ be the space of its underlying smooth representation (the space of $C^{\infty}$ vectors if $v$ is infinite, the space of vectors fixed by some idempotent of $\mathcal{H}_{v}$ if $v$ is finite). The representation $\Pi^{(v)}$ is said to be generic if there exists a nonzero continuous linear functional $\lambda$ on $V^{(v)}$ and some nondegenerate $\theta_{v}$ as above such that

$$
\lambda\left(\Pi^{(v)}(n g) x\right)=\theta_{v}(n) \lambda\left(\Pi^{(v)}(g) x\right) \quad \text { for all } n \in N\left(F_{v}\right), g \in G L_{n}\left(F_{v}\right), x \in V^{(v)} .
$$

Lemma 3. If $\Pi=\bigotimes_{v} \Pi_{v}$ is a cuspidal automorphic representation of $G L_{n}\left(\mathbb{A}_{F}\right)$, then each $\Pi_{v}$ is a unitary generic representation of $G L_{n}\left(F_{v}\right)$.

Reference. [Sha, Corollary, p. 190].

\section{Proof of Theorem 8.8.}

Step 1. If $v$ is any finite place, then there exists an integer $m=m(v, \sigma) \geq 0$ such that $L_{v}\left(s, \sigma_{v} \otimes \beta\right)=1$ for every unitary character $\beta$ of $F_{v}^{\times}$of conductor $\geq m$.

In fact, since the $L$ factor of a direct sum of representations is the product of the $L$ factors of the summands, it is enough to prove that the local factor $L_{v}(s, \tau \otimes \beta)$ is 1 for an irreducible $\tau$ when $m$ is large enough. Fix $\tau$.

If there exists $\beta_{0}$ with $\tau \otimes \beta_{0}$ unramified (at $v$ ), then $\tau \otimes \beta_{0}$ factors through the abelian group $\mathbb{Z}$ in (1.4), and $\tau$ must be one-dimensional. So we can regard $\tau$ as a character of $F_{v}^{\times}$, say with conductor $m_{0}$. If $\beta$ has conductor $>m_{0}$, then $\tau \otimes \beta$ will be ramified, and $L_{v}(s, \tau \otimes \beta)$ will be 1 .

Otherwise $\tau \otimes \beta$ is ramified (at $v$ ) for every unitary character $\beta$ of $F_{v}^{\times}$. Let $\tau$ act on $V_{\tau}$. The invariant subspaces of $V_{\tau}$ under $\tau$ are the same as under $\tau \otimes \beta$, and 
the inertial invariants form a proper such subspace. By irreducibility the inertial invariants are 0 , and therefore $L_{v}(s, \tau \otimes \beta)=1$.

Step 2. If $v$ is any finite place, then there exists an integer $m^{\prime}=m^{\prime}(v, \pi) \geq 0$ such that $L_{v}^{\mathrm{GJ}}\left(s, \pi_{v} \otimes \beta\right)=1$ for every unitary character $\beta$ of $F_{v}^{\times}$of conductor $\geq m^{\prime}$.

In fact, the Langlands classification ([Moe2]) shows that $\pi_{v}$ is the Langlands quotient of a representation induced from a parabolic subgroup with the product of a unitary discrete series and a quasicharacter on the Levi factor. The representation on the Levi factor is just the tensor product of similar representations on the component subgroups $G L_{n_{i}}\left(F_{v}\right)$, and [Ja1, (3.4)] shows that the $L$ factor of $\pi_{v}$ is the product of the $L$ factors of these representations on the component subgroups $G L_{n_{i}}\left(F_{v}\right)$. Thus it is enough to handle a representation of $G L_{k}\left(F_{v}\right)$ that is the product of a unitary discrete series and a quasicharacter. Work of Zelevinsky discussed in [Moe2] shows that such a representation $\eta$ is the unique irreducible submodule of an induced representation of the form

$$
\operatorname{ind}_{P}^{G L\left(k, F_{v}\right)}\left(\left((\mu \otimes \gamma) \otimes\left(|\cdot|^{\frac{k-1}{2}}, \ldots,|\cdot|^{-\frac{k-1}{2}}\right)\right) \otimes 1\right) .
$$

Here $P=M U$ is the standard parabolic subgroup associated to the partition $(d, d, \ldots, d)$ for some divisor $d$ of $n, M$ is the product of copies of $G L\left(d, F_{v}\right)$, and the representation $\mu \otimes \gamma$ of $M$ is the product of a (unitary) supercuspidal $\mu$ and a quasicharacter $\gamma$. By Theorem 8.2 of [Ja-P-S],

$$
L_{v}^{\mathrm{GJ}}(s, \eta \otimes \beta)=L_{v}^{\mathrm{GJ}}\left(s, \mu \otimes\left(\gamma \beta|\cdot|^{\frac{k}{d}-1}\right)\right)
$$

for any quasicharacter $\beta$ of $F_{v}^{\times}$. If $d>1$, then this $L$ factor is 1 for any $\beta$ by [Ja1, (1.3.5)]. Thus we may assume that $d=1$, in which case $\mu$ is just a unitary character of $F_{v}^{\times}$and the argument is finished as in the case of the Galois representation $\sigma$.

Step 3.

$$
L_{\infty}(s, \sigma) L_{\infty}^{\mathrm{GJ}}\left(1-s, \pi^{\vee}\right)=\varepsilon(s) L_{\infty}^{\mathrm{GJ}}(s, \pi) L_{\infty}\left(1-s, \sigma^{\vee}\right)
$$

for an entire nonvanishing function $\varepsilon(s)$.

In fact, let $T$ be the subset of finite places in $S$, and let $T^{\prime}$ be the subset of infinite places. For each $u$ in $T$, let $m_{u}$ denote the maximum of the numbers $m(u, \sigma), m\left(u, \sigma^{\vee}\right), m^{\prime}(u, \pi)$, and $m^{\prime}\left(u, \pi^{\vee}\right)$ given by Steps 1 and 2 . Let $\alpha$ be a Grossencharacter chosen by Lemma 2 for the data $T, T^{\prime}$, and $\left\{m_{u} \mid u \in T\right\}$. Then we have

$$
\begin{aligned}
L_{u}(s, \sigma \otimes \alpha) & =L_{u}^{\mathrm{GJ}}(s, \pi \otimes \alpha)=1 & & \text { for } u \in T \\
L_{v}(s, \sigma \otimes \alpha) & =L_{v}(s, \sigma) & & \text { for } v \in T^{\prime} \\
L_{v}^{\mathrm{GJ}}(s, \pi \otimes \alpha) & =L_{v}^{\mathrm{GJ}}(s, \pi) & & \text { for } v \in T^{\prime} .
\end{aligned}
$$

Similar formulas are valid for $\sigma^{\vee}$ and $\pi^{\vee}$. The global functional equations of $\Lambda(s, \sigma \otimes \alpha)$ and $\Lambda^{\mathrm{GJ}}(s, \pi \otimes \alpha)$ given in (5.4) (as generalized by Weil) and in Theorem 8.7 imply that

$$
\Lambda(s, \sigma \otimes \alpha) \Lambda^{\mathrm{GJ}}\left(1-s, \pi^{\vee} \otimes \alpha^{\vee}\right)=\varepsilon(s) \Lambda^{\mathrm{GJ}}(s, \pi \otimes \alpha) \Lambda\left(1-s, \sigma^{\vee} \otimes \alpha^{\vee}\right),
$$

where $\varepsilon(s)$ is entire and nonvanishing. Let $L^{S}$ or $L^{\mathrm{GJS}}$ denote a product of factors corresponding to the places not in $S$. Substituting from (8.14) into (8.15), we obtain

$$
\begin{aligned}
& L_{\infty}(s, \sigma) L^{S}(s, \sigma \otimes \alpha) L_{\infty}^{\mathrm{GJ}}\left(1-s, \pi^{\vee}\right) L^{\mathrm{GJ} S}\left(1-s, \pi^{\vee} \otimes \alpha^{\vee}\right) \\
& \quad=\varepsilon(s) L_{\infty}^{\mathrm{GJ}}(s, \pi) L^{\mathrm{GJ} S}(s, \pi \otimes \alpha) L_{\infty}\left(1-s, \sigma^{\vee}\right) L^{S}\left(1-s, \sigma^{\vee} \otimes \alpha^{\vee}\right) .
\end{aligned}
$$


Use of Lemma 1 allows us to cancel all the factors $L^{S}$ and $L^{\text {GJS }}$ in this formula, and we arrive at (8.13).

Step 4 .

$$
L_{\infty}(s, \sigma)=L_{\infty}^{\mathrm{GJ}}(s, \pi) \quad \text { and } \quad L_{\infty}\left(1-s, \sigma^{\vee}\right)=L_{\infty}^{\mathrm{GJ}}\left(1-s, \pi^{\vee}\right) .
$$

In fact, inspection of the formulas in [Kna2, (3.6) and (4.6)] shows for each infinite place $w$ that $L_{w}\left(s, \sigma_{w}\right)$ is nowhere vanishing and has no poles for $\operatorname{Re} s>0$. Similarly $L_{w}\left(1-s, \sigma_{w}^{\vee}\right)$ is nowhere vanishing and has no poles for $\operatorname{Re} s<0$.

We expect corresponding properties for $\pi$, but we get less. By [Ja1, (5.1)], each $L_{w}^{\mathrm{GJ}}\left(s, \pi_{w}\right)$ is an Artin $L$ factor and hence is nowhere vanishing. The conclusions about poles are more difficult to prove: $L_{w}\left(s, \pi_{w}\right)$ has no poles for $\operatorname{Re} s \geq \frac{1}{2}$, and $L_{w}\left(1-s, \pi_{w}^{\vee}\right)$ has no poles for $\operatorname{Re} s \leq \frac{1}{2}$. This result is stated as [Ba-R, Proposition 2.1]. Its proof combines the fact that $\pi_{w}$ is unitary and generic (as follows from Lemma 3) with a classication of (irreducible) unitary generic representations (which follows readily from Vogan's classification of irreducible unitary representations. (See [Moe2] for a qualitative discussion of Vogan's classification in this volume.)

If we rewrite $(8.13)$ as

$$
L_{\infty}(s, \sigma) / L_{\infty}^{\mathrm{GJ}}(s, \pi)=\varepsilon(s) L_{\infty}^{\mathrm{GJ}}\left(1-s, \sigma^{\vee}\right) / L_{\infty}^{\mathrm{GJ}}\left(1-s, \pi^{\vee}\right),
$$

then we see that the left side has no poles or zeros for $\operatorname{Re} s \geq \frac{1}{2}$ and the right side has no poles or zeros for $\operatorname{Re} s \leq \frac{1}{2}$. Therefore both sides are entire and nonvanishing.

To complete Step 4 , it is enough to show that a product of elementary $L$ factors for archimedean places is determined by its poles. Referring to [Kna2, (3.6) and (4.6)], we see that each such elementary $L$ factor is of the form $\pi^{-\frac{s}{2}-t} \Gamma\left(\frac{s}{2}+t\right)$ or $2(2 \pi)^{-s-u} \Gamma(s+u)$ for some $t$ or $u$. If we put $\Gamma_{\mathbb{R}}(s)=\pi^{-\frac{s}{2}} \Gamma\left(\frac{s}{2}\right)$, then we conclude from the well known formula $\Gamma\left(\frac{z}{2}\right) \Gamma\left(\frac{z}{2}+\frac{1}{2}\right)=\pi^{1 / 2} 2^{-z+1} \Gamma(z)$ that

$$
2(2 \pi)^{-s-u} \Gamma(s+u)=\Gamma_{\mathbb{R}}(s+u) \Gamma_{\mathbb{R}}(s+u+1) .
$$

Hence every product of elementary $L$ factors for archimedean places is of the form $\prod_{j=1}^{r} \Gamma_{\mathbb{R}}\left(s+t_{j}\right)$ for suitable $t_{j}$ 's. No factor has a zero, and the pole of $\Gamma_{\mathbb{R}}\left(s+t_{j}\right)$ with $\operatorname{Re} s$ largest occurs at $s=-t_{j}$ and has residue 2 . Thus we can decompose the product functions one factor at a time by finding the pole with Re $s$ as large as possible and dividing off a corresponding $\Gamma_{\mathbb{R}}\left(s+t_{j}\right)$.

Step 5. If $v$ is any finite place in $S$, then $L_{v}(s, \sigma)=L_{v}^{\mathrm{GJ}}(s, \pi)$.

In fact, let $T$ be the set of finite places in $S$ other than $v$, and let $T^{\prime}$ be the union of $\{v\}$ and the set of infinite places. For each $u$ in $T$, let $m_{u}$ denote the maximum of the numbers $m(u, \sigma), m\left(u, \sigma^{\vee}\right), m^{\prime}(u, \pi)$, and $m^{\prime}\left(u, \pi^{\vee}\right)$ given by Steps 1 and 2 . Let $\alpha$ be a Grossencharacter chosen by Lemma 2 for the data $T, T^{\prime}$, and $\left\{m_{u} \mid u \in T\right\}$. Arguing as in Step 3 and taking into account the result of Step 4, we are led to an equation

$$
L_{v}(s, \sigma) / L_{v}^{\mathrm{GJ}}(s, \pi)=\varepsilon_{1}(s) L_{v}^{\mathrm{GJ}}\left(1-s, \pi^{\vee}\right) / L_{v}^{\mathrm{GJ}}\left(1-s, \sigma^{\vee}\right) .
$$

where $\varepsilon_{1}(s)$ is an entire nonvanishing function.

Using the definition of $L_{v}\left(s, \sigma_{v}\right)$ in $(5.1 \mathrm{~b})$ and taking into account that $\sigma$ is unitary, we see that $L_{v}\left(s, \sigma_{v}\right)$ is a product of factors $\left(1-c q_{v}^{-s}\right)^{-1}$ with $|c|=1$. Hence $L_{v}\left(s, \sigma_{v}\right)$ is nowhere vanishing and has no poles for $\operatorname{Re} s>0$. Similarly $L_{v}\left(1-s, \sigma^{\vee}\right)$ is nowhere vanishing and has no poles for $\operatorname{Re} s<1$. 
Again we expect corresponding formulas for $\pi$, but matters are not so simple. The conclusions are that $L_{v}^{\mathrm{GJ}}(s, \pi)$ has no poles for $\operatorname{Re} s \geq \frac{1}{2}$ and $L_{v}^{\mathrm{GJ}}\left(1-s, \pi^{\vee}\right)$ has no poles for $\operatorname{Re} s \leq \frac{1}{2}$, and again the result is stated as [Ba-R, Proposition 2.1]. Its proof uses Lemma 3 and a classification of the irreducible unitary generic representations. (A somewhat different proof may be found in [Ja-P-S].)

Arguing as in Step 4, we write (8.16) as

$$
L_{v}(s, \sigma) / L_{v}^{\mathrm{GJ}}(s, \pi)=\varepsilon_{1}(s) L_{v}^{\mathrm{GJ}}\left(1-s, \sigma^{\vee}\right) / L_{v}^{\mathrm{GJ}}\left(1-s, \pi^{\vee}\right) .
$$

The restrictions on zeros and poles imply that each side is entire and nonvanishing. The left side is of the form $\left(1+P\left(q_{v}^{-s}\right)\right) /\left(1+Q\left(q_{v}^{-s}\right)\right)$, where $P$ and $Q$ are polynomials without constant term. For this expression to have neither poles nor zeros, we must have $P=Q$. Therefore $L_{v}(s, \sigma)=L_{v}^{\mathrm{GJ}}(s, \pi)$. This completes Step 5 and the proof of Theorem 8.8.

\section{9. $L$ Groups and General Langlands $L$ Functions}

With $G L_{n}$ in place as a model, we can now describe what the Langlands theory proposes for an arbitrary linear reductive group $G$ over a number field $F$. It will be assumed throughout that $G(\mathbb{C})$ is connected.

The material in this section is largely due to Langlands [Lgl2] and [Lgl3], and the presentation amounts to a summary of the exposition [Bo4]. The reader may wish to consult [Bo4] for a more precise and detailed account and [Bl-Ro] for a discussion that includes a number of examples.

The theory introduces a group ${ }^{L} G$ that is the semidirect product of a certain complex reductive group $\widehat{G}$ and the Galois group $\operatorname{Gal}(\bar{F} / F)$, with $\widehat{G}$ normal. ${ }^{5}$

When $G=G L_{n}, \widehat{G}$ is $G L_{n}(\mathbb{C})$, and the semidirect product is a direct product; the definitions will show that the Galois group can often be ignored in this case, and we are reduced to the situation in $\S 8$.

The same construction as for the $L$ group of $G(F)$ yields, for each place $v$ of $F$, an $L$ group for $G\left(F_{v}\right)$. The complex group $\widehat{G}$ is unchanged, and the Galois group $\operatorname{Gal}(\bar{F} / F)$ is cut down to the decomposition subgroup $\operatorname{Gal}\left(\bar{F}_{v} / F_{v}\right)$.

Thinking in terms of $F_{v}$, let us consider the case of an arbitrary local field $k$ of characteristic 0 containing $F$. Then we can form an $L$ group for $G(k)$. An "admissible homomorphism" is a certain kind of homomorphism $\varphi$ (to be described below) of the Weil group $W_{k}$ or the Weil-Deligne group $W_{k}^{\prime}$, according as $k$ is archimedean or nonarchimedean, into the $L$ group of $G(k)$ that "covers the identity mapping on $\operatorname{Gal}(\bar{k} / k)$." Here " $\varphi$ covers the identity mapping on $\operatorname{Gal}(\bar{k} / k)$ " means that when $\varphi$ is followed by the map of the $L$ group to $\operatorname{Gal}(\bar{k} / k)$, the result is the usual map of $W_{k}$ or $W_{k}^{\prime}$ into $\operatorname{Gal}(\bar{k} / k)$. Two admissible homomorphisms are equivalent if they are conjugate via $\widehat{G}$, and the set of equivalence classes is denoted $\Phi(G(k))$.

We associate an elementary $L$ factor $L(s, \varphi, r)$ to this situation whenever $r$ is a representation of ${ }^{L} G$ into some $G L_{n}(\mathbb{C})$ that is holomorphic in the $\widehat{G}$ variable. The

\footnotetext{
${ }^{5}$ To handle more advanced topics such as endoscopy, some adjustment in the definition of ${ }^{L} G$ is needed. The traditional adjustment is to replace the Galois group by a Weil group. Another possibility, discussed in [Ad-Ba-V], is to use an extension of $\widehat{G}$ by the Galois group that is not necessarily a semidirect product.
} 
definition is simply

$$
L(s, \varphi, r)=L(s, r \circ \varphi),
$$

where the right side is given by (8.1). When $G$ is $G L_{n}$ and $r$ is the standard representation, this definition reduces to the definition (8.1) for the group $G L_{n}$.

As with $G L_{n}$, we let $\Pi(G(k))$ be the set of equivalence classes of irreducible admissible representations of $G(k)$.

Local Langlands Conjecture. $\Pi(G(k))$ is partitioned in a natural way into finite nonempty subsets $\Pi_{\varphi}$ indexed by all $\varphi \in \Phi(G(k))$.

As in the case of $G L_{n}$, the correspondence is to be consistent with a number of conditions. See [Bo4, p. 43] for details. Ideally the correspondence should be consistent with functoriality, which we shall define in $\S 10$. Consistency with functoriality implies that the sets $\Pi_{\varphi}$ are not necessarily singleton sets, unlike the case of $G L_{n}$. (See $\S 10$ below.) A set $\Pi_{\varphi}$ is called an $L$ packet, and members of the same $\Pi_{\varphi}$ are said to be $L$ indistinguishable.

The conjecture is known to be true if $G$ is a torus ([Lgl1], summarized in [Bo4, p. 41]) and if $k$ is archimedean ([Lgl3], summarized in [Bo4, p. 46]). Cases with $G=G L_{n}$ for which it is true were discussed in $\S 8$. Also we shall insist that representations with a nonzero $G\left(\mathcal{O}_{k}\right)$ fixed vector (i.e., the Langlands subquotients of the unramified principal series) be parametrized by $\varphi$ 's in a particular way; this parametrization we shall discuss below.

The Local Langlands Conjecture allows us to define an elementary $L$ factor whenever $\pi$ is an irreducible admissible representation of $G(k)$ and $r$ is a finitedimensional holomorphic representation of ${ }^{L} G$. The definition is simply

$$
L(s, \pi, r)=L(s, \varphi, r) \quad \text { if } \varphi \leftrightarrow \pi,
$$

with $L(s, \varphi, r)$ as in (9.1). When $G$ is $G L_{n}$ and $r$ is the standard representation, this definition reduces to the definition (8.2) for $G L_{n}$.

Before continuing, let us fill in some details in the above discussion.

We begin with a rough description of the $L$ group ${ }^{L} G$. The group $\widehat{G}$ is a connected complex reductive group, having the same dimension as $G$ and having root system equal to the system of coroots $2 \alpha /\langle\alpha, \alpha\rangle$ of $G$. The question of the exact size of the center is somewhat involved and will be described in a moment. For our purposes, it will be enough to know that

(i) if $G=G L_{n}$, then $\widehat{G}=G L_{n}(\mathbb{C})$

(ii) if $G(\mathbb{C})$ is simply connected, then $\widehat{G}$ is an adjoint group

(iii) if $G(\mathbb{C})$ is an adjoint group, then $\widehat{G}$ is simply connected.

Here are some examples:

\begin{tabular}{|c|c|}
\hline$G$ & $\widehat{G}$ \\
\hline$S L_{n}$ & $P G L_{n}(\mathbb{C})$ \\
$P G L_{n}$ & $S L_{n}(\mathbb{C})$ \\
$S p_{2 n}$ & $S O_{2 n+1}(\mathbb{C})$ \\
$S O_{2 n+1}$ & $S p_{2 n}(\mathbb{C})$ \\
\hline
\end{tabular}

Langlands [Lgl3] captured the exact size of the center of $\widehat{G}$ by working with weight lattices, but the treatment in [B4] makes matters axiomatic with the 
notion of root datum, which is described in [Sp]. A root datum is a 4-tuple $\left(X, \Delta, X^{\vee}, \Delta^{\vee}\right)$, where

(i) $X$ and $X^{\vee}$ are free abelian groups of finite rank in duality by a pairing $X \times X^{\vee} \rightarrow \mathbb{Z}$ denoted $\langle\cdot, \cdot\rangle$,

(ii) $\Delta$ and $\Delta^{\vee}$ are reduced root systems lying in subspaces of $X \subset X \otimes \mathbb{R}$ and $X^{\vee} \subset X^{\vee} \otimes \mathbb{R}$, respectively,

(iii) $\Delta$ and $\Delta^{\vee}$ are in bijection by a map $\alpha \mapsto \alpha^{\vee}$ such that $\left\langle\alpha, \alpha^{\vee}\right\rangle=2$ for all $\alpha \in \Delta$,

(iv) $\Delta$ is preserved by the maps $s_{\alpha}: X \rightarrow X$ given by $s_{\alpha}(x)=x-\left\langle x, \alpha^{\vee}\right\rangle \alpha$, and

(v) $\Delta^{\vee}$ is preserved by the maps $s_{\alpha} \vee: X^{\vee} \rightarrow X^{\vee}$ given by $s_{\alpha} \vee(x)=$ $x-\langle x, \alpha\rangle \alpha^{\vee}$.

To our reductive group $G$ and a maximal torus $T$ is associated a root datum $\psi(G, T)=\left(X, \Delta, X^{\vee}, \Delta^{\vee}\right)$ as follows: $X$ is the group of rational characters $X^{*}(T)$ (i.e., algebraic homomorphisms $\left.T \rightarrow G L_{1}\right), X^{\vee}$ is the group $X_{*}(T)$ of oneparameter subgroups (i.e., algebraic homomorphisms $G L_{1} \rightarrow T$ ), the form $\langle x, u\rangle$ for $x \in X^{*}(T)$ and $u \in X_{*}(T)$ is given by $x(u(t))=t^{\langle x, u\rangle}$ for all $t \in \bar{F}^{\times}, \Delta$ is the root system of $(G, T)$, and $\Delta^{\vee}$ is the system of coroots described as a subset of $X_{*}(T)$ in [Sp, pp. 6-7]. The theorem below says that all root data arise in this way.

Theorem 9.1. For any root datum $\Psi$, there exists a connected reductive group $G$ and a maximal torus $T$ in $G$ such that $\Psi=\psi(G, T)$. The pair $(G, T)$ is unique up to isomorphism over $\bar{F}$.

REFERENCE. [Sp, p. 9].

If $\Psi=\left(X, \Delta, X^{\vee}, \Delta^{\vee}\right)$ is a root datum, then $\Psi^{\vee}=\left(X^{\vee}, \Delta^{\vee}, X, \Delta\right)$ is easily seen to be a root datum. Starting from $(G, T)$, we assemble the root datum $\psi(G, T)$ and apply Theorem 9.1 to $\psi(G, T)^{\vee}$. The group $\widehat{G}$ is defined to be the $\mathbb{C}$ points of the reductive group produced by the theorem. Also we define $\widehat{T}$ to be the $\mathbb{C}$ points of the maximal torus produced by the theorem.

All this discussion really involved only groups defined over $\bar{F}$. We bring in $F$ through an action of $\operatorname{Gal}(\bar{F} / F)$. Fix a positive system $\Delta^{+}$. If $\gamma$ is in $\operatorname{Gal}(\bar{F} / F)$, then $\gamma$ carries $T(\bar{F})$ to $T(\bar{F})$ and $\Delta$ to $\Delta$, and there exists a member $g_{\gamma}$ of $G(\bar{F})$ that normalizes $T(\bar{F})$ such that $g_{\gamma} \gamma$ carries $\Delta^{+}$to itself. Any two such elements $g_{\gamma}$ are in the same coset relative to $T(\bar{F})$, and thus we can associate to $\gamma$ a unique permutation of $\Delta^{+}$. This composite element also acts as an automorphism of $X^{*}(T)$ and $X_{*}(T)$. As $\gamma$ varies, we obtain compatible group actions of $\mathrm{Gal}(\bar{F} / F)$ on $\Delta^{+}$, $X^{*}(T)$, and $X_{*}(T)$. Thus we obtain a group action of $\operatorname{Gal}(\bar{F} / F)$ on the dual root datum $\left(X_{*}(T), \Delta^{\vee}, X^{*}(T), \Delta\right)$ preserving positive roots. This action lifts uniquely to an action of $\operatorname{Gal}(\bar{F} / F)$ on $\widehat{G}$ once we choose root vectors for the simple roots. Taking all the choices into account, we find that this action is canonical up to inner automorphism by a member of $\widehat{T}$. In any event, ${ }^{L} G$ is defined to be the semidirect product of $\operatorname{Gal}(\bar{F} / F)$ and $\widehat{G}$. Henceforth we shall usually discuss only groups $G$ that are split over $F$; then the action is trivial, and ${ }^{L} G$ is the direct product and $\widehat{G}$ and the Galois group.

Let us now return to $G(k)$ with $k$ a local field of characteristic 0 that contains $F$. We shall define admissible homomorphisms more precisely, but only in the case that 
$G(k)$ is quasisplit over $k$. An element of ${ }^{L} G$ is said to be semisimple or unipotent according as its $\widehat{G}$ component is semisimple or unipotent. If $k$ is nonarchimedean, an admissible homomorphism is a continuous homomorphism $\varphi$ of the WeilDeligne group $W_{k}^{\prime}$ into ${ }^{L} G$ with the following properties:

(i) $\varphi$ covers the identity mapping of $\operatorname{Gal}(\bar{k} / k)$,

(ii) $\varphi$ is holomorphic in the $\mathbb{C}$ variable of $W_{k}^{\prime}$, and every member of $\varphi(\mathbb{C})$ is unipotent, and

(iii) every member of $\varphi\left(W_{k}\right)$ is semisimple.

(Another condition, involving "relevance" of parabolic subgroups, is needed if $G(k)$ is not quasisplit. See [Bo4, p. 40].) If $k$ is archimedean, admissible homomorphisms are defined in the same way except that $W_{k}$ replaces $W_{k}^{\prime}$ and condition (ii) is dropped.

The definitions are now complete in the case of $G(k)$, and the Local Langlands Conjecture allows us to associate to each irreducible admissible representation $\pi$ of $G(k)$ and holomorphic representation $r$ of ${ }^{L} G$ an elementary $L$ factor $L(s, \pi, r)$.

It is known what these $L$ factors should be in the case of unramified principal series. To keep matters simple, let us suppose that $G$ is split over the nonarchimedean field $k$. Let $Q$ be the compact subgroup $Q=G\left(\mathcal{O}_{k}\right)$. We shall assume that $Q$ is a "hyperspecial maximal compact subgroup" in the sense of the exposition [ $\mathrm{Ti}$, pp. 35, 55]. Examples where this condition is satisfied are where $G\left(\mathcal{O}_{k}\right)$ is $G L_{n}\left(\mathcal{O}_{k}\right)$ in $G L_{n}(k), S p_{n}\left(\mathcal{O}_{k}\right)$ in $S p_{n}(k)$, or $S O_{n}\left(\mathcal{O}_{k}\right)$ in $S O(k)$; here $S O_{n}$ is the group of matrices of determinant one preserving a quadratic form

$$
x_{1} x_{p+1}+\cdots x_{p} x_{2 p}+x_{2 p+1}^{2}
$$

in which $p=\left[\frac{n}{2}\right]$ and the last term is absent if $n$ is even. See [Car, p. 140] and [Bo4, pp. 38-39, 45] for further discussion of the terms "special" and "hyperspecial."

Suppose that $T$ is a $k$ split maximal torus chosen so that $T(k) \cap Q$ is maximal compact in $T(k)$. Let $\mathcal{H}(G(k), Q)$ be the subalgebra of bi- $Q$-invariant functions in the Hecke algebra of $G(k)$. This subalgebra is known to be commutative, and the characteristic function of $Q$ is the identity. Choose a Borel subalgebra $B=T N$ containing $T$, normalize Haar measure $d m$ on $N(k)$ so that $N \cap Q$ gets total measure 1 , and let $\Delta(t)$ be the positive function on $T(k)$ defined by $\Delta(t)=d\left(t n t^{-1}\right) / d n$. For $f \in \mathcal{H}(G(k), Q)$, define

$$
S f(t)=\Delta(t)^{1 / 2} \int_{N(k)} f(t n) d n \quad \text { for } t \in T(k) .
$$

Theorem 9.2. With the above assumptions and notation, the mapping $S$ is an algebra isomorphism of $\mathcal{H}(G(k), Q)$ onto the subalgebra $\mathcal{H}(T(k), T(k) \cap Q)^{W}$ of Weyl-group invariants in $\mathcal{H}(T(k), T(k) \cap Q)$.

Reference. This theorem is due to Satake, and the mapping $S$ is called the Satake isomorphism. See [Car, p. 147].

Now let $\pi$ be an irreducible admissible representation with a nonzero vector $\phi$ fixed by $Q$. The space of all vectors fixed by $Q$ is one-dimensional, and it follows that $\pi(\mathcal{H}(G(k), Q))$ operates on $\phi$ by scalars. The result is an algebra homomorphism $\lambda=\lambda(\pi)$ of $\mathcal{H}(G(k), Q)$ into $\mathbb{C}$. Sorting out the notation with the aid of [Car, p. 134], we see that the algebra homomorphisms of $\mathcal{H}(T(k), T(k) \cap Q)$ into $\mathbb{C}$ are simply the members of $\widehat{T}$, and it follows that we may identify an algebra 
homomorphism of $\mathcal{H}(T(k), T(k) \cap Q)^{W}$ into $\mathbb{C}$ with a $W$ orbit in $\widehat{T}$. Thus Theorem 9.2 implies that we may regard $\lambda(\pi)$ as a $W$ orbit in $\widehat{T}$.

We may understand this construction in other terms as follows. The Hecke algebra $\mathcal{H}(T(k), T(k) \cap Q)$ is just the $\mathbb{C}$ group ring of $\widehat{T}$, which we write as $\mathbb{C}[\widehat{T}]$. The subspace $\mathbb{C}[\widehat{T}]^{W}$ of Weyl-group invariants is the coordinate ring of the affine variety $\widehat{T} / W$, and then it becomes clear that a homomorphism of this algebra into $\mathbb{C}$ is simply a $W$ orbit in $\widehat{T}$. If $\pi$, as above, is an irreducible admissible representation of $G(k)$ with a nonzero vector fixed by $Q$, then the point it defines in $\widehat{T} / W$ is called the Langlands class of $\pi$. For $G=G L_{n}$, this point can be represented by an $n$-tuple $\left(\varepsilon_{1}, \ldots, \varepsilon_{n}\right)$ up to permutation, and the local $L$ factor that we used in $\S 8$ amounts to $L(s, \pi)=\prod_{i=1}^{n}\left(1-\varepsilon_{i} q^{-s}\right)^{-1}$ in these terms.

In the general case that we are studying, the elementary $L$ factor associated to $\pi$ and a holomorphic representation $r$ of ${ }^{L} G$ is taken to be

$$
L(s, \pi, r)=\operatorname{det}\left(1-r(\lambda(\pi)) q^{-s}\right)^{-1} .
$$

(See [Bo4, p. 39].) In (9.3) any representative of the $W$ orbit $\lambda(\pi)$ in $\widehat{T}$ is to be used, and the determinant is independent of the choice. To arrange for this $L$ factor to come from an admissible homomorphism $\varphi$, choose Fr in $W_{k}$ with $\|\mathrm{Fr}\|=q^{-1}$, and fix a representative of $\lambda(\pi)$ in $\widehat{T}$. The idea is to make $\varphi(\operatorname{Fr})=(\lambda(\pi), \operatorname{Fr})$ and to make $\varphi$ behave in an "unramified" fashion. To do so, define $\varepsilon: W_{k}^{\prime} \rightarrow \mathbb{Z}$ by

$$
\|\operatorname{Fr}\|^{\varepsilon(z, w)}=\|w\| \quad \text { for } z \in \mathbb{C} \text { and } w \in W_{k},
$$

and let

$$
\varphi(z, w)=(\lambda(\pi), \operatorname{Fr})^{\varepsilon(z, w)} .
$$

With this $\varphi$ associated to $\pi$, the definition (9.2) attaches (9.3) to $\pi$ and $r$ as elementary $L$ factor.

When $G(k)$ is not $k$ split, the treatment of unramified principal series involves considerably more structure theory, and the Satake isomorphism is more complicated to state. For an exposition of the structure theory, see [Ti] and [Car]. For the definition of the elementary $L$ factor associated to an irreducible admissible representation with a nonzero fixed vector under $G\left(\mathcal{O}_{k}\right)$, see [Bo4, pp. 39 and $44-45]$.

Let us return to the number field $F$. As was true for $G L_{n}$, the results of [Fl] show that an irreducible admissible representation $\pi$ of $G\left(\mathbb{A}_{F}\right)$ is a restricted tensor product $\pi=\bigotimes_{v} \pi_{v}$, and moreover almost every $\pi_{v}$ has a nonzero vector fixed under $G\left(\mathcal{O}_{v}\right)$. Let $r$ be a holomorphic representation of the $L$ group of $G(F)$. Since the $L$ group of $G\left(F_{v}\right)$ may be taken to be a subgroup of $G(F)$, we obtain by restriction a holomorphic representation $r$ of the $L$ group of each $G\left(F_{v}\right)$. For the irreducible admissible representation $\pi=\bigotimes_{v} \pi_{v}$ of $G\left(\mathbb{A}_{F}\right)$, we can then define

$$
\begin{aligned}
& L(s, \pi, r)=\prod_{\text {finite } v} L\left(s, \pi_{v}, r\right) \\
& \Lambda(s, \pi, r)=\prod_{\text {all } v} L\left(s, \pi_{v}, r\right),
\end{aligned}
$$

with $L\left(s, \pi_{v}, r\right)$ as in (9.2) (and almost always as in (9.3)). 
Theorem 9.3. If $\pi$ is an automorphic representation of $G\left(\mathbb{A}_{F}\right)$ and $r$ is a holomorphic representation of ${ }^{L} G$, then $L(s, \pi, r)$ converges absolutely for $\operatorname{Re} s$ sufficiently large.

REFEREnCE. This theorem is due to Langlands. The line of argument was given in Corollary 8.2 through Corollary 8.6.

The global analytic properties of $\Lambda(s, \pi, r)$, if any, are known only in special cases. It is expected that these functions are meromorphic and satisfy a functional equation. For more information, see [Bo4].

\section{Functoriality}

Functoriality refers to translating knowledge of a holomorphic homomorphism of $L$ groups into results about automorphic representations. Much of [Lgl2] raises specific questions about this problem, and [Bo4, $\S \S 15-17]$ discusses progress as of the late 1970s. More recent progress is the subject of [Lgl7].

A homomorphism $\omega: G \rightarrow H$ between reductive Lie groups over a local or global field induces a holomorphic homomorphism $\psi:{ }^{L} H \rightarrow{ }^{L} G$ if the image of $\omega$ is normal [Bo4, p. 29], and moreover $\psi$ covers the identity mapping of the Galois group. For example, the inclusion $\omega$ of $S L_{2}(\mathbb{R})$ into $G L_{2}(\mathbb{R})$ induces the natural quotient map

$$
\psi: G L_{2}(\mathbb{C}) \times \Gamma \rightarrow P G L_{2}(\mathbb{C}) \times \Gamma
$$

of $L$ groups, where $\Gamma=\operatorname{Gal}(\mathbb{C} / \mathbb{R})$. An admissible homomorphism

$$
\varphi: W_{\mathbb{R}} \rightarrow G L_{2}(\mathbb{C}) \times \Gamma
$$

induces by composition the admissible homomorphism

$$
\psi \circ \varphi: W_{\mathbb{R}} \rightarrow P G L_{2}(\mathbb{C}) .
$$

The resulting map $\Phi\left(G L_{2}(\mathbb{R})\right) \rightarrow \Phi\left(S L_{2}(\mathbb{R})\right.$ ) gives us a correspondence (not a function!) $\Pi\left(G L_{2}(\mathbb{R})\right) \rightarrow \Pi\left(S L_{2}(\mathbb{R})\right)$ since the Local Langlands Conjecture is a theorem in the archimedean case. Examining matters, we see that we associate to each irreducible admissible representation of $G L_{2}(\mathbb{R})$ all of its constituents on restriction to $S L_{2}(\mathbb{R})$. Nothing very deep is happening here, but the fact that each discrete series of $G L_{2}(\mathbb{R})$ and certain principal series decompose into two inequivalent pieces on restriction to $S L_{2}(\mathbb{R})$ forces some members of $\Pi\left(S L_{2}(\mathbb{R})\right)$ to have more than one element.

Let us call a holomorphic homomorphism $\psi:{ }^{L} H \rightarrow{ }^{L} G$ covering the identity of the Galois group an $L$ homomorphism. Not every $L$ homomorphism arises from a homomorphism $G \rightarrow H$; in fact, most do not. We can still ask

(a) in the local case whether such a $\psi$ induces a correspondence $\Pi(H) \rightarrow \Pi(G)$ and

(b) in the global case whether such a $\psi$ induces a correspondence of automorphic representations to automorphic representations (or cuspidal representations to cuspidal representations).

In each case we ask that the correspondence respect $L$ functions, or as much of 
$L$ functions as is known. (Also we ask that the correspondence respect $\varepsilon$ factors, which we have largely ignored in this article.)

To fix the ideas, let us continue with the notation of $\S 9$. Thus let $F$ be a number field, and let $G$ be a reductive group over $F$. We denote by $k$ any local field containing $F$, so that $G(k)$ is defined.

First let us consider the local case. If we accept the Local Langlands Conjecture, then (a) is solved, by the same argument as in the example above: The $L$ homomorphism $\psi:{ }^{L} H \rightarrow{ }^{L} G$ induces a map $\psi_{*}: \Phi(H) \rightarrow \Phi(G)$ (at least if $G$ is quasisplit, so that the all the conditions on a member of $\Phi(G)$ were given in $\S 9$ ). In turn, $\psi_{*}$ induces a correspondence $\Pi(H) \rightarrow \Pi(G)$, and the correspondence respects $L$ factors.

From an organizational point of view, we could insist that the Local Langlands Conjecture is to be proved first (or else taken as a working hypothesis), and then functoriality is to be addressed. But [Bo4] points out some early cases in which a partial result about local functoriality was established and then used to obtain a partial result about the Local Langlands Conjecture. If local functoriality and the Local Langlands Conjecture ultimately turn out to be true, the proofs may therefore have to start from some basic information (the $\varphi$ 's for the unramified principal series, the $L$ and $\varepsilon$ factors for $G L_{n}$ and the standard representation, and some other conditions) and establish local functoriality and the Local Langlands Conjecture together.

Nevertheless, to keep matters brief, we shall take local functoriality as a working hypothesis and consider the global case (b). Here is one possible statement of the problem.

Question (Global functoriality). Let $G$ and $H$ be reductive groups over $F$ with $G$ quasisplit, and let $\psi:{ }^{L} H \rightarrow{ }^{L} G$ be an $L$ homomorphism. For each place $v$ of $F$, let $\psi_{v}$ be the restriction of $\psi$ to a map between the $L$ groups of $H\left(F_{v}\right)$ and $G\left(F_{v}\right)$, let $\left(\psi_{v}\right)_{*}: \Phi\left(H\left(F_{v}\right)\right) \rightarrow \Phi\left(G\left(F_{v}\right)\right)$ be the induced map on admissible homomorphisms, and let $\left(\psi_{v}\right)_{*}$ also denote the correspondence $\Pi\left(H\left(F_{v}\right)\right) \rightarrow \Pi\left(G\left(F_{v}\right)\right)$ obtained from the Local Langlands Conjecture. Let $\pi=\bigotimes_{v} \pi_{v}$ be an automorphic representation of $H\left(\mathbb{A}_{F}\right)$.

(i) Does there exists a choice $\Pi_{v} \in\left(\psi_{v}\right)_{*}\left(\pi_{v}\right)$ for every $v$ such that $\Pi=\bigotimes_{v} \Pi_{v}$ is an automorphic representation of $G\left(\mathbb{A}_{F}\right)$ ?

(ii) If so, and if $\pi$ is cuspidal, under what conditions is $\Pi$ cuspidal?

This is an extremely deep question, even if the condition $\Pi_{v} \in\left(\psi_{v}\right)_{*}\left(\pi_{v}\right)$ is required only at almost every place. (Relaxing the requirement in this way allows one to address the question without first establishing the Local Langlands Conjecture.) Here are two illustrations, taken from [Lgl2], of just how deep it is. Let us write $\Gamma=\operatorname{Gal}(\bar{F} / F)$ and $\Gamma_{v}=\operatorname{Gal}\left(\bar{F}_{v} / F_{v}\right)$ with $\Gamma_{v} \subset \Gamma$.

Example 1. Let $G=G L_{n}$ and $H=\{1\}$, so that ${ }^{L} H=\{1\} \times \Gamma$ and ${ }^{L} G=$ $G L_{n}(\mathbb{C}) \times \Gamma$. Fix an $n$-dimensional representation $\sigma$ of $\Gamma$. The map $\psi:{ }^{L} H \rightarrow{ }^{L} G$ given by $\psi(1, \gamma)=(\sigma(\gamma), \gamma)$ is an $L$ homomorphism. If $v$ is a finite place of $F$, then the only admissible homomorphism for $H\left(F_{v}\right)$ is $\varphi_{0}^{(v)}(z, w)=(1, \iota(w))$, where $\iota: W_{F_{v}} \rightarrow \Gamma_{v}$ is the inclusion. Let $r$ be the representation $1 \times \sigma$ of ${ }^{L} H$, and let $p: W_{k}^{\prime} \rightarrow W_{k}$ be the natural quotient map. Then the elementary $L$ factor for the 
representation $r$ at the finite place $v$ is

$$
\begin{aligned}
L(s, 1, r) & =L\left(s, r \circ \varphi_{0}^{(v)}\right) & & \text { by }(9.1) \\
& =L(s, \sigma \circ \iota \circ p) & & \\
& =\operatorname{det}\left(1-\left(\left.\sigma \circ \iota(\mathrm{Fr})\right|_{\left(\mathbb{C}^{n}\right)^{I}}\right) q^{-s}\right)^{-1} & & \text { by }(8.1),
\end{aligned}
$$

and the right side coincides with the Artin $L$ factor (5.1) for $r$. Hence the global $L$ function for the cuspidal representation 1 of $H\left(\mathbb{A}_{F}\right)=\{1\}$ and for the representation $r$ coincides with the Artin $L$ function of $r$. If the answer to (i) is affirmative, let $\Pi \in \psi_{*}(1)$ be an automorphic representation of $G L_{n}\left(\mathbb{A}_{F}\right)$ for which $\Pi_{v} \in\left(\psi_{v}\right)_{*}\left(1_{v}\right)$ for each $v$. The admissible homomorphism $\Phi_{v}$ for $\Pi_{v}$ has $\Phi_{v}=\left(\psi_{v}\right)_{*}\left(\varphi_{0}^{(v)}\right)=$ $\psi_{v} \circ \varphi_{0}^{(v)}$. Let $R$ be the standard representation of ${ }^{L} G$ (trivial on $\Gamma$ ). Then we have

$$
\begin{aligned}
L\left(s, \Pi_{v}\right)=L\left(s, \Pi_{v}, R\right) & =L\left(s, \psi_{v} \circ \varphi_{0}^{(v)}, R\right) & & \text { by }(9.2) \\
& =L\left(s, R \circ \psi_{v} \circ \varphi_{0}^{(v)}\right) & & \text { by }(9.1) \\
& =L(s, \sigma \circ \iota \circ p) & & \\
& =L(s, 1, r) & & \text { by }(10.1) .
\end{aligned}
$$

Thus an affirmative answer to (i) for this situation implies that the Artin $L$ function for any $n$-dimensional representation of $\Gamma$ is the $L$ function of an automorphic representation of $G L_{n}$. An affirmative answer to (ii) implies that the latter representation is cuspidal, i.e., gives an affirmative answer to the Langlands Reciprocity Conjecture of $\S 8$.

Example 2. Let $H$ be general, and let $r$ be a holomorphic representation of ${ }^{L} H$ into $G L_{n}(\mathbb{C})$. Put $G=G L_{n}$, and define $\psi(x, \gamma)=(r(x, \gamma), \gamma)$. Let $\pi$ be an automorphic representation of $H$. If the answer to (i) is affirmative, let $\Pi \in \psi_{*}(\pi)$ be a corresponding automorphic representation of $G L_{n}\left(\mathbb{A}_{F}\right)$. Tracking down the definitions as in Example 1, we find that

$$
L(s, \pi, r)=L(s, \Pi),
$$

i.e., the $L$ function of $\pi$ and the representation $r$ is a standard $L$ function for $G L_{n}$. If we assume about $G L_{n}$ that Langlands $L$ functions and Godement-Jacquet $L$ functions coincide, then it follows from a generalization of Theorem 8.7 proved in [Ja1] that $\Lambda(s, \pi, r)$ has a meromorphic continuation to $\mathbb{C}$ and satisfies a functional equation. Moreover if $\pi$ is cuspidal and the answer to (ii) is affirmative, then (with some exceptions that can be sorted out) $L(s, \pi, r)$ and $\Lambda(s, \pi, r)$ are entire.

We mention two situations in which substantial progress has been made in establishing global functoriality. Both these situations are discussed in more detail in Rogawski's lectures [Ro2].

1) Adjoint representation for $G L_{2}$. This is an instance where Example 2 can be carried out. For automorphic representations $\pi$ of $G L_{2}$, we consider $L$ functions $L(s, \pi, \mathrm{Ad})$, where $\mathrm{Ad}: G L_{2}(\mathbb{C}) \rightarrow G L_{3}(\mathbb{C})$ is the adjoint representation. As in Example 2, the goal is to exhibit these $L$ functions as standard $L$ functions $L(s, \Pi)$ with $\Pi$ automorphic for $G L_{3}$. This amounts to establishing global functoriality when $G=G L_{3}, H=G L_{2}$, and $\psi:{ }^{L} H \rightarrow{ }^{L} G$ is given by $\psi(x, \gamma)=(\operatorname{Ad}(x), \gamma)$. The positive result here is due to Gelbart and Jacquet [Gelb-Ja]. 
2) Base change for $G L_{2}$. Let $E$ be any finite extension of the number field $F$. Let $H=G L_{2}$ over $F$, and let $G=R_{E / F}\left(G L_{2}\right)$ be the group over $F$ given by restriction of ground field as in (6.5). As with (6.5), for any $F$ algebra $A$, $\left(R_{E / F}\right)\left(G L_{2}(A)\right)=G L_{2}\left(E \otimes_{F} A\right)$. Thus

$$
G(\bar{F})=G L_{2}\left(E \otimes_{F} \bar{F}\right)=G L_{2}(\bar{F}) \times \cdots \times G L_{2}(\bar{F})
$$

and

$$
G(F)=G L_{2}\left(E \otimes_{F} F\right)=G L_{2}(E) .
$$

The $L$ groups are

and

$$
{ }^{L} H=G L_{2}(\mathbb{C}) \times \operatorname{Gal}(\bar{F} / F)
$$

$$
{ }^{L} G=\left(G L_{2}(\mathbb{C}) \times \cdots \times G L_{2}(\mathbb{C})\right) \rtimes \operatorname{Gal}(\bar{F} / F),
$$

the second one being a semidirect product. The action of $\operatorname{Gal}(\bar{F} / F)$ on the product $G L_{2}(\mathbb{C}) \times \cdots \times G L_{2}(\mathbb{C})$ permutes the coordinates. This action factors through $\operatorname{Gal}(E / F)$ if $E$ is Galois over $F$. The map $\psi:{ }^{L} H \rightarrow{ }^{L} G$ is given by the diagonal map on the identity component and by the identity map on the Galois group. Langlands [Lgl5] proved global functoriality in this setting when $E / F$ is a cyclic Galois extension of prime degree; this is the long step in the proof of new cases of Artin's Conjecture established by Langlands. Arthur and Clozel [Ar-Cl] proved the corresponding instance of global functoriality for $G L_{n}$ when $E / F$ is cyclic Galois of prime degree.

There is also a considerable amount of more recent progress. Various newer results on the analytic properties of $L(s, \pi, r)$ are summarized in [Ra]. Rogawski [Ro1] has made an extensive study of automorphic representations of the group $U_{3}$. Here $E / F$ is a quadratic extension of number fields, and $G=U_{3}$ is the associated unitary group. For the group $H=U_{2} \times U_{1}$, there is an embedding ${ }^{L} H \rightarrow{ }^{L} G$, and Rogawski's work addresses functoriality for this map. Rogawski also studies functoriality for the map ${ }^{L} G \rightarrow{ }^{L} \widetilde{G}$, where $\widetilde{G}=R_{E / F}(G)$; this is the base change lifting from $U_{3}$ to $G L_{3}$ over $E$. This work is applied to arithmetic geometry in [Lgl$\mathrm{Ra}$; the forward of [Lgl-Ra] puts a number of aspects of the Langlands program in perspective.

\section{References}

[Ad-Ba-V] Adams, J., D. Barbasch, and D. A. Vogan, The Langlands Classification and Irreducible Characters for Real Reductive Groups, Birkhäuser, Boston, 1992.

[Ar-Cl] Arthur, J., and L. Clozel, Simple Algebras, Base Change, and the Advanced Theory of the Trace Formula, Princeton University Press, Princeton, 1989.

[Ar-Ta] Artin, E., and J. Tate, Class Field Theory, W. A. Benjamin Inc., New York, 1967.

[Ba-R] Barthel, L., and D. Ramakrishnan, A non-vanishing result for twists of $L$-functions of GL(n), Duke Math. J. 74 (1994), 681-700.

[Bl-Ro] Blasius, D., and J. D. Rogawski, Zeta functions of Shimura varieties, Motives, Proc. Symp. Pure Math., vol. 55, Part II, American Mathematical Society, Providence, 1994, pp. 525-571.

[Bo1] Borel, A., Some finiteness properties of adele groups over number fields, Publ. Math. I.H.E.S. 16 (1963), 5-30.

[Bo2] Borel, A., Linear algebraic groups, Algebraic Groups and Discontinuous Subgroups, Proc. Symp. Pure Math., vol. 9, American Mathematical Society, Providence, 1966, pp. 3-19.

[Bo3] Borel, A., Linear Algebraic Groups, W. A. Benjamin, New York, 1969. 
[Bo4] Borel, A., Automorphic L-functions, Automorphic Forms, Representations, and LFunctions, Proc. Symp. Pure Math., vol. 33, Part II, American Mathematical Society, Providence, 1979, pp. 27-61.

[Bo-HC] Borel, A., and Harish-Chandra, Arithmetic subgroups of algebraic groups, Annals of Math. 75 (1962), 485-535.

[Bo-Ja] Borel, A., and H. Jacquet, Automorphic forms and automorphic representations, Automorphic Forms, Representations, and L-Functions, Proc. Symp. Pure Math., vol. 33, Part I, American Mathematical Society, Providence, 1979, pp. 189-202.

[Buhl] Buhler, J. P., Icosahedral Galois Representations, Lecture Notes in Mathematics, vol. 654, Springer-Verlag, Berlin, 1978.

[Bump] Bump, D., Automorphic Forms and Representations, Cambridge University Press, Cambridge, 1997.

[Car] Cartier, P., Representations of p-adic groups: A survey, Automorphic Forms, Representations, and L-Functions, Proc. Symp. Pure Math., vol. 33, Part I, American Mathematical Society, Providence, 1979, pp. 111-155.

[Cas] Cassels, J. W. S., Global fields, Algebraic Number Theory (J. W. S. Cassels and A. Fröhlich, eds.), Academic Press, London, 1967, pp. 42-84.

[Cas-Fr] Cassels, J. W. S., and A. Fröhlich, Algebraic Number Theory, Academic Press, London, 1967.

[Ch] Chevalley, C., La théorie du corps de classes, Annals of Math. 41 (1940), 394-418.

[Co-Pi] Cogdell, J. W., and I. I. Piatetski-Shapiro, Converse theorems for $\mathrm{GL}_{n}$, Publ. Math. I.H.E.S. 79 (1994), 157-214.

[De-S] Deligne, P., and J.-P. Serre, Formes modulaires de poids 1, Annales Scient. Ecole Norm. Sup. 7 (1974), 507-530.

[Fl] Flath, D., Decomposition of representations into tensor products, Automorphic Forms, Representations, and L-Functions, Proc. Symp. Pure Math., vol. 33, Part I, American Mathematical Society, Providence, 1979, pp. 179-183.

[Fre] Frey, G. (ed.), On Artin's Conjecture for Odd 2-dimensional Representations, Lecture Notes in Mathematics, vol. 1585, Springer-Verlag, Berlin, 1994.

[Frö] Fröhlich, A., Local fields, Algebraic Number Theory (J. W. S. Cassels and A. Fröhlich, eds.), Academic Press, London, 1967, pp. 1-41.

[Ga] Garrett, P. B., Holomorphic Hilbert Modular Forms, Wadsworth \& Brooks/Cole, Pacific Grove, CA, 1990.

[Gelb1] Gelbart, S. S., Automorphic Forms on Adele Groups, Princeton University Press, Princeton, NJ, 1975.

[Gelb2] Gelbart, S. S., An elementary introduction to the Langlands program, Bull. Amer. Math. Soc. 10 (1984), 177-219.

[Gelb-Ja] Gelbart, S. S., and H. Jacquet, A relation between automorphic representations of GL(2) and GL(3), Annales Scient. École Norm. Sup. 11 (1978), 471-542.

[Gelf-Pi] Gelfand, I. M., and I. Piatetski-Shapiro, Automorphic forms and representation theory, Trudy Moscov. Mat. Obšč. 12 (1963), 389-412.

[Gf-Gr-P] Gelfand, I. M., M. I. Graev, and I. I. Piatetskii-Shapiro, Representation Theory and Automorphic Functions, W. B. Saunders, Philadelphia, 1969.

[Go-Ja] Godement, R., and H. Jacquet, Zeta-functions of simple algebras, Lecture Notes in Mathematics, vol. 260, Springer-Verlag, New York, 1972.

[Ha] Harris, M., Supercuspidal representations in the cohomology of Drinfel'd upper half spaces; elaboration of Carayol's program, preprint, 1994; Invent. Math. (to appear).

[HC1] Harish-Chandra, Automorphic forms on a semisimple Lie group, Proc. Nat. Acad. Sci. USA 45 (1959), 570-573.

[HC2] Harish-Chandra, Automorphic Forms on Semisimple Lie Groups, Lecture Notes in Mathematics, vol. 62, Springer-Verlag, Berlin, 1968.

[Hei] Heilbronn, H., Zeta-functions and L-functions, Algebraic Number Theory (J. W. S. Cassels and A. Fröhlich, eds.), Academic Press, London, 1967, pp. 204-230.

[Hen1] Henniart, G., La conjecture de Langlands locale pour GL(3), Mem. Soc. Math. France 11-12 (1984), 1-186.

[Hen2] Henniart, G., Caractérisation de la correspondence de Langlands locale par les facteurs $\varepsilon$ de paires, Invent. Math. 113 (1993), 339-350. 
[Ja1] Jacquet, H., Principal $L$ functions of the linear group, Automorphic Forms, Representations, and L-Functions, Proc. Symp. Pure Math., vol. 33, Part II, American Mathematical Society, Providence, 1979, pp. 63-86.

[Ja2] Jacquet, H., Principal $L$-functions for $G L(n)$, these Proceedings, pp. 321-329.

[Ja-Lgl] Jacquet, H., and R. P. Langlands, Automorphic Forms on GL(2), Lecture Notes in Mathematics, vol. 114, Springer-Verlag, Berlin, 1970.

[Ja-P-S] Jacquet, H., I. I. Piatetski-Shapiro, and J. A. Shalika, Rankin-Selberg convolutions, Amer. J. Math. 105 (1983), 367-464.

[Ja-Sha1] Jacquet, H., and J. A. Shalika, On Euler products and the classification of automorphic representations I, Amer. J. Math. 103 (1981), 499-558.

[Ja-Sha2] Jacquet, H., and J. Shalika, Rankin-Selberg convolutions: Archimedean theory, Festschrift in Honor of I. I. Piatetski-Shapiro on the Occasion of His Sixtieth Birthday, Part I (Ramat-Aviv, 1989), Israel Math. Conf. Proc., vol. 2, Weizmann, Jerusalem, 1990, pp. 125-207.

[Kna1] Knapp, A. W., Elliptic Curves, Princeton University Press, Princeton, 1992.

[Kna2] Knapp, A. W., Local Langlands correspondence: the archimedean case, Motives, Proc. Symp. Pure Math., vol. 55, Part II, American Mathematical Society, Providence, 1994, pp. 393-410.

[Kna-Vo] Knapp, A. W., and D. A. Vogan, Cohomological Induction and Unitary Representations, Princeton University Press, Princeton, NJ, 1995.

[Kne] Kneser, M., Strong approximation, Algebraic Groups and Discontinuous Subgroups, Proc. Symp. Pure Math., vol. 9, American Mathematical Society, Providence, 1966, pp. 187-196.

[Kud] Kudla, S. S., The local Langlands correspondence: the non-archimedean case, Motives, Proc. Symp. Pure Math., vol. 55, Part II, American Mathematical Society, Providence, 1994, pp. 365-391.

[Kut] Kutzko, P., The local Langlands conjecture for GL(2) of a local field, Annals of Math. 112 (1980), 381-412.

[La] Lang, S., Algebraic Number Theory, Springer-Verlag, New York, 1986.

[Lgl1] Langlands, R. P., Representations of abelian algebraic groups, duplicated notes, Yale University, New Haven, CT, 1968.

[Lgl2] Langlands, R. P., Problems in the theory of automorphic forms, Lectures in Modern Analysis and Applications III, Lecture Notes in Mathematics, vol. 170, SpringerVerlag, Berlin, 1970, pp. 18-61.

[Lgl3] Langlands, R. P., On the classification of irreducible representations of real algebraic groups, mimeographed notes, Institute for Advanced Study, Princeton, NJ, 1973, Representation Theory and Harmonic Analysis on Semisimple Lie Groups (P. J. Sally and D. A. Vogan, eds.), Math. Surveys and Monographs, vol. 31, American Mathematical Society, Providence, 1989, pp. 101-170.

[Lgl4] Langlands, R. P., On the notion of an automorphic representation, Automorphic Forms, Representations, and L-Functions, Proc. Symp. Pure Math., vol. 33, Part I, American Mathematical Society, Providence, 1979, pp. 203-207.

[Lg15] Langlands, R. P., Base Change for GL(2), Princeton University Press, Princeton, 1980.

[Lgl6] Langlands, R. P., Representation theory: its rise and its role in number theory, Proceedings of the Gibbs Symposium (New Haven, CT, 1989), American Mathematical Society, Providence, RI, 1990, pp. 181-210.

[Lgl7] Langlands, R. P., Where stands functoriality today?, these ProceEdings, pp. 457-471.

[Lgl-Ra] Langlands, R. P., and D. Ramakrishnan (eds.), The Zeta Functions of Picard Modular Surfaces, Les Publications CRM, Montréal, 1992.

[Maa] Maass, H., Über eine neue Art von nicht analytischen automorphen Funktionen und die Bestimmung Dirichletscher Reihen durch Funktionalgleichungen, Math. Annalen 121 (1949), 141-183.

[Mar] Martinet, J., Character theory and Artin L-functions, Algebraic Number Fields: L-Functions and Galois Properties (A. Fröhlich, ed.), Academic Press, London, 1977, pp. $1-87$.

[Moe1] Mœglin, C., Representations of GL(n) over the real field, these ProceEdings, pp. $157-166$. 
[Moe2] Møglin, C., Representations of GL(n,F) in the nonarchimedean case, these ProceEdINGS, pp. 303-319.

[Moy] Moy, A., Local constants and the tame Langlands correspondence, Amer. J. Math. 108 (1986), 863-929.

[Ne] Neukirch, J., Class Field Theory, Springer-Verlag, Berlin, 1986.

[Pi] Piatetski-Shapiro, I., Festschrift in Honor of I. I. Piatetski-Shapiro on the Occasion of His Sixtieth Birthday, Part II, Israel Math. Conf. Proc., vol. 3, 1990, pp. 185-195.

[Ra] Ramakrishnan, D., Pure motives and automorphic forms, Motives, Proc. Symp. Pure Math., vol. 55, Part II, American Mathematical Society, Providence, 1994, pp. 411-446.

[Ro1] Rogawski, J. D., Automorphic Representations of Unitary Groups in Three Variables, Princeton University Press, Princeton, 1990.

[Ro2] Rogawski, J. D., Functoriality and the Artin conjecture, these Proceedings, pp. 331-353.

[Se1] Serre, J.-P., Local class field theory, Algebraic Number Theory (J. W. S. Cassels and A. Fröhlich, eds.), Academic Press, London, 1967, pp. 129-161.

[Se2] Serre, J.-P., Local Fields, Springer-Verlag, New York, 1979.

[Sha] Shalika, J. A., The multiplicity one theorem for $\mathrm{GL}_{n}$, Annals of Math. 100 (1974), 171-193.

[Shi] Shimura, G., Introduction to the Arithmetic Theory of Automorphic Functions, Princeton University Press, Princeton, NJ, 1971.

[Si] Siegel, C. L., Topics in Complex Function Theory, vol. III, Wiley-Interscience, New York, 1973.

[Sp] Springer, T. A., Reductive groups, Automorphic Forms, Representations, and LFunctions, Proc. Symp. Pure Math., vol. 33, Part I, American Mathematical Society, Providence, 1979, pp. 3-27.

[Ta1] Tate, J. T., Fourier analysis in number fields and Hecke's zeta-functions, Ph.D. Thesis, Princeton University, 1950, Algebraic Number Theory (J. W. S. Cassels and A. Fröhlich, eds.), Academic Press, London, 1967, pp. 305-347.

[Ta2] Tate, J. T., Global class field theory, Algebraic Number Theory (J. W. S. Cassels and A. Fröhlich, eds.), Academic Press, London, 1967, pp. 162-203.

[Ta3] Tate, J., Number theoretic background, Automorphic Forms, Representations, and LFunctions, Proc. Symp. Pure Math., vol. 33, Part II, American Mathematical Society, Providence, 1979, pp. 3-26.

[Ti] Tits, J., Reductive groups over local fields, Automorphic Forms, Representations, and L-Functions, Proc. Symp. Pure Math., vol. 33, Part I, American Mathematical Society, Providence, 1979, pp. 29-69.

[Tu] Tunnell, J., On the local Langlands conjecture for GL(2), Invent. Math. 46 (1978), 179-200.

[We1] Weil, A., Adeles and Algebraic Groups, bound notes, Institute for Advanced Study, Princeton, NJ, 1961.

[We2] Weil, A., Über die Bestimmung Dirichletscher Reihen durch Funktionalgleichungen, Math. Annalen 168 (1967), 149-156.

[We3] Weil, A., Dirichlet Series and Automorphic Forms, Lecture Notes in Mathematics, vol. 189, Springer-Verlag, Berlin, 1971.

[We4] Weil, A., Basic Number Theory, Springer-Verlag, New York, 1973.

Department of Mathematics, State University of New York, Stony Brook, New York 11794, U.S.A.

E-mail address: aknapp@ccmail.sunysb.edu 